University of Nebraska - Lincoln

DigitalCommons@University of Nebraska - Lincoln

\title{
Interaction of laser radiation with a negative ion in the presence of a strong static electric field
}

\author{
N. L. Manakov \\ Voronezh State University, manakov@phys.vsu.ru \\ M. V. Frolov \\ Voronezh State University, Russia, frolov@phys.vsu.ru \\ Anthony F. Starace \\ University of Nebraska-Lincoln, astarace1@unl.edu \\ Ilya I. Fabrikant \\ University of Nebraska-Lincoln, ifabrikant@unl.edu
}

Follow this and additional works at: https://digitalcommons.unl.edu/physicsstarace

Part of the Physics Commons

Manakov, N. L.; Frolov, M. V.; Starace, Anthony F.; and Fabrikant, Ilya I., "Interaction of laser radiation with a negative ion in the presence of a strong static electric field" (2000). Anthony F. Starace Publications. 176. https://digitalcommons.unl.edu/physicsstarace/176

This Article is brought to you for free and open access by the Research Papers in Physics and Astronomy at DigitalCommons@University of Nebraska - Lincoln. It has been accepted for inclusion in Anthony F. Starace Publications by an authorized administrator of DigitalCommons@University of Nebraska - Lincoln. 
TOPICAL REVIEW

\title{
Interaction of laser radiation with a negative ion in the presence of a strong static electric field
}

\author{
N L Manakov †, M V Frolov $\dagger$, A F Starace $\ddagger$ and I I Fabrikant $\ddagger$ \\ $\dagger$ Department of Physics, Voronezh State University, Voronezh 394693, Russia \\ ¥Department of Physics and Astronomy, The University of Nebraska, Lincoln, NE 68588-0111, \\ USA
}

Received 23 February 2000

\begin{abstract}
This paper provides a general theoretical description of a weakly bound atomic system (a negative ion) interacting simultaneously with two (generally strong) fields, a static electric field and a monochromatic laser field having an arbitrary elliptical polarization. The zerorange $\delta$-potential is used to model the interaction of a bound electron in a negative ion as well as the interaction of a detached electron with the residual atom. Our treatment combines the quasistationary (complex energy) and quasienergy (Floquet) approaches. This quasistationary, quasienergy state (QQES) formalism is the most appropriate one for analysing a decaying quantum system under the influence of a periodic external perturbation. Existing QQES theory is reviewed and some new results are discussed: the Hellmann-Feynman theorem and the normalization procedure for QQES, and the definition of the dipole moment and the dynamic polarizability for a decaying atomic system (in strong static electric and/or laser fields). These results are illustrated using analytical formulae obtained from an exact solution of the QQES problem for a $\delta$-model potential in two strong fields. Finally, from the imaginary part of the dynamic polarizability we obtain analytic results (to first order in the laser-field intensity) for the photodetachment cross section. Our results are then compared with those of previous theoretical studies.
\end{abstract}

\section{Contents}

Contents

1. Introduction

1.1. A brief historical survey of static electric field effects on photodetachment processes

1.2. A brief historical survey of the quasienergy and quasistationary, quasienergy approaches

1.3. Outline of the contents of this review 150

2. Basic equations of the QQES approach $\quad 152$

3. Solution of the QQES problem for the zero-range potential in two fields 155

3.1. Geometry of the fields and definitions of scaled units 156

3.2. Exact results for $\epsilon$ and $\Phi_{\epsilon}(\boldsymbol{r}, t)$ in scaled units $\quad 157$

3.3. Some special cases $\quad 160$

4. Complex energy of QS in a static electric field 161

5. Weak-laser-field expansion of the exact result for $\epsilon \quad 163$

0953-4075/00/150141+74\$30.00（C) 2000 IOP Publishing Ltd R141 
6. The normalization procedure for QQES and PT calculation of $\Delta \epsilon^{\mathrm{opt}}$ in the basis of quasistationary states

6.1. Linear in $F$ perturbative expansion of $\Phi_{\epsilon}(r, t)$

6.2. Normalization of the QQES wavefunctions 167

6.3. Perturbative calculation of $\Delta \epsilon^{\text {opt }}$ with quasistationary basis functions 171

7. The dipole moment and polarizability of a decaying atomic system 172

7.1. Connection between the polarizability and the dynamical Stark effect for a weak laser and zero static field

7.2. The dual dipole moment and the nonlinear static polarizability in a strong static electric field

7.3. The dual dipole moment and the dynamic polarizability for weak laser and strong static fields

7.4. Static-electric-field-induced polarization anomalies in light scattering by atoms and ions

$\begin{array}{ll}\text { 7.5. Summary and observations } & 178\end{array}$

8. Analysis of the dynamic polarizability $\alpha(\mathcal{F} ; \omega) \quad 180$

8.1. Below- and above-threshold asymptotics 183

8.2. Behaviour of $\alpha(\mathcal{F} ; \omega)$ near and at threshold $\quad 185$

9. The photodetachment cross section and connections with previous studies $\quad 190$

9.1. General considerations $\quad 190$

9.2. Analysis of alternative approximations 193

9.3. Discussion 200

10. Conclusions 203

Acknowledgments 204

Appendix A. Green functions for a free electron in static electric and laser fields 204

Appendix B. Calculation and regularization of some integrals 206

Appendix C. Limiting expressions for the regularized Green function $g_{\mathcal{F}}(E)$ and its

derivatives 208

$\begin{array}{ll}\text { References } & 209\end{array}$

\section{Introduction}

Few problems in physics have analytical solutions, particularly those that are time dependent. Dodonov and Man'ko [1] and Kleber [2] have enumerated those time-dependent problems which do have analytic solutions, and have provided analytic, time-dependent propagators for them. However, few of the problems analysed are relevant to currently possible and interesting experiments. Of those currently interesting physical problems, those for which atomic interactions may be modelled by a zero-range ( $\delta$-function) potential [3] permit the most complete theoretical analysis. (See also the rather mathematical treatments for this potential in [4].) In this review we consider one such problem: laser detachment of an electron bound by a short-range, $\delta$-model potential in the presence of a strong static electric field. (Note that the bound solution of a $\delta$-model potential provides an excellent representation for the $\mathrm{H}^{-}$negative ion, as well as others.) Though this problem has a long history, most prior treatments have involved significant approximations or else have been restricted to low static field values, particular field geometries, and/or particular cases of laser polarization. Other treatments include only rather general equations and do not provide analytical or numerical results. For all of these reasons, it is difficult to relate one prior treatment with another, as there is no exact result with which to compare. This problem of interpretation is particularly acute for the case of strong static electric fields because nearly all prior 
treatments assume the initial state to be unaffected by the fields involved, whereas one knows that the very notion of a bound state in a static field (as well as in a strong laser field) is erroneous.

In this review we formulate an analytic solution to the problem of laser detachment of an electron bound in a $\delta$-model potential in the presence of a strong static electric field. We begin our analysis using a quasienergy approach [5], or, more precisely, a quasistationary quasienergy approach [6] to account for the decay caused by a strong laser and/or static field. Our analytic results for the complex quasienergy are valid for any values of the static or laser-field strengths. Using those results we then analyse conditions under which one may define a dynamical polarizability and, from its imaginary part, a photodetachment cross section. In order to compare with prior work we present detailed analytical formulae for the photodetachment cross section that are correct to first order in the laser-field amplitude. These formulae allow us to relate prior work by others for weak laser fields to the exact treatment we present.

Differences between the results of prior work and the exact results presented here are greatest for strong static fields. Such differences are present and significant for static field values comparable to those which have been achieved experimentally and for which it remains possible to define a photodetachment cross section. Static electric field strengths of up to $3.5 \mathrm{MV} \mathrm{cm} \mathrm{cm}^{-1}$ have been achieved in experiments using a relativistic $\mathrm{H}$ atom beam to convert a modest laboratory magnetic field into a static electric field in the atom rest frame $[7,8]$. Similar experiments for the $\mathrm{H}^{-}$ion have employed rest-frame static electric fields of $1.3 \mathrm{MV} \mathrm{cm}^{-1}$ and higher to examine the effect of such fields on the well known photodetachment resonance structures near the $n=2$ threshold [8,9]. For static fields above $2 \mathrm{MV} \mathrm{cm}^{-1}$ the process of field ionization becomes increasingly important [9]. Nevertheless, the essential point is that, first, experiments for photodetachment of $\mathrm{H}^{-}$in static electric fields of the order of $1 \mathrm{MV} \mathrm{cm}^{-1}$ are capable of being done, and, second, that such experiments would clearly differentiate among the various theoretical predictions which are analysed in this review. Similar experiments for negative ions having higher electron affinities than $\mathrm{H}^{-}$ would furthermore permit photodetachment studies in even higher static fields.

\subsection{A brief historical survey of static electric field effects on photodetachment processes}

The effect of an external electric field on the optical-absorption properties of excitons, insulators and semiconductors attracted much theoretical interest quite some time ago [10-21]. In the 1970s, this theoretical interest also extended to the effect of external electric fields on multiphoton detachment processes [22-25]. For single-photon absorption both by solids and by negative ions, there were explicit theoretical predictions of oscillatory behaviour above the absorption thresholds [12,24]. Arutyunyan and Askar'yan [22] have given, as far as we know, the first qualitative overview of the general process of multiphoton detachment in the presence of a static uniform electric field. They focused on the dependence of the detachment process on the laser frequency and on the field intensities. Nikishov [23] gave a general formal solution for the multiphoton transition rates using a gauge in which both fields are described by a vector potential. Nikishov [23] presents detailed results of the influence of the static field on the multiphoton detachment process, however, for only two simple cases: the case of laser photons that are linearly polarized and the case of the low-frequency limit. Slonim and Dalidchik [24] have treated both single-photon and multiphoton detachment of negative ions in a static uniform electric field. For single-photon detachment, they treated the coupling of the negative ion to the electromagnetic field perturbatively for two cases: that of collinear static electric and linearly polarized electromagnetic fields and the case of an unpolarized light beam 
collinear to the direction of a static field, i.e. an orthogonal geometry. For the multiphoton case, only the simplest instance, when a circularly polarized laser beam is collinear to a static electric field vector, was considered and treated non-perturbatively. This case of orthogonal geometry does not involve oscillations of the multiphoton cross sections. However, Slonim and Dalidchik did show that even for this case the static field could increase the ratio of the three-photon detachment cross section to the two-photon detachment cross section. Manakov and Fainshtein [25] presented general equations for the decay of a weakly bound electron in both a static uniform electric field and a strong electromagnetic field. A detailed analysis for the case of circular polarization and orthogonal geometry showed that the presence of the electromagnetic field could increase the rate of decay by tunnelling by several orders of magnitude.

The experimental observations of the oscillatory resonance behaviour in the photoionization spectra of $\mathrm{Rb}$ both above the classical ionization threshold [26] and especially above the zero-field-ionization threshold [27] stimulated detailed theoretical investigations of atomic photoionization [28-35] as well as photodetachment $[31,34,36]$ in the presence of a weak static electric field. Subsequent experimental observations [37] of electric-fieldinduced resonances in the photodetachment spectrum of $\mathrm{H}^{-}$induced a resurgence of activity on primarily single-photon detachment processes in the presence of a weak external electric field [38-46]. More recently, theorists have returned to the investigation of static-field effects on single-photon and multiphoton detachment processes (for the case in which the electric field can no longer be regarded as a weak perturbation) in order to provide detailed predictions which experiment may be capable of testing [47-52]. In what follows, we discuss briefly some of these works.

The theoretical work since 1980 on photodetachment of negative ions in the presence of a static electric field has primarily addressed the interesting case of collinear laser polarization and static electric field directions, for which photodetachment cross sections display oscillations. Nearly all of this theoretical work has involved significant approximations, which are appropriate for weak static fields but which become increasingly less valid the stronger the static field. Fabrikant [31] used classical and semiclassical analyses to interpret the known cross section oscillations in terms of interference between two classical trajectories for the electron detached from a negative ion. In the direct detachment trajectory, the electron moves along the direction of decreasing potential energy and is accelerated away from the atom by the static field. In the other, indirect detachment trajectory, the electron moves initially in the direction of increasing potential energy, is reflected by the static field at the classical turning point, and then is accelerated away from the atom. The extent of interference between the direct and the indirect detachment trajectories is sensitive to the frequency of the light which detaches the initially bound electron, thereby resulting in an oscillatory structure in the photodetachment cross section as a function of light frequency. Fabrikant did not provide any numerical results showing the magnitude of these oscillations.

Those works which did provide numerical predictions generally ignored final-state interactions of the detached electron with the atom (i.e. a 'static field Born approximation') and also made other approximations in evaluating the spatial integrals involved in the relevant matrix elements. Thus, Reinhardt and Overman in [37] obtained the photodetachment cross section from the dipole autocorrelation function [36], which was evaluated analytically using an approximate analytic form for the detached electron wavefunction. Rau and Wong [38] and Greene and Rouze [40] used a frame transformation approach to connect the wavefunctions at large distances describing electron motion in a static electric field with the wavefunction at small radial distances, where it was assumed that the static field could be ignored. Du and Delos [41] evaluated the electric dipole radial matrix element using a stationary phase 
approximation. Kondratovich and Ostrovsky [43] summed over final-state partial waves using a Watson transformation and a pole approximation.

More detailed theoretical analyses have sought to include effects of final-state electronatom interactions. If the detached electron has an orbital angular momentum $l>0$ and if the static electric field is weak (so that angular momentum mixing effects are small), then such final-state interaction effects are expected to be negligible. However, for strong static electric fields and especially for cases when the detached electron has $l=0$, such final-state interaction effects are expected to be significant. From a classical point of view, the indirect electron trajectory (in which the electron is reflected by the static field back to the atom) provides an opportunity for the electron to be scattered by the atom. (Given that this may happen repeatedly, with the electron wavepacket oscillating between the atom and the classical turning point in the static field, one speaks of the process more generally as the 'rescattering effect'.) Fabrikant [34] formulated a multichannel photodetachment theory to describe such effects, but no numerical results were presented. Wong et al [39] employed a frame transformation approach together with a multichannel quantum defect theory description of the final-state wavefunction in the vicinity of the atom in order to provide a detailed treatment of $\mathrm{S}^{-}$photodetachment in a weak static field.

Subsequent theoretical work has sought to provide a more precise description of final-state interaction effects appropriate even for strong static electric fields. Nicolaides and Mercouris [47] treated all final-state interactions in principle exactly (but completely numerically) for the case of photodetachment of $\mathrm{Li}^{-}$and $\mathrm{H}^{-}$. However, for weak fields their results only confirmed results of simpler theoretical calculations as well as the experimental measurements for $\mathrm{H}^{-}$ photodetachment [37]. No new effects were predicted for stronger fields. Fabrikant [48] used a frame transformation technique to treat the final-state rescattering of the detached electron by the residual atom for the case of a weak laser field. He found that the rescattering effect significantly lowers the photodetachment cross section of $\mathrm{H}^{-}$for strong static electric fields near the zero-static field threshold (relative to predictions of those calculations which ignore final-state rescattering effects). Attempts were also made [49] to include the rescattering effect in a theoretical treatment of photodetachment of complex ions, $\mathrm{S}^{-}$and $\mathrm{Cl}^{-}$, in order to describe the experiments [50] for these ions in moderate fields of $1 \mathrm{kV} \mathrm{cm}^{-1}$. Ostrovsky and Telnov $[45,46]$ have carried out an analytic study of the photodetachment of negative ions that, in principle, includes all final-state interaction effects: the case of linear polarization of the laser field was analysed in [45] and that of an elliptical polarization in [46]. Their focus is on the particular case of a strong laser field and a weak static electric field. However, no numerical results were provided.

Gao and Starace [51] were the first to treat the multiphoton and single-photon detachment in the presence of a static field with the exact inclusion of the field effects. Their result for single-photon detachment contains an extra term which is important for strong static fields. Bao et al [52] developed an approach combining the strong field effects of Gao and Starace [51] and the final-state rescattering effect of Fabrikant [49]. Their numerical results are close to the exact results presented in the present paper.

A few photodetachment studies are related tangentially to the subject of this paper. Slonim and Greene [53] used a frame transformation technique and multichannel quantum defect theory to treat final-state rescattering effects on the photodetachment of $\mathrm{H}^{-}$, while ignoring the final-state interaction of the electron with the laser field. The focus of their study is on the effect of a static field on the well known shape and Feshbach resonances near the $\mathrm{H}(n=2)$ threshold rather than on the photodetachment cross section near the $\mathrm{H}(n=1)$ threshold. Du et al [54] also used a frame transformation approach but one based on ab initio numerical adiabatic hyperspherical transition amplitudes (which include 
final-state electron-atom interaction effects) to study static electric field effects on the shape and Feshbach resonances near the $\mathrm{H}(n=2)$ threshold in $\mathrm{H}^{-}$photodetachment. They also ignored the final-state interaction of the electron with the laser field and did not provide predictions for the $\mathrm{H}(n=1)$ photodetachment cross section. Delone et al [55] noticed that oscillations similar to those induced by a static field can be observed in a lowfrequency field (see also [56]). Finally, an interesting static-field-induced-effect is the spatial oscillation of the flux of photodetached electrons. This oscillation was first predicted by Fabrikant [31]. Demkov et al [57] proposed an experiment in which this effect can be observed and called it the 'photoionization microscope' since it allows a direct measurement of the electron wavefunction. Recently this experiment was realized by Blondel et al [58].

\subsection{A brief historical survey of the quasienergy and quasistationary, quasienergy approaches}

The concepts of quasienergy and of quasienergy states (QES) for a system in a time-periodic external field were introduced in the mid-1960s $[5,59,60]$ and at present many problems involving strong laser-field interactions with atomic and molecular systems are investigated using QES (or Floquet states). Based on the Floquet theorem [61], Shirley [59] reduced the solution of the time-dependent Schrödinger equation for a finite (two-)level quantum system in an oscillating field to a time-independent, infinite Floquet matrix. The term quasienergy for the Floquet index $\epsilon$, i.e. for a new, conserved quantum number of a quantum system in a time-periodic perturbative potential, was introduced by Zel'dovich [5] and by Ritus [60] in analogy with the conserved quasimomentum of an electron in a spatially periodic crystal field (which defines the Bloch states). Ritus [60] employed QES to analyse the linear in laser intensity light shift of hydrogen atom levels. A major contribution to the QES approach was made by Zel'dovich [5, 62], who analysed the basic properties of QES and argued that this method presents the most convenient and natural way to describe a quantum system subjected to a periodic external perturbation which is treated semiclassically. The next major step in the formal analysis of QES was made by Sambe [63]. He introduced an enlarged Hilbert space as the direct product, $R_{3} \oplus T$, of the configuration Hilbert space, $R_{3}$, and the complete orthonormal set, $T$, of functions $\exp (\mathrm{i} k \omega t)$, where $k=0, \pm 1, \pm 2, \ldots$ In this representation, the Schrödinger equation for $\epsilon$ and for the time-periodic QES wavefunction $\Phi_{\epsilon}(\boldsymbol{r}, t)$ (see (4) and (5) below) in the space $R_{3} \oplus T$ is formally similar to the time-independent Schrödinger equation for a conservative system in $R_{3}$. Moreover, most of the basic theorems for timeindependent problems (e.g. the variational principle, the Hellmann-Feynman theorem and the perturbation theory (PT) formalism) are valid for the quasienergy eigenvalue problem in $R_{3} \oplus T$-space [63]. In [64] some other general properties of QES having a discrete spectrum of quasienergies were considered, in particular, the 'Brillouin zones' and Wigner-Neumann theorem on the crossing or avoided-crossing of quasienergy levels when $F$ and/or $\omega$ are varied, and the notion of the 'mean energy', $\overline{\mathcal{E}}$, for a time-periodic Hamiltonian. This latter quantity is closely related to the quasienergy: $\overline{\mathcal{E}}=\epsilon-\omega \partial \epsilon / \partial \omega$. It has proved useful, for instance, in molecular problems [65].

The number of exactly solvable eigenvalue problems involving the complete QES basis set is relatively small. Analytical solutions exist for any system whose Hamiltonian is quadratic in the momenta and coordinates (e.g. a free electron or a harmonic oscillator in a periodic field). Detailed analyses of such simple cases is given in the books [66,67]. Exact results for the quasienergy spectra of two- and three-dimensional rotators in a strong circularly polarized field are presented in $[64,68,69]$. The QES in the one-dimensional $\delta$-model potential with the depth depending harmonically on time have been examined in [70] (see also [71]). The quasienergy 
structure of degenerate hydrogen levels in a weak, low-frequency laser field was analysed analytically for circularly polarized [72] and for linearly polarized [73] laser fields. More general results, valid for an arbitrary frequency, were obtained in [74, 75], where the transition from the quadratic to the linear Stark shift was also predicted as the field amplitude increases for a fixed (infrared) frequency $\omega$. This interesting feature of the QES spectrum of hydrogen originates from the permanent dipole moment of excited H-levels. A specific behaviour of the quasienergy spectrum of hydrogen Rydberg states in an elliptically polarized laser and in a magnetic field was discussed recently in [76]. Concerning analyses of linear shifts in laser intensity of degenerate hydrogen atom levels see, e.g., [77, 78]. A rather intensive discussion of applications of results for the quasienergy structure of hydrogen atom levels, in particular, for plasma diagnostics and line broadening using time-dependent and static electric and/or magnetic fields, is presented in the book [79] (see also references therein). Many authors have also investigated a two-level system in an arbitrarily strong field using QES ideas (see, e.g., [59, 64, 69, 80, 81] and the review [82]). A number of applications of the Floquet matrix approach for finite (mainly two- and three-) level systems to molecular problems (Stark shifts, excitation, etc) together with different non-perturbative methods for numerical calculations for these problems have been reviewed in [83].

In a rigorous formulation of the problem of a real atomic system in a strong laser field, the quasienergy spectrum is purely continuous due to the possibility of decay (by ionization, dissociation, etc). Therefore, the QES approach in its original form $[5,59,60]$ is useful only for collisional problems. It provides the complete set of scattering states normalized to a $\delta$-function in the quasienergy. Such functions are convenient for analysing, for example, laser-assisted electron-atom collisions. Note that only two examples of an exact analytical solution for the three-dimensional case are known to us: electron scattering by a three-dimensional $\delta$-potential in the presence of a strong circularly polarized light wave [84] and electron scattering by a separable potential $[85,86]$. A rather extensive literature exists on the approximate analytical as well as numerical analyses of laser-assisted scattering using the QES approach. We note here only [87] and a more recent review [88]. Concerning non-perturbative Floquet methods for numerical calculations of electron scattering by complex atoms in a laser field see the review [89].

In the mid-1970s the QES approach developed originally for the Hermitian eigenvalue problem in the QES space, $R_{3} \oplus T$, was generalized to account for laser-field-induced widths of atomic levels, i.e. ionization rates in a strong field. (Note that in the earlier Sambe analyses [63] the contribution of the continuous spectrum of an unperturbed atomic system was not taken into account.) This generalization was realized by an extension of the well known theory of quasistationary (or resonance) states (QS) for time-independent Hamiltonians (see, e.g., $[66,90,91]$ as well as the pedagogical paper [92]) to the 'stationary QES Schrödinger equation' in $R_{3} \oplus T$. The term 'complex quasienergy' was introduced in a non-perturbative analysis of the decay of a weakly bound level (in a $\delta$-potential model) in a strong circularly polarized field [84,93] (see also [94]). In [93] the complex quasienergy, $\epsilon=\operatorname{Re} \epsilon-\frac{1}{2} \mathrm{i} \Gamma$, was obtained similarly to the standard quasistationary approach [90], i.e. as a complex eigenvalue of the QES Hamiltonian for the solution satisfying an outgoing wave boundary condition. Here $\operatorname{Re} \epsilon-E_{0}$ and $\Gamma$ give the Stark shift and width, i.e. the decay rate, of an initial bound state with energy $E_{0}$. The transcendental equation for $\epsilon$ obtained in [93] was also obtained in [84] using the resonance state formalism [91], i.e. as an equation for the pole of the scattering state QES solution in the complex $\epsilon$-plane. Note that a simplification of the QES analysis for the case of a circularly polarized field occurs owing to an obvious symmetry: transformation to the coordinate frame rotating with the frequency of the field removes the time dependence of the Hamiltonian [95]. The closed form of the time-evolution operator for this case was 
discussed in [96] and, with more mathematical details, in [97]. (See also [98] for more extensive numerical results for a $\delta$-model potential as compared with those in [84,93].) The simplicity of the circular polarization case allowed the generalization of the results in $[84,93]$ to more subtle cases: to states with non-zero angular momenta in a short-range potential [99], and to a quasimolecular negative ion modelled by two zero-range potentials [100]. The exact solution of the quasistationary, quasienergy state (QQES) problem for a zero-range potential in an elliptically polarized field was obtained in [25]. We also mention an alternative way $[101,102]$ to analyse the decay of a system in a laser field, based on the use of QES scattering states and a projection operator technique for separating the effects of the bound and continuum states.

Obviously, for real atomic potentials an analytic solution of the QQES problem is impossible, and hence approximate or numerical solutions or methods of analysis have been sought. The complex quasienergy of atoms in ground and excited states with a perturbative account of a laser field in higher-order PT was calculated numerically in [75] and analysed in terms of linear and nonlinear (hyper)polarizabilities, whose imaginary parts give the level width. An analysis of the position of the $S$-matrix poles on the Riemann surface for the complex quasienergy was performed in [103] (see also [104]), and the behaviour of the trajectories of poles when the parameters of the problem vary was demonstrated, based on the exactly solvable model [76]. A number of theoretical problems of the QQES approach were treated in $[105,106]$ (cf also section 2 below) based on the formal analysis of quasistationary states in the time-independent problem [91]. In particular, there are integral equations for QQES; a formal analysis for a level shift and width operator; perturbation theory in terms of a reduced, QES Green function of an atomic system; analyses of the convergence of PT series and of the exponential (in time) law for the decay of an atom in a strong monochromatic field. An adiabatic (low-frequency) approximation for the quasienergy shift and width was presented in [107]. An effective algorithm for a non-perturbative numerical calculation of the complex quasienergy based on methods of complex scaling (dilatation) [108] of the QQES Hamiltonian and $L^{2}$-continuum discretization [109] was suggested in [110] as well as demonstrated in calculations for the hydrogen atom (see [83] and the more thorough analysis of this technique in [111]). The most complete investigation of complex quasienergies for the hydrogen atom was performed in a number of papers by Shakeshaft, Potvliege and coauthors (see reviews $[112,113]$ and references therein). These authors also performed a detailed numerical analysis $[114,115]$ of the analytical structure of complex quasienergies of the $\mathrm{H}$ atom as a function of the amplitude, $F$, and frequency, $\omega$, of a laser field. This question is important, in particular, owing to the well known non-analyticity of the (complex) energy of an atomic electron in a static electric field $\mathcal{F}$ at $\mathcal{F}=0$. In contrast with the well known asymptotic property of the PT expansion for the energy in a static field, which is a real (asymptotic) expansion, in $[106,116]$ the convergence of the PT expansion (in the amplitude of the laser field) for the complex quasienergy was demonstrated. More detailed analyses and calculations were carried out for the $\mathrm{H}$ atom [114] and for the $\delta$-model potential [117]. For a rigorous mathematical treatment of the convergence properties of PT for complex quasienergies, see [118]. For complex atoms, the trajectories of complex quasienergies in the complex $\epsilon$-plane have a rich structure compared with the Coulomb case [119]. Since exact numerical calculations in the QQES (or non-Hermitian Floquet) approach are rather tedious and cumbersome, in the last decade the exact QQES solution for a $\delta$-model in an elliptically polarized field [25] was revived and has found wide application in model non-perturbative calculations of above-threshold ionization and high-harmonic generation by atoms in a strong laser field. An approximate version of this solution was used in a number of papers by Becker and co-authors (see, e.g., [120]). The results obtained confirm 
a number of predictions of a semiclassical three-step model [121] for strong laser-field-atom interactions.

Finally, in the mid-1980s the QES and QQES analyses developed originally for strictly monochromatic external perturbations, were generalized for the case of two- (or more) colour laser fields with incommensurable frequencies. In [122] such a generalization was performed for a finite-level system by an exact extension of Shirley's Floquet-matrix formalism for a twolevel (or spin- $\frac{1}{2}$ ) system in a bichromatic field with frequencies $\omega_{1}$ and $\omega_{2}$. This generalization allows one to treat the time-dependent problem of any finite-level system in a two-colour field as an equivalent time-independent, infinite-dimensional eigenvalue problem (see [83] for a number of examples). A more general extension of the quasienergy approach to the case of two-frequency fields was suggested in [55] based on an extension of the Sambe analysis [63] for the $R_{3} \oplus T$-space to the space $R_{3} \oplus T_{1} \oplus T_{2}$, where $T_{1,2}$ are the subspaces of periodic functions with frequencies $\omega_{1,2}$. In this method the 'generalized' (two-colour) quasienergy $\epsilon$ is defined as an eigenvalue of a 'two-time Schrödinger equation' [55] for the auxiliary function $\Phi_{\epsilon}\left(\boldsymbol{r}, t_{1}, t_{2}\right)$, which is periodic in $t_{1}$ and $t_{2}$, and which, for $t_{1}=t_{2}=t$, gives the QES solution of an initial two-colour QES problem. Note that, as a function of $t$, the two-colour QES function $\Phi_{\epsilon}(r, t, t)$ does not satisfy any periodic conditions, but nevertheless it is a particular solution (having a special form) for the original time-dependent Schrödinger equation and describes some stable, 'stationary' state of the quantum system in two fields, which is completely similar to the QES wavefunction for the one-colour field. From a mathematical point of view, the two-colour QES functions are related to so-called 'almost-periodic functions' [123], which have important applications in mathematical physics. All general theorems for ordinary QES can be generalized to the two-colour case, and solutions of the above-mentioned two-time eigenvalue equation with radiation (outgoing-wave) conditions at $r=|\boldsymbol{r}| \rightarrow \infty$ give us the two-colour QQES with a complex quasienergy whose imaginary part is the decay rate of a quantum system in two monochromatic fields (see the review [124] for more details). The mathematical treatment of an extension of the QES approach for polychromatic fields is presented in [125]. The first non-perturbative application of the QQES theory to two-colour problems was presented in [55] for the $\delta$-model potential in two strong fields, one infrared and the other optical. For this case, the one-dimensional integral equation for the complex quasienergy is similar to that for the one-colour case (see [25] or equation (29) below), differing only in the form of the Green function, which is that for a free electron in a two-colour laser field. In particular, the generalization of the well known Keldysh formula for tunnelling ionization [126] was obtained in [55] for the case of tunnelling (in a strong infrared field) not from the real bound state but from a harmonic of the QES in a (weak) optical field. Interest in the two-colour problem in strong fields has been stimulated over the past decade by experiments on above-threshold ionization and on high-harmonic generation with two-colour laser fields (see, e.g., [127, 128]). On the numerical calculations of two-colour ionization of the $\mathrm{H}$ atom see $[129,130]$. Concerning results related to negative ions, we mention that a number of applications of the simplified version (compared with the exact one presented in [55]) of the QQES solution for the $\delta$-model potential in a two-colour field were presented by Becker and co-authors [131] (see also [132]). The two-time Schrödinger equation for QQES, obtained in [55], was used in calculations of multiphoton detachment of $\mathrm{H}^{-}$by two-colour laser fields in [133].

Besides the case of a single atom in a strong monochromatic (or polychromatic) field, a complete set of QES in $R_{3} \oplus T$ is convenient as a basis for the analysis of laser pulse effects and also for the description of an ensemble of atoms in the density matrix approach, when dissipative effects (relaxation) are taken into account. The density matrix for a quantum system in a non-resonant laser field was derived in $[124,134]$ as a solution of the Liouville equation, 
using the QES basis set and taking into account the radiative damping (i.e. the interaction with the electromagnetic vacuum) as a relaxation mechanism. The generalization of the density matrix approach to account for damping effects in an ensemble of finite-level systems subject to an intense laser field was presented in $[135,136]$. Finally, a number of authors have employed the QES formalism to treat the solution of the Schrödinger equation in the case of a laser pulse. Thus non-adiabatic corrections to the QES wavefunctions were obtained in [134], and a number of specific effects caused by the non-monochromaticity of a laser pulse were considered in $[124,134]$. An adiabatic QES basis was introduced in [134] based on the extended, two-time ( $t$ and $\tau$ ) Schrödinger equation, where the (fast) time $t$ acts as a time coordinate in $R_{3} \oplus T$ and $\tau$ is a slow (adiabatic) variable. The authors of [137] call this method the ' $\left(t, t^{\prime}\right)$-formalism' (see also [138]). The extension of the QES formalism to resonant multiphoton excitation and population inversion with pulsed fields was treated in $[136,139]$. Recent applications of the adiabatic QQES basis to the analysis of ionization by a short laser pulse have been given, for example, in $[140,141]$.

Thus, over two decades, up to the mid-1980s, the basic ideas and theorems of the quasienergy approach were formulated. Some of them have been reviewed, for example, in $[6,83,112,124,136,142,143]$. As shown by the above brief (and probably incomplete) review, many researchers have contributed to this achievement. The past decade has seen intensive development of algorithms and methods for non-perturbative numerical calculations in this area, for example, the high-frequency approach $[87,144]$, the use of the Coulomb-Sturmian basis in complex quasienergy calculations $[111,112]$ and the powerful $R$-matrix approach for an accurate account of electron correlation in many-electron atoms [89]. A review of the complex scaling method which includes applications to the complex quasienergy problem is given in [145]. An effective method for the calculation of the complex quasienergy for manyelectron atoms (by means of the 'many-electron, many-photon theory') is reviewed in [146]. At the present time the complex quasienergy (or non-Hermitian Floquet) approach is one of the major methods used in non-perturbative numerical analyses of the interaction of atoms and molecules with a strong (and superstrong) laser field (see, e.g., the recent review [89]). A combination of both the quasistationary and quasienergy (Floquet) approaches, which is called the quasistationary, quasienergy state formalism, provides the most appropriate way to treat a decaying quantum system under the influence of either monochromatic or polychromatic external perturbations. The major advantage of this approach is that its use reduces the initialvalue (Cauchy) problem for the time-dependent Schrödinger equation to a much simpler, eigenvalue problem in an enlarged Hilbert space.

\subsection{Outline of the contents of this review}

In this review we present an exact, general solution for the problem of a weakly bound atomic system (a negative ion, described here by an electron in a short-range, $\delta$-function potential) under the simultaneous action of two strong fields: a static electric field and an arbitrarily polarized monochromatic laser field. The content of the rest of this review is comprised of three major parts, which we discuss in turn.

(a) In section 2 a short description of the basic formal equations of the QQES method is presented, and both perturbative and non-perturbative treatments of the interaction of an atomic system with external fields are given. Section 3 is devoted to the integral eigenvalue equation for the complex quasienergy $\epsilon$ corresponding to the unperturbed binding energy, $E_{0}$, of an electron bound in a $\delta$-model potential and interacting with two strong fields: a static electric field and an elliptically polarized laser field. First, in section 3.1, we present our notation describing the polarization of a laser field and the geometrical arrangement 
of the two external fields. We also present a convenient set of scaled units which permit our results to be applied to an arbitrary negative ion rather than to only one particular one. However, since many previous works have dealt specifically with the $\mathrm{H}^{-}$ion, we specify explicitly the relation of our scaled results to this particular negative ion. Then, in section 3.2 our results for the complex quasienergy, $\epsilon$, and for the QQES wavefunction, $\Phi_{\epsilon}(\boldsymbol{r}, t)$, are obtained. The final results for $\epsilon$ are presented in two forms (see (43) and (44) below), which are useful, respectively, for an exact or a perturbative account of laser-field effects, as well as in both cases an exact account of static electric field effects. These general results simplify in a number of special cases, which are enumerated in section 3.3. Two of the most important are discussed in detail in sections 4 and 5. In section 4 the transcendental equation for the complex energy $E$ of a quasistationary state in a strong static electric field $\mathcal{F}$ is analysed, and simple analytical approximations for $\operatorname{Re} E$ and $\operatorname{Im} E$ in terms of Airy functions are obtained (which have a high accuracy up to $\left.\mathcal{F} \sim F_{0}=\sqrt{2 m\left|E_{0}\right|^{3}} /|e| \hbar\right)$. In section 5 the leading $\left(\propto F^{2}\right)$ term for the laser-fieldinduced correction to the energy $E_{0}, \Delta \epsilon^{\mathrm{opt}}$, in the expansion of the exact result for $\epsilon$ in the amplitude $F$ of the laser field is analysed. Our analysis starts from the Brillouin-Wigner series in $F$ for the complex quasienergy $\epsilon$. The final result for $\Delta \epsilon^{\mathrm{opt}}$ is presented in two equivalent forms, which prove to be convenient for our later analysis of the accuracy of previous approximate photodetachment calculations.

(b) Our derivations in sections 3-5 of the complex energy, $E$, and quasienergy, $\epsilon$, are carried out without explicit use of the QQES wavefunctions. Since use of such wavefunctions for the description of decaying systems in strong external fields is uncommon, because their use requires consideration of a number of theoretical questions, and because such an approach can provide a useful consistency check of our results in sections 3-5, we address the use of the QQES wavefunctions in sections 6 and 7. As for the case of quasistationary (or resonance) states in radiationless atomic problems, the QQES wavefunctions are non-normalizable by standard procedures. In section 6 we address the question of normalization and introduce a set of dual QQES wavefunctions for an arbitrary polarization state of a laser field. For the $\delta$-model potential, the normalization integral for these functions is calculated explicitly for the case of both strong fields, and the result is analysed in more detail for the important case of a QS in a strong static field. In spite of the fact that the normalization factor for QQES is unconventional (e.g. because it is complex), we show in section 6.3 that the direct perturbative (in the laser-field amplitude) calculation of $\Delta \epsilon^{\mathrm{opt}}$ using a basis of quasistationary states in a strong static field gives a result that coincides with that obtained in section 5 using the exact relations for the complex quasienergy $\epsilon$. Using the properly normalized QQES functions, in section 7 the concept of the dipole moment, $\tilde{\boldsymbol{d}}(t)$, of a decaying atomic state (in strong static and/or laser fields) is introduced. After making a connection in section 7.1 to the dynamical Stark effect, in section 7.2 we analyse the case of zero laser field. The corresponding nonlinear (in $\mathcal{F}$ ) static polarizability $\alpha(\mathcal{F})$ is calculated explicitly for the $\delta$-model potential and its connection with the complex energy $E$ in a static field is established based on the Hellmann-Feynman theorem, generalized for use with the quasistationary states. Our definition for $\alpha(\mathcal{F})$ is justified by the fact that using our explicit expression for $\alpha(\mathcal{F})$ the exact equation for $E$ (section 4) is derived once again. In a similar way, in section 7.3 the dynamic polarizability tensor is defined in terms of the Fourier component of $\tilde{\boldsymbol{d}}(t)$ with frequency $\omega$. Two independent components of this tensor, $\alpha_{0}(\mathcal{F} ; \omega)$ and $\alpha_{1}(\mathcal{F} ; \omega)$, are presented in terms of Airy functions for the case of a weak laser field. These quantities describe the linear in $F$ response of an atomic system in a static field to an external monochromatic perturbation in a way similar to that of the ordinary dynamic polarizability 
$\alpha(\omega)$ of a stable system. Specifically, using the generalized Hellmann-Feynman theorem, the linear in laser intensity correction to the quasienergy, $\Delta \epsilon^{\mathrm{opt}}$, obtained in alternative ways in sections 5 and 6 , is presented here in terms of $\alpha_{0}$ and $\alpha_{1}$, whose combination we define as the (generalized) dynamic polarizability $\alpha(\mathcal{F} ; \omega)$. In section 7.4 the cross section for elastic photon scattering by an atomic system in the presence of a static electric field is presented in terms of $\alpha_{0}$ and $\alpha_{1}$ and a new, static-field-induced polarization effect (circular dichroism) is predicted and evaluated numerically for the $\mathrm{H}^{-}$ion. Detailed numerical analyses and simple analytical approximations for $\alpha(\mathcal{F} ; \omega)$ for frequencies near to and far from threshold $\left(\hbar \omega=\left|E_{0}\right|\right)$ are presented in section 8 for the case of the $\delta$-model potential.

(c) In section 9 the photodetachment cross section in the presence of a static electric field, $\sigma(\mathcal{F}, \omega)$, is considered. We define $\sigma(\mathcal{F}, \omega)$ in terms of the imaginary part of the dynamic polarizability $\alpha(\mathcal{F} ; \omega)$, i.e. similarly to the optical theorem for the case $\mathcal{F}=0$. The accuracy of this definition is investigated with respect to the strength of the static field, and, for appropriate values of the static field, the exact analytical result for $\sigma(\mathcal{F}, \omega)$ is presented in section 9.1 in terms of Airy functions and their derivatives. In section 9.2 the separate terms in this result are identified with different levels of approximation used in earlier studies of photodetachment. A number of figures are presented to illustrate the frequency and $\mathcal{F}$ dependence of $\sigma(\mathcal{F}, \omega)$ as well as to compare with prior approximate results for the collinear field geometry. Some special questions, in particular, the polarization and field geometry dependence of $\sigma(\mathcal{F}, \omega)$ and the accuracy of the concept of photodetachment for strong static fields are discussed in section 9.3.

In section 10 we present a summary and some conclusions. Finally, a number of mathematical results employed in this paper are collected in appendices A-C. Appendix A contains results for the various Green functions for an electron in static electric and laser fields. In appendix B are collected some mathematical results concerning integrals involving the Green function for a free electron in a static electric field. In appendix $\mathrm{C}$ are collected a number of expansions for this function and its derivatives.

\section{Basic equations of the QQES approach}

We consider a quantum system initially prepared in a bound state, with wavefunction

$$
\Psi_{n}(\boldsymbol{r}, t)=\psi_{n}(\boldsymbol{r}) \mathrm{e}^{-\frac{\mathrm{i}}{\hbar} E_{n} t}
$$

of the Hamiltonian

$$
H_{0}=T+U \quad T=-\frac{\hbar^{2} \nabla^{2}}{2 m}
$$

under the simultaneous action of a uniform static electric field $\mathcal{F}$ and a monochromatic laser field with an electric field vector $\boldsymbol{F}(t)=F \operatorname{Re}\{e \exp (-\mathrm{i} \omega t)\}$, where $e \cdot e^{*}=1$. We denote the interaction operator in the electric dipole approximation as $V(r, t)$, which in the length gauge has the form

$$
V(\boldsymbol{r}, t)=|e|(\boldsymbol{F}(t)+\mathcal{F}) \cdot \boldsymbol{r} .
$$

For systems subject to a periodic perturbation, the most natural basis set is the complete set of quasienergy states (QES) [5] (or 'steady' [63] or 'Floquet' [59] states),

$$
\Psi_{\epsilon}(\boldsymbol{r}, t)=\Phi_{\epsilon}(\boldsymbol{r}, t) \mathrm{e}^{-\frac{\mathrm{i}}{\hbar} \epsilon t}
$$


where $\Phi_{\epsilon}(\boldsymbol{r}, t)$ are periodic solutions of the 'stationary' Schrödinger equation in the enlarged Hilbert space $R_{3} \oplus T$ [63]

$$
\mathcal{H} \Phi_{\epsilon}=\epsilon \Phi_{\epsilon} \quad \Phi_{\epsilon}(\boldsymbol{r}, t+2 \pi / \omega)=\Phi_{\epsilon}(\boldsymbol{r}, t)
$$

with the QES Hamiltonian

$$
\mathcal{H}=T+U(\boldsymbol{r})+V(\boldsymbol{r}, t)-\mathrm{i} \hbar \frac{\partial}{\partial t} .
$$

For the atomic Hamiltonian $H_{0}$, the quasienergy spectrum is continuous, $\epsilon \geqslant 0$, and the functions $\Phi_{\epsilon}(\boldsymbol{r}, t)$ form a complete set of continuous states normalized to a $\delta$-function in (quasi)energy. This set is particularly useful for analysis of collisional problems or other problems involving quantum transitions in the presence of $V(\boldsymbol{r}, t)$.

If we are interested only in the decay (e.g. by photodetachment, ionization or dissociation) of an initial state (1) caused by $V(\boldsymbol{r}, t)$ and if the fields $\mathcal{F}$ and $\boldsymbol{F}(t)$ are not so strong that depletion is significant, then the transition rate can be introduced, and the problem is simplified by using the concept of quasistationary QES instead of exact QES solutions. In contrast with the QES introduced initially for real values of $\epsilon[5,59,63]$, the QQES are similar to the quasistationary states in a static potential $V(r)$ (see, e.g., $[66,90])$. Such states were initially introduced for the analysis of the shift and broadening of a weakly bound level in a circularly polarized light field [84, 93] (see also [94, 110]). The QQES describe the exponential (in time) decay of bound states of the potential $U(\boldsymbol{r})$ caused by $V(\boldsymbol{r}, t)$; they are also periodic (in time) solutions of the quasienergy equation (5) but with the complex boundary condition (radiation condition) corresponding to outgoing waves at $r \rightarrow \infty$ (cf [90]). Such solutions exist only for definite complex values of the parameter $\epsilon$,

$$
\epsilon_{n}=\operatorname{Re} \epsilon_{n}-\frac{1}{2} \mathrm{i} \Gamma_{n}
$$

If at the adiabatic switching off of $V(\boldsymbol{r}, t) \epsilon_{n}$ tends to the energy $E_{n}$ of the unperturbed state (1), then $\Delta E_{n}=\operatorname{Re} \epsilon_{n}-E_{n}$ and $W_{n}=\Gamma_{n} / \hbar$ are the energy shift and the ionization rate of the state $\Psi_{n}(\boldsymbol{r}, t)$ caused by the potential $V(\boldsymbol{r}, t)$.

A discussion of the exponential decay law for a quantum system in a monochromatic light field analogous to the standard theory of the decay of an initially prepared state in a static potential [91] is presented in [105]. In the quasienergy approach the relation between the scattering resonances, the poles of the continuous spectrum QES wavefunctions in the complex $\epsilon$-plane, and the QQES is similar to that for time-independent Hamiltonians, as was demonstrated in [106] (see also the review [6]). Various forms for the formulation of the eigenvalue problem for the QQES in terms of integral equations are discussed in these cited works based on equation (5) or on the equivalent Lippman-Schwinger equation for the scattering states.

In the perturbation theory (PT) approach, equation (5) for complex $\epsilon$ corresponding to $E_{n}$ may be rewritten in terms of the reduced, 'unperturbed' quasienergetic Green function of an atom,

$$
G_{E}^{a}=\left(1-\left|\psi_{n}\right\rangle\left\langle\psi_{n}\right|\right) \mathcal{G}_{E}^{a}
$$

where

$$
\mathcal{G}_{E}^{a}=\frac{1}{E-\mathcal{H}_{0}}=\sum_{k=-\infty}^{\infty} \sum_{m} \frac{\left|\psi_{m} \mathrm{e}^{\mathrm{i} k \hbar \omega t}\right\rangle\left\langle\psi_{m} \mathrm{e}^{\mathrm{i} k \hbar \omega t^{\prime}}\right|}{E-E_{m}-k \hbar \omega+\mathrm{i} 0}
$$


and where $\mathcal{H}_{0}$ is the unperturbed QES Hamiltonian of an atom, i.e. $\mathcal{H}$ with $V \equiv 0$. Starting from the Lippmann-Schwinger equation for the scattering states of the Hamiltonian $\mathcal{H}$, the equations for $\Phi_{\epsilon_{n}}$ and $\epsilon_{n}$ may be presented as follows [106]:

$$
\begin{aligned}
& \left.\Phi_{\epsilon_{n}}=\psi_{n}+G_{\epsilon_{n}}^{a} V\left|\Phi_{\epsilon_{n}}\right\rangle\right\rangle \\
& \epsilon_{n}=E_{n}+\left\langle\left\langle\psi_{n}|V| \Phi_{\epsilon_{n}}\right\rangle\right\rangle
\end{aligned}
$$

where the double-bracket notation indicates a cycle-averaged scalar product [63]

$$
\left\langle\left\langle\Phi_{1} \mid \Phi_{2}\right\rangle\right\rangle=\frac{1}{T} \int_{0}^{T} \mathrm{~d} t\left\langle\Phi_{1} \mid \Phi_{2}\right\rangle \quad T=\frac{2 \pi}{\omega} .
$$

Equation (12) gives the definition of the scalar product in the space $R_{3} \oplus T$, and the single double bracket in (10) indicates a cycle average over the time $t^{\prime}$ in (9). Based on (10) and (11), the PT for the complex quasienergy may be developed similarly to time-independent PT in terms of the resolvent operator [147] (see also [63] for the case of a stationary QES). The regularization procedure for the elimination of singularities in higher-order matrix elements of PT for complex $\epsilon_{n}$ is discussed in [105].

A convenient way to take account of $V(r, t)$ non-perturbatively in calculations of the complex quasienergy is the following integral eigenvalue equation for $\epsilon$ and $\Phi_{\epsilon}(\boldsymbol{r}, t)$ :

$$
\begin{aligned}
\Phi_{\epsilon}(\boldsymbol{r}, t) & =\int_{-\infty}^{t} \mathrm{~d} t^{\prime} \mathrm{e}^{\mathrm{i} \epsilon\left(t-t^{\prime}\right) / \hbar} \int \mathrm{d} \boldsymbol{r}^{\prime} G\left(\boldsymbol{r}, t ; \boldsymbol{r}^{\prime}, t^{\prime}\right) U\left(\boldsymbol{r}^{\prime}\right) \Phi_{\epsilon}\left(\boldsymbol{r}^{\prime}, t^{\prime}\right) \\
& =\int_{0}^{\infty} \mathrm{d} \tau \mathrm{e}^{\mathrm{i} \epsilon \tau / \hbar} \int \mathrm{d} \boldsymbol{r}^{\prime} G\left(\boldsymbol{r}, t ; \boldsymbol{r}^{\prime}, t-\tau\right) U\left(\boldsymbol{r}^{\prime}\right) \Phi_{\epsilon}\left(\boldsymbol{r}^{\prime}, t-\tau\right) .
\end{aligned}
$$

Here $G\left(\boldsymbol{r}, t ; \boldsymbol{r}^{\prime}, t^{\prime}\right)$ is the (retarded) Green function for a free electron in an external static field $\mathcal{F}$ and an electromagnetic field $\boldsymbol{F}(t)$, which has the well known path integral form of Feynman [148],

$$
\mathrm{i} \hbar G\left(\boldsymbol{r}, t ; \boldsymbol{r}^{\prime}, t^{\prime}\right)=\Theta\left(t-t^{\prime}\right)\left[\frac{m}{2 \pi \mathrm{i} \hbar\left(t-t^{\prime}\right)}\right]^{3 / 2} \exp \left[\frac{\mathrm{i}}{\hbar} S_{\mathrm{cl}}\left(\boldsymbol{r}, t ; \boldsymbol{r}^{\prime}, t^{\prime}\right)\right]
$$

where $S_{\mathrm{cl}}$ is the classical action for the electron in the fields considered here and $\Theta(x)$ is a Heaviside function. Note, that the integral over $\tau\left(t^{\prime}\right)$ in (13) is formally divergent at the upper (lower) limit for $\operatorname{Im} \epsilon<0$ and is to be understood as the analytic continuation from the upper half-plane of complex $\epsilon$, where $\operatorname{Im} \epsilon>0$. The details for choosing the proper sheet of the Riemann surface for this continuation are discussed in [103,106]. The explicit form of $G\left(\boldsymbol{r}, t ; \boldsymbol{r}^{\prime}, t^{\prime}\right)$ for the combination of a static electric field and an elliptically polarized light field is discussed below in section 3.2 and in appendix A. Note that $G$ is normalized according to the equation,

$$
\left(\mathrm{i} \hbar \frac{\partial}{\partial t}+\frac{\hbar^{2} \nabla_{r}^{2}}{2 m}-V(\boldsymbol{r}, t)\right) G\left(\boldsymbol{r}, t ; \boldsymbol{r}^{\prime}, t^{\prime}\right)=\delta\left(\boldsymbol{r}-\boldsymbol{r}^{\prime}\right) \delta\left(t-t^{\prime}\right) .
$$

Equation (13) is exact (in the QQES approach) and, in principle, can be solved for the complex quasienergy in arbitrarily strong fields $\boldsymbol{F}(t)$ and $\mathcal{F}$. A separate problem is the physical meaning of the complex quasienergy in superstrong fields. As was mentioned above, $\Gamma_{n}=-2 \operatorname{Im} \epsilon_{n}$ determines the ionization rate of the bound state (1) in the region of exponential decay. Therefore, in this region the QQES approach is equivalent to the solution of the initialvalue (i.e. Cauchy) problem for the exact time-dependent Schrödinger equation with the initial condition (1). Thus, the reduction of a Cauchy problem for a time-dependent Hamiltonian to a much simpler complex eigenvalue problem is one of the major advantages of the QQES 
approach. On the other hand, the exponential decay law has a relative accuracy $\sim \Gamma_{n} /\left|\operatorname{Re} \epsilon_{n}\right|$ $[91,105]$. Thus, the imaginary part of the quasienergy has physical meaning if it is small compared with the real part, $\left|\operatorname{Re} \epsilon_{n}\right|$. (Note, however, that special considerations are necessary for resonant ionization [149].) If this condition is not satisfied, the QQES approach fails physically, together with the approximation of the exponential decay of a bound level. This gives some limitations on the strength of the external electric fields for which the concept of the transition rate can be used. Evidently, the same limitations exist in all other treatments dealing with the transition rate (e.g. $S$-matrix, etc), even if these limitations are not discussed explicitly. The QQES approach presents an accurate means of calculation of the ionization rate and the energy shift of a bound level, taking exact account of the level shift effects.

\section{Solution of the QQES problem for the zero-range potential in two fields}

The eigenvalue problem for the QQES is simplified in the case of a short-range potential $U(\boldsymbol{r})$ having one bound state, with energy $E_{0}=-\hbar^{2} \kappa^{2} / 2 m$, modelled by the zero-range potential (or ' $\delta$-model' potential)

$$
U(\boldsymbol{r})=\frac{2 \pi \hbar^{2}}{m \kappa} \delta(\boldsymbol{r}) \frac{\partial}{\partial r} r .
$$

The bound-state wavefunction in this model has the well known one-parametric form:

$$
\psi_{0}(\boldsymbol{r})=N \frac{\mathrm{e}^{-\kappa r}}{r}
$$

with the normalization factor, $N=\sqrt{\kappa / 2 \pi}$.

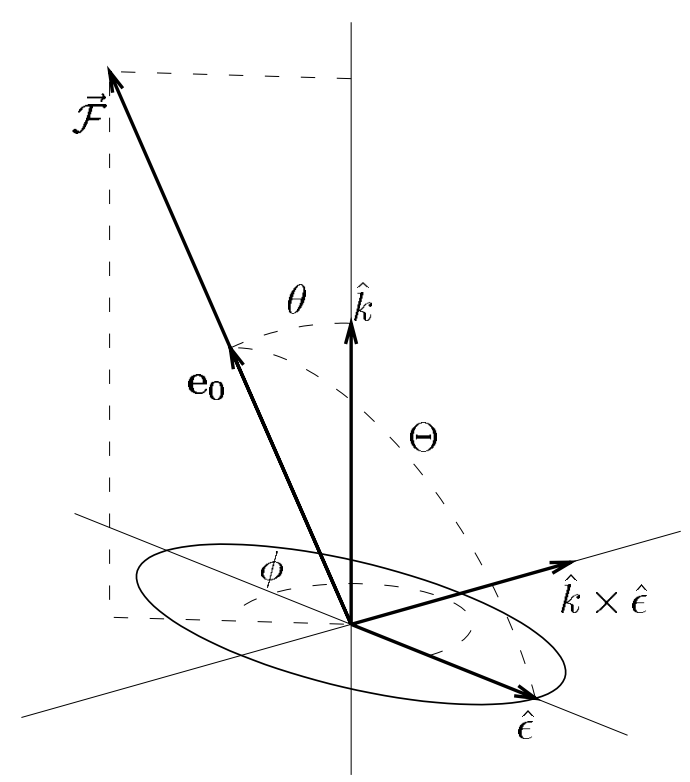

Figure 1. Geometry of a static and a laser field. 


\subsection{Geometry of the fields and definitions of scaled units}

To derive the equations for $\epsilon$ and $\Phi_{\epsilon}(r, t)$, we consider the most general geometry for the applied fields, namely, that the direction of the vector $\mathcal{F}$ is defined by the polar angles $\theta$, $\phi$ in the coordinate frame whose $z$-axis is directed along the wavevector $\boldsymbol{k}=\hat{k} \omega / c$ of the elliptically polarized laser field $\boldsymbol{F}(t)$ and whose $x$-axis is along the major semiaxis of the polarization ellipse, defined by the unit vector $\hat{\epsilon}$. The following parametrization of the unit complex polarization vector $e\left(e \cdot e^{*}=1\right)$ may be used for the general case of an elliptically polarized field $\boldsymbol{F}(t)$ with ellipticity parameter $\eta$ :

$$
e=\frac{\hat{\epsilon}+\mathrm{i} \eta \hat{k} \times \hat{\epsilon}}{\sqrt{1+\eta^{2}}} \quad-1 \leqslant \eta \leqslant 1
$$

where $\eta=0$ corresponds to linear polarization, and $\eta=+1(-1)$ corresponds to right (left) circular polarization. These relationships are illustrated in figure 1 , where the unit vector $\boldsymbol{e}_{0}$ defines the direction of a static field, $\mathcal{F}=\mathcal{F} e_{0}$, and where the angle $\Theta$ is the only one which enters the results for the case of linearly polarized $\boldsymbol{F}(t), \cos \Theta=\sin \theta \cos \phi$. Instead of the ellipticity $\eta$ for the description of photon polarization, it is often convenient to employ the degrees of linear $(l)$ and circular $(\xi)$ polarization:

$$
l=\frac{1-\eta^{2}}{1+\eta^{2}}=e \cdot e=e^{*} \cdot e^{*} \quad \xi=\frac{2 \eta}{1+\eta^{2}}=\mathrm{i} \hat{k} \cdot\left(e \times e^{*}\right) .
$$

Note that $\xi$ and $l$ are connected with the standard Stokes parameters $\xi_{1}, \xi_{2}, \xi_{3}$ (e.g. $\xi=\xi_{2}$, $l=\sqrt{\xi_{1}^{2}+\xi_{3}^{2}}$ ), which are used in the photon density matrix approach [150,151]. $l$ is the degree of maximal linear polarization, i.e. along the major axis of the polarization ellipse. In our notation the (complex) scalar product $\boldsymbol{e} \cdot \mathcal{F}$ can be written as

$$
e \cdot \mathcal{F}=\frac{\mathcal{F} \sin \theta}{\sqrt{2(1+l)}}((1+l) \cos \phi+\mathrm{i} \xi \sin \phi)=|e \cdot \mathcal{F}| \mathrm{e}^{\mathrm{i} \phi_{1}}
$$

where

$$
\begin{aligned}
& |e \cdot \mathcal{F}|=\mathcal{F} \Lambda / \sqrt{2} \quad \Lambda=\sin \theta \sqrt{1+l \cos 2 \phi}=\sin \theta \sqrt{1+\xi_{3}} \\
& \tan \phi_{1} \equiv \tan (\arg (e \cdot \mathcal{F}))=\eta \tan \phi .
\end{aligned}
$$

These formulae demonstrate that all results are invariant with respect to the transformation $\phi \rightarrow \phi-\pi$, i.e. the two opposite orientations of the vector $\hat{\epsilon}$ along the major axis of the polarization ellipse are equivalent.

In order to present the results in this paper in the most general way, we employ a system of units in which the only parameter of the short-range potential ground state, its binding energy, is used to scale all physical variables. In this way our results become independent of this binding energy parameter and thus may be applied to any concrete system that may be described by a short-range potential. More specifically, all energies and the frequency $\omega$ are measured in units of binding energy, $\left|E_{0}\right|$, and its corresponding frequency, $\left|E_{0}\right| / \hbar$, respectively. The unit of length is $\kappa^{-1}=\hbar / \sqrt{2 m\left|E_{0}\right|}$. The strengths of both electric fields are measured in units of the 'internal field'

$$
F_{0}=\sqrt{2 m\left|E_{0}\right|^{3}} /|e| \hbar=\hbar^{2} \kappa^{3} / 2 m|e| .
$$

This field is determined by the condition

$$
d_{0} F_{0}=\left|E_{0}\right| \quad d_{0}=|e| \kappa^{-1}=\frac{|e| \hbar}{\sqrt{2 m\left|E_{0}\right|}}
$$


where $d_{0}$ is the 'internal dipole moment'. The unit of polarizability is $d_{0} / F_{0}=2 m e^{2} / \hbar^{2} \kappa^{4}$. As an example, for $\mathrm{H}^{-}$we have $F_{0}^{\mathrm{H}^{-}}=3.362 \times 10^{7} \mathrm{~V} \mathrm{~cm}^{-1}$; thus $1 \mathrm{MV} \mathrm{cm}^{-1}=0.03 F_{0}^{\mathrm{H}^{-}}$. Note that for the $\mathrm{H}$ atom, $F_{0}^{\mathrm{H}}=\frac{1}{2} F_{\text {at }}$, where $F_{\text {at }}=m^{2} e^{5} / \hbar^{4}=5.142 \times 10^{9} \mathrm{~V} \mathrm{~cm}^{-1}$ is the atomic unit for the strength of the electric field. These scaled units enable the reader to judge how our results apply to any particular atomic system (and also enable us to avoid choosing a particular system to illustrate our results). When required for clarity, however, we shall also present results in absolute units.

We comment now on the application of results in this paper to real negative ions. We employ in this review the standard normalization constant $N=\sqrt{\kappa / 2 \pi}$ for the initial state (17), which is appropriate for the (one-parameter) $\delta$-potential model. However, it is well known that for real atoms or negative ions more exact results may be obtained using, instead of $N$, a corrected normalization constant, $N_{\mathrm{c}}$, which may be obtained, for example, by analysing the asymptotic behaviour of the wavefunction for large $r$ or by an effective range theory analysis (on the recent status of such analyses see [152]). For this case, our results for the (quasi)energy, the polarizability and the photodetachment and photon scattering cross sections should be multiplied by the 'renormalization' factor $A_{\mathrm{c}}=2 \pi N_{\mathrm{c}}^{2} / \kappa$. For $\mathrm{H}^{-}$the factor $A_{\mathrm{c}}$ has the value 2.6551 (see [41] for more details). Further, since we measure the cross section in units of $\kappa^{-2}$, in order to convert it to au, $\sigma(\omega)$ should be multiplied by the ratio $\left|E_{\mathrm{H}} / E_{0}\right|$, where $\left|E_{\mathrm{H}}\right|=0.5$ au is the binding energy of hydrogen. For $\mathrm{H}^{-}$this ratio is 18.017 . Accounting also for $A_{\mathrm{c}}$, the combined scaling factor between $\sigma^{\mathrm{H}^{-}}(\omega)$ (in au) and our $\sigma(\omega)$ (in units of $\kappa^{-2}$ ) is 47.838 , i.e.

$$
\sigma^{\mathrm{H}^{-}} \quad(\text { in } \mathrm{au})=47.837 \sigma^{\mathrm{H}^{-}}(\omega) \quad \text { (in scaled units). }
$$

Similarly, the factor relating the polarizabilities $\alpha^{\mathrm{H}^{-}}(\mathcal{F} ; \omega)$ (in au) and $\alpha(\mathcal{F} ; \omega)$ (in units $\left.2 m e^{2} / \hbar^{2} \kappa^{4}\right)$ is $2 A_{\mathrm{c}}\left(E_{\mathrm{H}} / E_{0}\right)^{2}=1723.8$, i.e.

$$
\alpha^{\mathrm{H}^{-}}(\mathcal{F} ; \omega) \quad(\text { in au })=1723.8 \alpha(\mathcal{F} ; \omega) \quad \text { (in scaled units). }
$$

In particular, for the static polarizability of $\mathrm{H}^{-}$, the scaled result in (118) gives 215.5 au upon application of (23), which agrees with the recommended value 206 au [153] (see also [154]).

\subsection{Exact results for $\epsilon$ and $\Phi_{\epsilon}(\boldsymbol{r}, t)$ in scaled units}

As for the analysis of static-field ionization [3,155], the key simplification in the QQES problem for the $\delta$-potential model is due to the known boundary condition for a solution of the Schrödinger equation involving the short-range potential (16),

$$
\left.\Phi_{\epsilon}(\boldsymbol{r}, t)\right|_{r \rightarrow 0}=\left(\frac{1}{r}-\kappa\right) f_{\epsilon}(t) .
$$

For $V \equiv 0$, the function $f_{\epsilon}$ is simply the normalization factor $N=\sqrt{\kappa / 2 \pi}$ of the bound-state wavefunction (17). For a time-dependent interaction $V(\boldsymbol{r}, t)$, the function $f_{\epsilon}(t)$ represents the time-periodic part of $\left.\Phi_{\epsilon}(\boldsymbol{r}, t)\right|_{r \rightarrow 0}$. Moreover, the Fourier spectrum of $f_{\epsilon}(t)$ depends on the symmetry of the QES Hamiltonian. For the special case of circularly polarized light, $f_{\epsilon}$ is also a constant $[84,93]$ and (13) therefore reduces to a transcendental equation for $\epsilon$ that is similar to that for the case of a static field [155]. For linearly or elliptically polarized light, $f_{\epsilon}(t)$ contains only even Fourier components [25]. Finally, for a combination of static and light fields with an arbitrary geometry, the function $f_{\epsilon}(t)$ involves both odd and even harmonics, as discussed below.

The relation

$$
U\left(\boldsymbol{r}^{\prime}\right) \Phi_{\epsilon}\left(\boldsymbol{r}^{\prime}, t\right)=-\frac{2 \pi \hbar^{2}}{m} \delta\left(\boldsymbol{r}^{\prime}\right) f_{\epsilon}(t)
$$


makes it possible to perform the integrations over $r^{\prime}$ in (13). Further, taking $r \rightarrow 0$ in that equation and using relation (24), allows one to derive a one-dimensional integral equation for $f_{\epsilon}(t)$ and $\epsilon$ which contains only the classical action for zero radial variables, $S_{\mathrm{cl}}\left(\boldsymbol{r}=0, t ; \boldsymbol{r}^{\prime}=0, t-t^{\prime}\right) \equiv S_{\mathrm{cl}}\left(t, t-t^{\prime}\right)$. The Green function $G\left(\boldsymbol{r}, t ; \boldsymbol{r}^{\prime}, t^{\prime}\right)$ for a free electron in both a static field and a monochromatic light field is presented in appendix A. In terms of the units introduced above, the action $S_{\mathrm{cl}}\left(t, t-t^{\prime}\right)$ for our problem is as follows (cf [25]):

$$
\begin{aligned}
S_{\mathrm{cl}}\left(t, t-t^{\prime}\right)= & S_{\mathrm{cl}}^{F}\left(t, t-t^{\prime}\right)-\frac{1}{12} \mathcal{F}^{2} t^{\prime 3} \\
& +\Lambda \frac{4 \gamma}{\omega^{2}} \mathcal{F} \cos \left(\omega t-\frac{1}{2} \omega t^{\prime}-\phi_{1}\right)\left(\frac{1}{2} \omega t^{\prime} \cos \frac{1}{2} \omega t^{\prime}-\sin \frac{1}{2} \omega t^{\prime}\right) .
\end{aligned}
$$

Here $S^{F}$ is the action for an elliptically polarized light field,

$$
S_{\mathrm{cl}}^{F}\left(t, t-t^{\prime}\right)=-\frac{\gamma^{2}}{\omega}\left[\omega t^{\prime}\left(1-\frac{4 \sin ^{2} \frac{1}{2} \omega t^{\prime}}{\left(\omega t^{\prime}\right)^{2}}\right)-l \cos \omega\left(2 t-t^{\prime}\right)\left(\sin \omega t^{\prime}-\frac{4 \sin ^{2} \frac{1}{2} \omega t^{\prime}}{\omega t^{\prime}}\right)\right] .
$$

The geometry and polarization dependences are described by the three parameters, $l, \Lambda$ and $\phi_{1}$. The important parameter $\gamma$ is defined by the relation

$$
\gamma^{2}=\frac{F^{2}}{2 \omega^{2}}=E_{F} \quad\left(=\frac{e^{2} F_{\mathrm{abs}}^{2}}{4 m \omega_{\mathrm{abs}}{ }^{2}\left|E_{0}\right|} \quad \text { in absolute units }\right)
$$

which shows that $\gamma^{2}$ is the ratio of the quiver energy of an electron in a light field to the binding energy. This parameter is related to the well known Keldysh parameter [126], $\gamma_{K}$, of strong field theories according to the relation, $\gamma^{2}=\left(2 \gamma_{K}^{2}\right)^{-1}$. For the case of linearly polarized light, equations (26) and (27) agree with equation (20) of [52].

With the above definitions, the final equation for $f_{\epsilon}(t)$ and $\epsilon$ has the form

$\left(\sqrt{E^{\prime}}-1\right) f_{\epsilon}(t)=(4 \pi \mathrm{i})^{-1 / 2} \int_{0}^{\infty} \frac{\mathrm{d} t^{\prime}}{t^{\prime 3 / 2}} \mathrm{e}^{-\mathrm{i} E^{\prime} t^{\prime}}\left\{f_{\epsilon}\left(t-t^{\prime}\right) \mathrm{e}^{\mathrm{i}\left[S_{\mathrm{cl}}\left(t, t-t^{\prime}\right)+\gamma^{2} t\right]}-f_{\epsilon}(t)\right\}$

where

$$
E^{\prime}=E_{F}-\epsilon
$$

(Note that $\epsilon=E_{0}=-1$ for $F, \mathcal{F}=0$.) Equation (29) for $\mathcal{F}=0$ was analysed in detail in [25]. For a non-zero static field this equation can be transformed into a more convenient form. For this purpose we introduce, firstly, the new function, $\phi_{\epsilon}(t)$, instead of $f_{\epsilon}(t)$ :

$$
f_{\epsilon}(t)=\phi_{\epsilon}(t) \exp \left[\mathrm{i} \frac{l \gamma^{2}}{2 \omega} \sin 2 \omega t-\mathrm{i} \Lambda \frac{2 \gamma \mathcal{F}}{\omega^{2}} \sin \left(\omega t-\phi_{1}\right)\right] .
$$

This substitution removes those terms which are periodic in time from $S_{\mathrm{cl}}\left(0, t ; 0, t-t^{\prime}\right)$ and is equivalent to the unitary transformation of the wavefunction of a free electron in a static and a laser field [51], which removes the term $\propto A^{2}(t)$ in the photon-atom interaction together with the cross term $\propto \mathcal{F} F$. We then add and subtract the term $\phi_{\epsilon}(t) \exp \left(-\mathrm{i} \mathcal{F}^{2} t^{\prime 3} / 12\right)$ within the braces of the integrand in (29). The final equation for $\epsilon$ and $\phi_{\epsilon}(t)$ becomes

$$
\left[1-g_{\mathcal{F}}\left(-E^{\prime}\right)\right] \phi_{\epsilon}(t)=-(4 \pi \mathrm{i})^{-1 / 2} \int_{0}^{\infty} \frac{\mathrm{d} t^{\prime}}{t^{\prime 3 / 2}} \mathrm{e}^{-\mathrm{i} E^{\prime} t^{\prime}-\mathrm{i} \mathcal{F}^{2} t^{33} / 12}\left\{\phi_{\epsilon}\left(t-t^{\prime}\right) \mathrm{e}^{\mathrm{i} \Delta\left(t, t^{\prime}\right)}-\phi_{\epsilon}(t)\right\}
$$


where

$\Delta\left(t, t^{\prime}\right)=\frac{4 \gamma^{2} \sin ^{2} \frac{1}{2} \omega t^{\prime}}{\omega^{2} t^{\prime}}\left[1-l \cos \omega\left(2 t-t^{\prime}\right)\right]+\Lambda \frac{2 \gamma \mathcal{F}}{\omega^{2}} \cos \left(\omega t-\frac{1}{2} \omega t^{\prime}-\phi_{1}\right) \omega t^{\prime} \cos \frac{1}{2} \omega t^{\prime}$.

In the derivation of (32), we used the following integral identity (see appendix B):

$$
-(4 \pi \mathrm{i})^{-1 / 2} \int_{0}^{\infty} \frac{\mathrm{d} t^{\prime}}{t^{\prime 3 / 2}} \mathrm{e}^{-\mathrm{i} E^{\prime} t^{\prime}}\left(\mathrm{e}^{-\mathrm{i} \mathcal{F}^{2} t^{\prime 3} / 12}-1\right)=-\sqrt{E^{\prime}}+g_{\mathcal{F}}\left(-E^{\prime}\right)
$$

for the 'regularized' part, $g_{\mathcal{F}}(E)$, of the stationary Green function, $G_{E}\left(\boldsymbol{r}, \boldsymbol{r}^{\prime}\right)$, of an electron in a static electric field $\mathcal{F}[24,52]$ :

$$
g_{\mathcal{F}}(E)=4 \pi \lim _{r \rightarrow 0}\left[G_{E}(\boldsymbol{r} ; 0)+\frac{1}{4 \pi r}\right]=-\pi \mathcal{F}^{1 / 3} J\left(-E / \mathcal{F}^{2 / 3}\right)
$$

where

$$
J(\xi) \equiv \operatorname{Ai}^{\prime}(\xi) \mathrm{Ci}^{\prime}(\xi)-\xi \operatorname{Ai}(\xi) \operatorname{Ci}(\xi) .
$$

Here $\operatorname{Ci}(\xi) \equiv \operatorname{Bi}(\xi)+\mathrm{i} \operatorname{Ai}(\xi)$, where $\operatorname{Ai}(\xi)$ and $\operatorname{Bi}(\xi)$ are the regular and irregular Airy functions [156].

Expanding $\phi(t)$ in a Fourier series,

$$
\phi_{\epsilon}(t)=\sum_{n=-\infty}^{\infty} \phi_{n} \mathrm{e}^{\mathrm{i} n \omega t}
$$

we obtain an infinite system of linear homogeneous equations for $\phi_{n}$ and the quasienergy $\epsilon$,

$$
\left[1+\pi \mathcal{F}^{1 / 3} J\left(\xi_{n}^{\prime}\right)\right] \phi_{n}=\sum_{n^{\prime}=-\infty}^{\infty} M_{n n^{\prime}}\left(E^{\prime}\right) \phi_{n^{\prime}}
$$

where $\xi_{n}^{\prime}=\left(E^{\prime}+n \omega\right) \mathcal{F}^{-2 / 3}, \xi_{0}^{\prime} \equiv \xi^{\prime}$.

The matrix $M_{n n^{\prime}}$ is defined by

$$
\begin{aligned}
M_{n n^{\prime}}\left(E^{\prime}\right)= & \sqrt{\frac{\omega}{4 \pi \mathrm{i}}} \int_{0}^{\infty} \frac{\mathrm{d} \tau}{\tau^{3 / 2}} \exp \left\{-\mathrm{i}\left[\left(\frac{E^{\prime}}{\omega}+\frac{n+n^{\prime}}{2}\right) \tau+\frac{\mathcal{F}^{2} \tau^{3}}{12 \omega^{3}}\right]\right\} \\
& \times\left\{\delta_{n^{\prime} n}-\exp \left[\mathrm{i} \frac{4 \gamma^{2}}{\omega} \frac{\sin ^{2} \tau / 2}{\tau}\right] K_{n^{\prime}-n}(\tau)\right\}
\end{aligned}
$$

where $K$ is defined in terms of the Bessel functions $J_{v}(x)$ as

$$
K_{m}(\tau)=\sum_{k=-\infty}^{\infty} \mathrm{i}^{m+k} J_{k}(z(\tau)) J_{m-2 k}(\tilde{\gamma}(\tau)) \mathrm{e}^{\mathrm{i}(m-2 k) \phi_{1}} .
$$

Here

$$
z(\tau)=l \frac{4 \gamma^{2}}{\omega} \frac{\sin ^{2} \tau / 2}{\tau}
$$

and

$$
\tilde{\gamma}(\tau)=\frac{2 \Lambda \gamma \mathcal{F}}{\omega^{2}} \tau \cos \frac{1}{2} \tau .
$$


We note the important general symmetry relations for $M$ 's,

$$
\begin{aligned}
& M_{n^{\prime}+l, n+l}\left(E^{\prime}\right)=M_{n^{\prime}, n}\left(E^{\prime}+l \omega\right) \\
& M_{n, n^{\prime}}(\eta)=M_{n^{\prime}, n}(-\eta) \quad M_{-n,-n^{\prime}}(\eta, \omega)=M_{n, n^{\prime}}(-\eta,-\omega)
\end{aligned}
$$

and an additional relation valid only for the case of a linearly polarized field $\boldsymbol{F}(t)$,

$$
M_{n, n^{\prime}}\left(E^{\prime}\right)=M_{n^{\prime}, n}\left(E^{\prime}\right) .
$$

The above equations permit a reduction in the number of required calculations for $M_{n, n^{\prime}}$ to only those for the case $M_{k, 0}$ with $k \geqslant 0$.

It follows from (38) that the quasienergy $\epsilon$ can be calculated numerically as a solution of the transcendental equation given by the Fredholm determinant of the linear system in (38),

$$
\text { Det }\left\|\left[1+\pi \mathcal{F}^{1 / 3} J\left(\xi_{n}^{\prime}\right)\right] \delta_{n n^{\prime}}-M_{n n^{\prime}}\left(E^{\prime}\right)\right\|=0
$$

with the boundary condition $\epsilon=E_{0}=-1$ at $F, \mathcal{F}=0$. For $\gamma<1$ we have $M_{n n^{\prime}}\left(E^{\prime}\right) \sim \gamma^{\left|n-n^{\prime}\right|+2 \delta_{n n^{\prime}}}$, and thus in this case the solution of (38) can be obtained iteratively by means of the Brillouin-Wigner series

$$
\begin{aligned}
1+\pi \mathcal{F}^{1 / 3} J\left(\xi^{\prime}\right) & =M_{00}\left(-E^{\prime}\right)+\sum_{n \neq 0} \frac{M_{0, n}\left(-E^{\prime}\right) M_{n, 0}\left(-E^{\prime}\right)}{1+\pi \mathcal{F}^{1 / 3} J\left(\xi_{n}^{\prime}\right)} \\
& +\sum_{n \neq 0} \sum_{m \neq 0} \frac{M_{0, n}\left(-E^{\prime}\right) M_{n, m}\left(-E^{\prime}\right) M_{m, 0}\left(-E^{\prime}\right)}{\left(1+\pi \mathcal{F}^{1 / 3} J\left(\xi_{n}^{\prime}\right)\right)\left(1+\pi \mathcal{F}^{1 / 3} J\left(\xi_{m}^{\prime}\right)\right)}+\cdots
\end{aligned}
$$

Equations (43) and (44) allow one to calculate $\epsilon$ for arbitrary $\gamma$. For large $\gamma$, the result (43) must be used, while for $\gamma<1$, an iterative (perturbative) approach using (44) is the most appropriate.

Finally, we note that for known $\epsilon$ and known Fourier coefficients of the function $f_{\epsilon}(t)$ in (24) and (25), the QQES wavefunction $\Phi_{\epsilon}(r, t)$ may be written (up to a normalization factor which is an overall factor for Fourier coefficients of $f_{\epsilon}\left(t^{\prime}\right)$, see section 6.2 for details) in accordance with (13) as

$$
\Phi_{\epsilon}(\boldsymbol{r}, t)=-4 \pi \int_{-\infty}^{t} \mathrm{~d} t^{\prime} \mathrm{e}^{\mathrm{i} \epsilon\left(t-t^{\prime}\right)} G\left(\boldsymbol{r}, t ; \boldsymbol{r}^{\prime}=0, t^{\prime}\right) f_{\epsilon}\left(t^{\prime}\right) .
$$

Taking into account (31), this equation may also be rewritten in an equivalent form in terms of the function $\phi_{\epsilon}\left(t^{\prime}\right)$.

\subsection{Some special cases}

The main difficulty in the solution of the general equations (43) and (44) for the quasienergy $\epsilon$ is the calculation of the matrix elements $M_{n, n^{\prime}}\left(E^{\prime}\right)$ according to (39) and (40), especially since equation (40) for $K_{m}(\tau)$ involves an infinite summation. The problem simplifies, however, in a few special cases.

(a) For $\mathcal{F} \rightarrow 0$ we have $g_{\mathcal{F}}\left(-E^{\prime}\right) \rightarrow \sqrt{E^{\prime}}$ (see appendix C, equation (C3)), $K_{m}(\tau)$ equals a single Bessel function with argument $z(\tau)$, and equations (32), (43) and (44) coincide with the corresponding equations for an elliptic field given in [25].

(b) The interference between static and light fields vanishes for orthogonal geometry, $\boldsymbol{F}(t) \cdot \mathcal{F}=0$ (or $\Lambda=0$ ). As is evident from (32) and (33), in this case all of the dependence on the static field is given by the exponential factor $\exp \left(-\mathrm{i} \mathcal{F}^{2} t^{\prime 3} / 12\right)$. 
(c) Interference effects are most important for the coplanar geometry in which case $\mathcal{F}$ lies in the plane of light polarization (i.e. $\theta=\pi / 2$ ) and are maximal for a linearly polarized $\boldsymbol{F}(t)$ collinear with the static field. The latter case has been analysed in [52].

(d) For circular polarization $K_{m}(\tau)$ reduces to

$$
K_{m}(\tau)=\mathrm{i}^{m} \mathrm{e}^{\mathrm{i} \xi m \phi} J_{m}\left(\sin \theta \frac{2 \gamma \mathcal{F}}{\omega^{2}} \tau \cos \frac{1}{2} \tau\right)
$$

although one should note that $\phi_{\epsilon}(t)$ contains both even and odd Fourier components. Only for orthogonal fields $(\sin \theta=0)$ does $K_{m}(\tau)=\delta_{m 0}$, so that the results become similar to the case of a circularly polarized field [84,93], differing only by an additional 'static-field' factor $\exp \left(-\mathrm{i} \mathcal{F}^{2} t^{\prime 3} / 12\right)$. This case was analysed previously in [24].

An additional special case is that in which the laser field is zero, $\boldsymbol{F}(t)=0$. We treat this case briefly in the next section; that is, we analyse the complex energy of a weakly bound level in a static field.

\section{Complex energy of QS in a static electric field}

For $F=0$ we have $M_{n m}=0$ and the result obtained above in (44) gives the complex energy $E \equiv \epsilon$ of a quasistationary state in a static field $\mathcal{F}$ :

$$
1+\pi \mathcal{F}^{1 / 3} J\left(-\frac{E}{\mathcal{F}^{2 / 3}}\right)=0 .
$$

This is equivalent to the result of [155], where another form for the electron Green function in a static field was used. Formally, equation (47) is valid for an arbitrary $\mathcal{F}$ and can be solved numerically as a transcendental equation with Airy functions (see (36)). However, the results can be presented in a simple analytic form for a wide range of static fields, up to $\mathcal{F} \leqslant 1$.

For weak fields, $\mathcal{F} \ll 1$, we use the asymptotic expansion in $(\mathrm{C} 3)$ to obtain the well known result [155]

$$
\Delta E^{\text {stat }}(\mathcal{F} \ll 1)=E-E_{0}=-\frac{1}{16} \mathcal{F}^{2}-\mathrm{i} \frac{1}{4} \mathcal{F} \mathrm{e}^{-4 / 3 \mathcal{F}} .
$$

For a stronger field, $\mathcal{F} \leqslant 1$, the simplest way to obtain a non-perturbative solution of (47) is to expand $J$ in a Taylor series in $\Delta E=E-E_{0}$. This series is convergent for $|\Delta E| \equiv\left|E-E_{0}\right|<1$ as a consequence of the analyticity of the Airy functions. Taking into account only terms linear in $\Delta E$ in the Taylor expansion for $J$ at $E=E_{0} \equiv-1$, we find

$$
\Delta E^{\text {stat }}=-\frac{1+\pi \mathcal{F}^{1 / 3} J\left(\mathcal{F}^{-2 / 3}\right)}{\pi \mathcal{F}^{-1 / 3} I\left(\mathcal{F}^{-2 / 3}\right)}
$$

where

$$
I(\xi)=-J^{\prime}(\xi)=\operatorname{Ci}(\xi) \operatorname{Ai}(\xi)
$$

and the prime denotes the derivative with respect to the argument. Some properties of these functions are discussed in appendices B and C.

Equation (49), where both $J$ and $I$ are complex, demonstrates the strong coupling between the real and the imaginary parts of the complex energy $E$. Therefore, for a strong field $\mathcal{F}$ the position and width of the quasistationary level cannot be determined independently since they are the real and imaginary parts of the same analytical (in $\mathcal{F}$ ) function having an essential 
singularity at $\mathcal{F}=0$. Using the explicit forms for $J(\xi)$ and $I(\xi)$, we may express the real and imaginary parts of $E$ in terms of the Airy functions $\operatorname{Ai}(\xi)$ and $\operatorname{Bi}(\xi)$ :

$$
\begin{aligned}
& \operatorname{Re} E=-\frac{\mathcal{F}^{1 / 3}}{\pi \operatorname{Ai}(\xi) M(\xi)}\left[\operatorname{Bi}(\xi)+\frac{1}{2} \pi \mathcal{F}^{1 / 3} \operatorname{Ai}^{\prime}(\xi) M^{\prime}(\xi)\right] \\
& \Gamma \equiv-2 \operatorname{Im} E=-\frac{2 \mathcal{F}^{1 / 3}}{\pi \operatorname{Ai}(\xi) M(\xi)}\left[\operatorname{Ai}(\xi)+\mathcal{F}^{1 / 3} \operatorname{Ai}^{\prime}(\xi)\right]
\end{aligned}
$$

where $\xi=\mathcal{F}^{-2 / 3}, M(\xi)=\mathrm{Ai}^{2}(\xi)+\mathrm{Bi}^{2}(\xi)$. For $\mathcal{F} \rightarrow 0$, the result in (51) and (52) coincides with that corresponding to (48). We also mention here the result for $\Gamma$ obtained in [51], which is more accurate than that given by (48) but not as accurate as that given by (52). Namely, calculating the integral in equation (70) of [51] (see (B17)), we find

$$
\Gamma=4 \pi \mathcal{F}^{1 / 3}\left[\mathrm{Ai}^{\prime 2}(\xi)-\xi \mathrm{Ai}^{2}(\xi)\right]=4 \pi \mathcal{F}^{1 / 3} \operatorname{Im} J(\xi) .
$$

In our approach, this result follows from (49) by neglecting the $\mathcal{F}$ dependence of the denominator on the right-hand side of this equation, which is proportional to the normalization factor of the initial-state wavefunction in a static field (see (95) below). Obviously, the level shift obtained by using this approximation in (49) is

$$
\begin{aligned}
\operatorname{Re} \Delta E^{\text {stat }} & =-2\left(1+\pi \mathcal{F}^{1 / 3} \operatorname{Re} J(\xi)\right) \\
& =-2\left(1+\pi \mathcal{F}^{1 / 3}\left[\operatorname{Ai}^{\prime}(\xi) \operatorname{Bi}^{\prime}(\xi)-\xi \operatorname{Ai}(\xi) \operatorname{Bi}(\xi)\right]\right)
\end{aligned}
$$

Table 1 presents a numerical comparison of these various results for $\operatorname{Re} \Delta E^{\text {stat }}$ and $\Gamma$, i.e. the solution of the exact transcendental equation (47) versus the use of the approximate equations (48)-(54). The accuracy of the approximate result in (51) and (52) is seen to be very high for all reasonable values of $\mathcal{F}$ at which $\Gamma /|\operatorname{Re} E| \ll 1$. The results in table 1 also

\begin{tabular}{|c|c|c|c|c|}
\hline \multirow{3}{*}{$\begin{array}{l}\text { Static field } \\
\mathcal{F}\end{array}$} & \multicolumn{4}{|c|}{ Level shift, $\Delta E$} \\
\hline & \multirow{2}{*}{$\begin{array}{l}\text { Exact result } \\
\text { equation (47) }\end{array}$} & \multicolumn{3}{|c|}{ Approximate results } \\
\hline & & Equation (51) & Equation (54) & Equation (48) \\
\hline 0.03 & $-5.6326(-5)$ & $-5.6326(-5)$ & $-5.6337(-5)$ & $-5.6260(-5)$ \\
\hline 0.06 & $-2.2625(-4)$ & $-2.2624(-4)$ & $-2.2637(-4)$ & $-2.2500(-4)$ \\
\hline 0.15 & $-1.4664(-3)$ & $-1.4658(-3)$ & $-1.4716(-3)$ & $-1.4063(-3)$ \\
\hline 0.30 & $-6.4831(-3)$ & $-6.4719(-3)$ & $-6.5827(-3)$ & $-5.6250(-3)$ \\
\hline \multirow[t]{2}{*}{1.00} & $-4.4162(-2)$ & $-4.4417(-2)$ & $-4.1217(-2)$ & $-6.2500(-2)$ \\
\hline & \multicolumn{4}{|c|}{ Level width, $\Gamma$} \\
\hline \multirow{2}{*}{$\begin{array}{l}\text { Static field } \\
\mathcal{F}\end{array}$} & \multirow{2}{*}{$\begin{array}{l}\text { Exact result } \\
\text { equation (47) }\end{array}$} & \multicolumn{3}{|c|}{ Approximate results } \\
\hline & & Equation (52) & Equation (53) & Equation (48) \\
\hline 0.03 & $7.3027(-22)$ & $7.3024(-22)$ & $7.3314(-22)$ & $7.4837(-22)$ \\
\hline 0.06 & $6.3861(-12)$ & $6.3852(-12)$ & $6.4388(-12)$ & $6.7009(-12)$ \\
\hline 0.15 & $9.2092(-6)$ & $9.2004(-6)$ & $9.4329(-6)$ & $1.0343(-5)$ \\
\hline 0.30 & $1.4022(-3)$ & $1.3958(-2)$ & $1.4926(-3)$ & $1.7615(-3)$ \\
\hline 1.00 & $7.7609(-2)$ & $7.6041(-2)$ & $8.8265(-2)$ & $1.3180(-1)$ \\
\hline
\end{tabular}
demonstrate the importance of properly taking into account the normalization factor of the (quasistationary) wavefunction in a strong enough static field.

Table 1. Level shifts and widths for a bound state in a short-range potential in the presence of a static electric field.

${ }^{\text {a }}$ Scaled units, see section 3.1 . 
Equation (47) is also convenient for the investigation of the case of superstrong fields, $\mathcal{F} \gg 1$. Such results are interesting for exploring the general analytical structure of the bound state energy and width as a function of $\mathcal{F}$ in strong fields (see, e.g., [157, 158]). For this case we rewrite (47) as

$$
J\left(-\frac{E}{\mathcal{F}^{2 / 3}}\right)=-\frac{1}{\pi \mathcal{F}^{1 / 3}}
$$

and assume $E=\tilde{E}+\Delta \tilde{E}$, where $|\Delta \tilde{E} / \tilde{E}| \ll 1$, and $\tilde{E}$ obeys the equation

$$
J\left(-\xi_{0}\right)=0 \quad \xi_{0}=\frac{\tilde{E}}{\mathcal{F}^{2 / 3}} .
$$

The numerical solution of this equation gives (for the solution $\xi_{0}$ having $\operatorname{Im} \tilde{E}<0$ ):

$$
\xi_{0}=0.44133122 \mathrm{e}^{-\mathrm{i} \pi / 3} \text {. }
$$

The expression for $\Delta \tilde{E}$ follows from (55), taking into account the first two terms in the Taylor expansion of the left-hand side, for $E=\tilde{E}$. The resulting asymptotic expression for $E$ is

$$
E=\xi_{0} \mathcal{F}^{2 / 3}-\frac{\mathcal{F}^{1 / 3}}{\pi I\left(\xi_{0}\right)}+\cdots
$$

where $I\left(\xi_{0}\right)=0.36871854 \mathrm{e}^{\mathrm{i} \frac{1}{6} \pi}$. Thus, for superstrong fields the complex energy can be presented as a series in $\mathcal{F}^{-1 / 3}$. The main term $\left(\propto \mathcal{F}^{2 / 3}\right)$ in (57) for the three-dimensional $\delta$ well was calculated numerically in [158]. The two-terms given in (57) are sufficient provided $\left|\pi \xi_{0} I\left(\xi_{0}\right) \mathcal{F}^{1 / 3}\right| \gg 1$. Numerically this gives $\mathcal{F} \gg 10$.

As is seen from (57), for large $\mathcal{F}$ both $|\operatorname{Im} E|$ and $|\operatorname{Re} E|$ are of the same order of magnitude and $\Gamma$ cannot be treated as an ionization probability per unit time. Moreover, $\operatorname{Re} E>0$, and thus as $\mathcal{F}$ increases the quasistationary state transforms into a virtual state leading to an $S$ matrix pole in the upper half-plane of complex $E$. Note that the asymptotic form of $E$ for the one-dimensional $\delta$-potential [157], $E \sim \exp (-\mathrm{i} \pi / 3)(\mathcal{F} \ln \mathcal{F})^{2 / 3}$, contains the logarithmic term typical for one-dimensional problems. We note that for the ground state of the hydrogen atom in a strong static electric field [159], $E \sim \exp (-\mathrm{i} \pi / 3)(\mathcal{F} \ln \mathcal{F})^{2 / 3}$, which has the same phase and dependence on $\mathcal{F}$ as the result for the one-dimensional $\delta$-potential.

\section{Weak-laser-field expansion of the exact result for $\epsilon$}

The exact equation (44) can be solved analytically for the important case of a low-intensity laser field. Indeed, in contrast with a perturbation theory (PT) expansion for the energy in a static field, which is a real (asymptotic) expansion and, therefore, cannot be used for the determination of the complex part of the energy (the level width, which is exponentially small for a weak static field), a PT expansion in the laser field is convergent, even when a (possibly strong) static field is present. In particular, the PT expansion for the quasienergy has a finite radius of convergence $[106,116]$. Thus, for a weak $\boldsymbol{F}(t)$, accurate PT expansions can be derived, both for the real and the imaginary parts of $\epsilon$.

For finite frequencies, the matrix elements (39) are analytic functions of $F$ at $F=0$. Therefore, they can be expanded in a power series in $F$, and, for weak fields such that $\gamma<1$, we may retain only the first non-vanishing terms in $F$ in the Taylor expansions for $M_{n n^{\prime}}$. Only $M_{0,0}$ and $M_{0, \pm 1}$ contribute in this case, and they are proportional to $F^{2}$ and $F$, respectively. Their explicit form is (see appendix B for further details):

$$
\begin{aligned}
& M_{ \pm 1,0}\left(E^{\prime}\right)=-\mathrm{e}^{\mp \mathrm{i} \phi_{1}} \Lambda \frac{\pi \gamma \mathcal{F}^{2 / 3}}{2 \omega}\left(I\left(\xi^{\prime}\right)+I\left(\xi_{ \pm 1}^{\prime}\right)\right) \\
& M_{ \pm 1,0}\left(E^{\prime}\right) \mathrm{e}^{ \pm \mathrm{i} \phi_{1}}=M_{0, \pm 1}\left(E^{\prime}\right) \mathrm{e}^{\mp \mathrm{i} \phi_{1}}
\end{aligned}
$$


$M_{0,0}\left(E^{\prime}\right)=-\frac{2 \pi \gamma^{2} \mathcal{F}}{3 \omega^{2}} \sum_{n=-1}^{1}(-1)^{n} C_{2}^{n+1}\left[\xi_{n}^{\prime} J\left(\xi_{n}^{\prime}\right)+\frac{1}{4}\left(1-(-1)^{n} \frac{3}{2} \Lambda^{2}\right) I^{\prime}\left(\xi_{n}^{\prime}\right)\right]$

where $C_{2}^{n+1}$ is the binomial coefficient. Equation (44) thus reduces to

$1+\pi \mathcal{F}^{1 / 3} J\left(\xi^{\prime}\right)=M_{00}\left(E^{\prime}\right)+\frac{M_{0,1}\left(E^{\prime}\right) M_{1,0}\left(E^{\prime}\right)}{1+\pi \mathcal{F}^{1 / 3} J\left(\xi_{+1}^{\prime}\right)}+\frac{M_{0,-1}\left(E^{\prime}\right) M_{-1,0}\left(E^{\prime}\right)}{1+\pi \mathcal{F}^{1 / 3} J\left(\xi_{-1}^{\prime}\right)}$.

Using the analyticity of $J(\operatorname{cf}(36))$ and its derivatives, $I(\operatorname{cf}(50))$ and $I^{\prime}$, we can write $E^{\prime}=-\epsilon$ (cf (30)) on the right-hand side of (60), since taking account of the ponderomotive shift $E_{F}$ (cf (28)) here is superfluous to lowest order in $F$. On the left-hand side, we use an expansion valid for small $\gamma^{2}$ :

$J\left(\xi^{\prime}\right) \approx J(\xi)-\gamma^{2} \mathcal{F}^{-2 / 3} I(\xi) \quad \xi_{n}=\frac{-\epsilon+n \omega}{\mathcal{F}^{2 / 3}} \quad \xi \equiv \xi_{0}=\frac{-\epsilon}{\mathcal{F}^{2 / 3}}$.

As a result, we find the following transcendental equation for the complex quasienergy $\epsilon=E_{0}+\Delta \epsilon$ :

$$
1+\pi \mathcal{F}^{1 / 3} J(\xi)=\pi \gamma^{2} \mathcal{F}^{-1 / 3} I(\xi)+\Sigma(-\epsilon)
$$

where

$$
\Sigma(-\epsilon) \equiv M_{00}(-\epsilon)+\frac{M_{0,1}(-\epsilon) M_{1,0}(-\epsilon)}{1+\pi \mathcal{F}^{1 / 3} J\left(\xi_{+1}\right)}+\frac{M_{0,-1}(-\epsilon) M_{-1,0}(-\epsilon)}{1+\pi \mathcal{F}^{1 / 3} J\left(\xi_{-1}\right)} .
$$

Equation (62) is our result for the quasienergy that is accurate to the first non-vanishing terms in $F$ and that takes exact account of static field effects. Two limiting cases follow immediately: for $\mathcal{F}=0$, we obtain the result for the quasienergy up to quadratic terms in $F$ in an elliptically polarized laser field [25], whose imaginary part is connected with the photodetachment cross section for $\omega>\left|E_{0}\right|$; for $F=0$, we obtain the result (47) for the complex energy in a static field.

The most accurate way to solve equation (62) for $\Delta \epsilon \equiv \epsilon-E_{0}$ analytically is to substitute $\epsilon \simeq E \equiv E_{0}+\Delta E^{\text {stat }}$ in the right-hand side of (62) (where $\Delta E^{\text {stat }}$ is given, for example, by (49)) and to find the laser-field-induced correction, $\Delta \epsilon^{\mathrm{opt}} \equiv \epsilon-E=\Delta \epsilon-\Delta E^{\text {stat }}$, in a way similar to that used in the static field case to obtain (49). As result, we find the linear in laser intensity correction to the energy $E_{0}$ of the bound state (17) in a static and in a (weak) laser field:

$$
\epsilon-E_{0} \equiv \Delta \epsilon=\Delta E^{\mathrm{stat}}+\Delta \epsilon^{\mathrm{opt}}
$$

where

$$
\Delta \epsilon^{\mathrm{opt}}=\gamma^{2}+\Sigma(-E) / \pi \mathcal{F}^{-1 / 3} I\left(-E / \mathcal{F}^{2 / 3}\right)
$$

and where the function $\Sigma(-E)$ is defined by the right-hand side of (63) with $\epsilon=E$. However, such an approach is necessary only for a strong enough field, $\mathcal{F} \leqslant 1$, when field ionization is important. Thus, for a weak field $\mathcal{F}$, we can write with reasonable accuracy $E \simeq E_{0}=-1$ in the right-hand side of (65). Taking into account the explicit form of the matrix elements $M$ in equation (63) for $\Sigma$, it is seen that, as for the case of a static field in section 4 , the analytic result for $\Delta \epsilon^{\mathrm{opt}}$ involves again only combinations of Airy functions and their derivatives. The frequency dependence of $\Delta \epsilon^{\mathrm{opt}}$ is concentrated in the arguments of these functions for a weak field $\mathcal{F}$, i.e. $(1+n \omega) / \mathcal{F}^{2 / 3}$ with $n=0, \pm 1(\operatorname{cf}(61))$. As mentioned, for higher accuracy we should replace $(1+n \omega)$ by $\left(1+\Delta E^{\text {stat }}+n \omega\right)$ in these arguments. 
In order to discuss more precisely in section 9 the various approximate calculations of the photodetachment cross section, we discuss briefly below an alternative derivation of the result (65) for $\Delta \epsilon^{\mathrm{opt}}$ to that given in section 3.2. Namely, we solve (29) without using the substitution (31). One obtains an equation for $\epsilon$ and $f_{n}$ similar to equation (38) for $\epsilon$ and $\phi_{n}$, but instead of the matrix elements $M_{n n^{\prime}}(\mathrm{cf}(39))$ on the right-hand side one has matrix elements $\bar{M}_{n n^{\prime}}$, which are much more complicated. Despite this complexity, this approach may be preferable for an approximate, non-perturbative (in the laser field) analysis of the complex quasienergy $\epsilon$. For example, in the adiabatic limit of small frequencies, keeping only the matrix element $\bar{M}_{00}$, accurate results for the ionization width (in the absence of the static field), $\Gamma$, were obtained in [25]. Obviously, exact results are independent of the substitution (31); more specifically, the Brillouin-Wigner series for $\epsilon$ again has the form (44), but with $M_{n m} \rightarrow \bar{M}_{n m}$. We do not present here the explicit form of $\bar{M}_{n m}$ for an arbitrary $F$, and present only the results for $\bar{M}_{n m}$ with $n=0,1$ and $m=0$ up to the first non-vanishing order in $F$ (these matrix elements are the only ones which contribute to $\Delta \epsilon^{\mathrm{opt}}$ ):

$\bar{M}_{ \pm 1,0}(-E)=M_{ \pm 1,0}(-E) \pm \mathrm{e}^{\mp \mathrm{i} \phi_{1}} \frac{\Lambda \pi \gamma \mathcal{F}^{4 / 3}}{\omega^{2}}\left(J(\xi)-J\left(\xi_{ \pm 1}\right)\right)$

$\bar{M}_{0,0}(-E)=M_{00}(-E)$

$$
-\Lambda^{2} \frac{\pi \gamma^{2} \mathcal{F}^{5 / 3}}{\omega^{3}}\left[\frac{\mathcal{F}^{2 / 3}}{\omega}\left(J\left(\xi_{-1}\right)-2 J(\xi)+J\left(\xi_{+1}\right)\right)-I\left(\xi_{-1}\right)+I\left(\xi_{+1}\right)\right] .
$$

Compared with $M$ (cf (58) and (59)), we see that the matrix elements $\bar{M}$ involve additional terms with the characteristic factor $\mathcal{F}^{2 / 3} / \omega$. Thus, we find the following equation for $\Delta \epsilon^{\mathrm{opt}}$ (cf (63) and (65)):

$$
\Delta \epsilon^{\mathrm{opt}}=\gamma^{2}+\bar{\Sigma}(-E) / \pi \mathcal{F}^{-1 / 3} I\left(-E / \mathcal{F}^{2 / 3}\right)
$$

where

$\bar{\Sigma}(-E)=\bar{M}_{0,0}(-E)+\frac{\bar{M}_{0,1}(-E) \bar{M}_{1,0}(-E)}{1+\pi \mathcal{F}^{1 / 3} J\left(\xi_{+1}\right)}+\frac{\bar{M}_{0,-1}(-E) \bar{M}_{-1,0}(-E)}{1+\pi \mathcal{F}^{1 / 3} J\left(\xi_{-1}\right)}$.

After some algebra together with the use of (47), it may be verified that $\bar{\Sigma}(-E)=\Sigma(-E)$, and thus the result (68) is equivalent to that in equation (65).

A simple analysis of the frequency dependence of $\Delta \epsilon^{\mathrm{opt}}$ in (65) based on the explicit forms (58) and (59) for the matrix elements $M_{k 0}$, or on the equivalent expression (68), shows that for $\omega \gg 1$ we have $\Delta \epsilon^{\mathrm{opt}} \simeq \gamma^{2}$. Thus, for this case static-field-induced effects are negligible and $\Delta \epsilon^{\mathrm{opt}}$ coincides with the well known ponderomotive shift of bound levels in a high-frequency laser field [60]. For $\omega \ll 1$ the term $\gamma^{2}$ on the right-hand side of (65) is compensated by the last term, involving $\Sigma(-E)$, and $\Delta \epsilon^{\text {opt }}$ provides only a small correction, $\propto F^{2} \mathcal{F}^{2}$, to the results for a static field presented in section 4 . In contrast, for near-threshold frequencies (either above or below), $\omega \approx 1$, the static field changes drastically both the real and the imaginary parts of $\Delta \epsilon$.

\section{The normalization procedure for QQES and PT calculation of $\Delta \epsilon^{\mathrm{opt}}$ in the basis of quasistationary states}

\subsection{Linear in $F$ perturbative expansion of $\Phi_{\epsilon}(r, t)$}

In the derivation of $\Delta \epsilon^{\text {opt }}$ above we have not dealt with QQES wavefunctions as such, exploiting rather the expansion (44) for $\epsilon$. As an example of how the function $\Phi_{\epsilon}(r, t)$ in (13) should be 
employed in concrete calculations, we present here an alternative derivation of $\Delta \epsilon^{\mathrm{opt}}$ based on a perturbation theory treatment of the laser-atom interaction $V_{L}(\operatorname{cf}(10)$ and (11)). We use the length-gauge form for $V_{L}$,

$$
V_{L}(\boldsymbol{r}, t)=\frac{1}{2} F\left(e \cdot r \mathrm{e}^{-\mathrm{i} \omega t}+e^{*} \cdot r \mathrm{e}^{\mathrm{i} \omega t}\right) .
$$

The interaction of the atom with the static field is assumed to be taken into account exactly, both in $\left|\psi_{n}\right\rangle \equiv\left|\Phi_{E}\right\rangle$ and in $E_{n} \equiv E$, both of which enter equations (10) and (11) for $\epsilon_{n} \equiv \epsilon$. Thus, we illustrate below how perturbation theory using a basis of quasistationary states (in a static field) may be used for the calculation of laser-field-induced corrections to the complex energy $E$ of the quasistationary level.

Obviously only three terms of the Fourier expansion for $\Phi_{\epsilon}(\boldsymbol{r}, t)$ in (10) contribute to the second-order correction for $\epsilon$ in (11):

$$
\Phi_{\epsilon}(\boldsymbol{r}, t) \simeq \Phi^{0}(\boldsymbol{r})+\Phi^{-1}(\boldsymbol{r}) \mathrm{e}^{-\mathrm{i} \omega t}+\Phi^{+1}(\boldsymbol{r}) \mathrm{e}^{\mathrm{i} \omega t} .
$$

Moreover, in accordance with (11), in order to calculate $\epsilon$ up to order $F^{2}$, only the terms $\propto F$ on the right-hand side of equation (71) should be included. In this approximation $\Phi^{0}=\Phi_{E}$, and explicit expressions for $\Phi^{0}(\boldsymbol{r})$ and $\Phi^{ \pm 1}(\boldsymbol{r})$ follow from (45) upon expanding the integrand in powers of $F$. Note that

$$
\Phi_{E}(\boldsymbol{r}) \equiv \Phi^{0}(\boldsymbol{r})=\frac{f_{0}}{\sqrt{4 \pi \mathrm{i}}} \int_{0}^{\infty} \frac{\mathrm{d} \tau^{\prime}}{\tau^{\prime 3 / 2}} \mathrm{e}^{\mathrm{i}\left(S^{(0)}\left(\boldsymbol{r}, \tau^{\prime}\right)+E \tau^{\prime}\right)}
$$

where

$$
S^{(0)}(\boldsymbol{r}, \tau) \equiv S^{\text {stat }}\left(\boldsymbol{r}, t ; \boldsymbol{r}^{\prime}=0, t-\tau\right)=\frac{\boldsymbol{r}^{2}}{4 \tau}-\frac{\mathcal{F} \cdot \boldsymbol{r} \tau}{2}-\frac{\mathcal{F}^{2} \tau^{3}}{12}
$$

is the classical action for an electron in a static field, and we express $f_{\epsilon}(t)$ as

$$
f_{\epsilon}(t)=\sum_{-\infty}^{\infty} f_{k} \mathrm{e}^{\mathrm{i} k \omega t} \simeq f_{0}+f_{-1} \mathrm{e}^{-\mathrm{i} \omega t}+f_{+1} \mathrm{e}^{\mathrm{i} \omega t} .
$$

The expression for $\Phi^{-1}$ is

$$
\begin{aligned}
\Phi^{-1}(\boldsymbol{r})=\frac{1}{\sqrt{4 \pi \mathrm{i}}} & \left\{f_{-1} \int_{0}^{\infty} \frac{\mathrm{d} \tau}{\tau^{3 / 2}} \mathrm{e}^{\mathrm{i}\left(S^{(0)}(\boldsymbol{r}, \tau)+E \tau+\omega \tau\right)}\right. \\
+ & f_{0} \frac{1}{2} \mathrm{i} \int_{0}^{\infty} \frac{\mathrm{d} \tau}{\tau^{3 / 2}} \mathrm{e}^{\mathrm{i}\left(S^{(0)}(\boldsymbol{r}, \tau)+E \tau\right)}\left[\frac{4 \gamma \Lambda \mathcal{F}}{\omega^{2}}\left(\frac{1}{2} \omega \tau \cos \frac{1}{2} \omega \tau-\sin \frac{1}{2} \omega \tau\right) \mathrm{e}^{\mathrm{i} \omega \tau / 2} \mathrm{e}^{\mathrm{i} \phi_{1}}\right. \\
& \left.\left.+\mathrm{i} \sqrt{2} \gamma(\boldsymbol{r} \cdot \boldsymbol{e})\left(\frac{2}{\omega \tau} \sin \frac{1}{2} \frac{\omega \tau}{\mathrm{e}}{ }^{\mathrm{i} \omega \tau / 2}-1\right)\right]\right\}
\end{aligned}
$$

The expression for $\Phi^{+1}(\boldsymbol{r})$ follows from that for $\Phi^{-1}(\boldsymbol{r})$ upon making the substitutions $\omega \rightarrow-\omega$ and $\eta \rightarrow-\eta$ (cf (18)) or $\phi_{1} \rightarrow-\phi_{1}$ (cf (20)). The coefficients $f_{-1}$ and $f_{+1}$ may be expressed in terms of $f_{0}$ :

$$
f_{-1}=f_{0} \frac{\Lambda \gamma \mathcal{F}^{2 / 3} \pi}{2 \omega} \mathrm{e}^{\mathrm{i} \phi_{1}}\left[\frac{2 \mathcal{F}^{1 / 3}}{\pi \omega}-\frac{I(\xi)+I\left(\xi_{-1}\right)}{1+\pi \mathcal{F}^{1 / 3} J\left(\xi_{-1}\right)}\right]
$$

and $f_{+1}=f_{-1}(\eta \rightarrow-\eta ; \omega \rightarrow-\omega)$. Note, that equation (75) for $\Phi^{-1}(\boldsymbol{r})$ involves both a contribution originating from the expansion of $G$ (i.e. the term with $f_{0}$ ) and one originating from $f_{\epsilon}(t)$ in (45) (i.e. the term with $\left.f_{-1}\right)$. Moreover, the function $\Phi_{E}(\boldsymbol{r})$ in (72) can be written in terms of the stationary Green function, $G_{E}\left(\boldsymbol{r}, \boldsymbol{r}^{\prime}\right)$, of the free electron in a static field $\mathcal{F}$ (see appendix A) as follows:

$$
\Phi_{E}(\boldsymbol{r})=-4 \pi f_{0} G_{E}(\boldsymbol{r}, 0) .
$$




\subsection{Normalization of the QQES wavefunctions}

The coefficient $f_{0}$ in the above formulae is, in fact, the normalization factor for the function $\psi_{n} \equiv \Phi_{E}$ in (10) and (11). Moreover, it is also the same factor for the function $\Phi_{\epsilon}(r, t)$ in (71) when one takes into account only terms linear in $F$. In this connection it is important to note that equations (10) and (11) were derived initially by assuming that $\psi_{n}$ is the normalized wavefunction of a bound state for the atomic Hamiltonian $H_{0}$ in (2). However, for the present case, $\Phi_{E}(\boldsymbol{r})$ is the quasistationary state of the Hamiltonian $H=H_{0}+\mathcal{F} \cdot \boldsymbol{r}$, and $E$ includes a negative imaginary part, $E=\operatorname{Re} E-\frac{1}{2} \mathrm{i} \Gamma$. The Hamiltonian $H$ for a QS state is notself-adjoint, and, therefore, the standard normalization procedure is inapplicable for $\Phi_{E}(\boldsymbol{r})$ (and, more generally, for $\left.\Phi_{\epsilon}(\boldsymbol{r}, t)\right)$ since the normalization integral is divergent in view of the unboundedness of $\Phi_{E}(r)$ (or $\Phi_{\epsilon}(r, t)$ ) at $r \rightarrow \infty$. This fact is well known in the theory of QS (or 'resonance') states for radiationless problems in atomic and nuclear physics. Different approaches are used for the proper normalization of QS states (see, e.g., [66, 160-162], and a rather extensive literature cited therein). Although differing in their details, almost all of them use the idea of an analytical continuation of the standard normalization procedure used for the bound states to the QS (or resonance) states. In the most rigorous treatments, the space of 'dual' functions $(\tilde{\psi})$ is introduced for the QS states $\psi$ in order that the proper normalization condition is determined by the scalar product $\langle\tilde{\psi} \mid \psi\rangle$ (see, e.g., [163]). As was pointed out in [164] (see also [161, 162]), the proper normalization of the QS state may be fixed by its definition in terms of the residue of the total Green function of a decaying system, $\mathcal{G}_{\mathcal{E}}$, at a resonance pole $\mathcal{E}=\mathcal{E}_{r}$,

$$
\left|\psi_{r}\right\rangle\left\langle\tilde{\psi}_{r}\right|=\lim _{\mathcal{E} \rightarrow \mathcal{E}_{r}}\left(\mathcal{E}-\mathcal{E}_{r}\right) \mathcal{G}_{\mathcal{E}}
$$

where the dual bra vector $\left\langle\tilde{\psi}_{r}\right|$ is, in general, not simply the complex conjugate of $\left|\psi_{r}\right\rangle$. Nevertheless, for the simplest case of radial (or other one-dimensional) problems, the function $\psi$ may be used for $\tilde{\psi}^{*}$. Thus, for this case the proper normalization condition consists in the calculation of the normalization integral without complex conjugation of the (complex) radial wavefunction [165].

For the QQES wavefunctions the normalization problem is complicated by the time dependence of both $\Phi_{\epsilon}(r, t)$ and $V(r, t)$. Only a few authors have discussed this question and suggested some prescriptions for normalization (see, e.g., [112, 166, 167]). Generally, the problem is to define, based on the known QQES solution, $\Phi_{\epsilon}(r, t)$, the 'dual' function, $\tilde{\Phi}_{\epsilon}(r, t)$, which satisfies the same Schrödinger equation as $\Phi_{\epsilon}$ (except, perhaps, for another boundary condition) and provides the proper normalization of $\Phi_{\epsilon}$ in accordance with the relation (cf (12))

$$
\left\langle\left\langle\tilde{\Phi}_{\epsilon} \mid \Phi_{\epsilon}\right\rangle\right\rangle=\frac{1}{T} \int_{0}^{T} \mathrm{~d} t\left\langle\tilde{\Phi}_{\epsilon} \mid \Phi_{\epsilon}\right\rangle=1 .
$$

Thus, the function $\left\langle\tilde{\Phi}_{\epsilon}\right|=\tilde{\Phi}_{\epsilon}^{*}$ may be considered as a 'bra analogue' of $\Phi_{\epsilon} \equiv\left|\Phi_{\epsilon}\right\rangle$ and should be used (instead of $\left\langle\Phi_{\epsilon}\right|$ ) in calculations of the normalization factor and, therefore, of matrix elements (including expectation values) of operators acting on the QQES wavefunctions. For strong laser-atom interactions, these (normalized) functions are necessary, for example, for the calculation of partial rates of ionization with the absorption of a fixed number of photons, or for the calculation of the angular distributions of the escaping electrons [112]. Such functions are also used in an accurate calculation of high-harmonic generation by atoms from an intense laser field [168]. Evidently, the concrete form of $\tilde{\Phi}_{\epsilon}$ depends on the symmetry properties of the problem, i.e. on the structure of the Hamiltonian $H=H_{0}+V(\boldsymbol{r}, t)$. We analyse this problem based on the integral equation (13) for $\Phi_{\epsilon}$ and on the explicit form for the Green function 
(14) (see also appendix A). For the combination of a static electric field and a laser field, the classical action $S\left(\boldsymbol{r}, t ; \boldsymbol{r}^{\prime}, t^{\prime}\right)$ depends on the ellipticity $\eta$ (or on the circular polarization degree $\xi)$ of the laser field through the 'angle' $\phi_{1}$, whose tangent is proportional to $\eta$ (see, e.g., (26)). Thus, in general the QQES wavefunction is also $\eta$-dependent, $\Phi_{\epsilon}=\Phi_{\epsilon, \eta}$, and the following relation for $S=S_{\eta}$ is valid (see (A11)):

$$
S_{\eta}\left(\boldsymbol{r}, t ; \boldsymbol{r}^{\prime}, t^{\prime}\right)=-S_{-\eta}\left(\boldsymbol{r},-t ; \boldsymbol{r}^{\prime},-t^{\prime}\right) .
$$

Let us define the 'dual' function $\tilde{\Phi}_{\epsilon}$ as follows:

$$
\tilde{\Phi}_{\epsilon}(\boldsymbol{r}, t)=\left[\Phi_{\epsilon,-\eta}(\boldsymbol{r},-t)\right]^{*} .
$$

To obtain the equation satisfied by $\tilde{\Phi}_{\epsilon}$ we apply the operations prescribed on the right-hand side of (81) to the integral equation (13). Taking into account the definition (14) for the retarded Green function, $G$, we find (in (82)-(84) absolute units are used)

$$
\begin{aligned}
\Phi_{\epsilon,-\eta}^{*}(\boldsymbol{r},-t)= & \frac{\mathrm{i}}{\hbar} \int_{-\infty}^{\infty} \mathrm{d} t^{\prime} \mathrm{e}^{\mathrm{i} \epsilon^{*}\left(t+t^{\prime}\right) / \hbar} \Theta\left(-t-t^{\prime}\right)\left[\frac{m}{2 \pi \mathrm{i} \hbar\left(t+t^{\prime}\right)}\right]^{3 / 2} \\
& \times \int \mathrm{d} \boldsymbol{r}^{\prime} \exp \left[-\frac{\mathrm{i}}{\hbar} S_{\mathrm{cl},-\eta}\left(\boldsymbol{r},-t ; \boldsymbol{r}^{\prime}, t^{\prime}\right)\right] U\left(\boldsymbol{r}^{\prime}\right) \Phi_{\epsilon,-\eta}^{*}\left(\boldsymbol{r}^{\prime}, t^{\prime}\right) .
\end{aligned}
$$

Changing $t^{\prime}$ to $-t^{\prime}$ in the integrand, and using (80) and the following definition of the advanced Green function:

$$
-\mathrm{i} \hbar G^{(\text {adv })}\left(\boldsymbol{r}, t ; \boldsymbol{r}^{\prime}, t^{\prime}\right)=\Theta\left(t^{\prime}-t\right)\left[\frac{m}{2 \pi \mathrm{i} \hbar\left(t-t^{\prime}\right)}\right]^{3 / 2} \exp \left[\frac{\mathrm{i}}{\hbar} S_{\mathrm{cl}}\left(\boldsymbol{r}, t ; \boldsymbol{r}^{\prime}, t^{\prime}\right)\right]
$$

we obtain

$$
\tilde{\Phi}_{\epsilon}(\boldsymbol{r}, t)=\int_{t}^{\infty} \mathrm{d} t^{\prime} \mathrm{e}^{\mathrm{i} \epsilon^{*}\left(t-t^{\prime}\right) / \hbar} \int \mathrm{d} \boldsymbol{r}^{\prime} G^{(\mathrm{adv})}\left(\boldsymbol{r}, t ; \boldsymbol{r}^{\prime}, t^{\prime}\right) U\left(\boldsymbol{r}^{\prime}\right) \tilde{\Phi}_{\epsilon}\left(\boldsymbol{r}^{\prime}, t^{\prime}\right) .
$$

It is seen that $\tilde{\Phi}_{\epsilon}(\boldsymbol{r}, t)$ satisfies the same Schrödinger equation (5) as $\Phi_{\epsilon}(\boldsymbol{r}, t)$ but with another boundary condition: the ingoing wave boundary condition. As a consequence of using the advanced Green function instead of the retarded one, the function $\tilde{\Phi}_{\epsilon}$ corresponds, in fact, to the eigenvalue $\tilde{\epsilon}=\epsilon^{*}$ (see the exponent in (84)). The definition (81) of $\tilde{\Phi}_{\epsilon}$ can also be used for the 'dual' total QQES wavefunction (4). Then we have

$$
\tilde{\Psi}_{\epsilon}(\boldsymbol{r}, t)=\mathrm{e}^{-\mathrm{i} \epsilon^{*} t} \tilde{\Phi}_{\epsilon}(\boldsymbol{r}, t)
$$

and the following identity for the expectation value of an operator $A$ in the QQES:

$$
\left\langle\tilde{\Psi}_{\epsilon}|A| \Psi_{\epsilon}\right\rangle=\left\langle\tilde{\Phi}_{\epsilon}|A| \Phi_{\epsilon}\right\rangle
$$

This result is consistent with the fact that in the quasienergy approach the time dependence for all physical quantities is only the periodic one. Note that, omitting the complexity of $\epsilon$, the formal transformations from $\Phi_{\epsilon}$ to $\tilde{\Phi}_{\epsilon}$ considered above are similar to those presented in [52] for the construction of the ingoing (or (-)) scattering state in the quasienergy approach starting from the outgoing (or (+)) scattering state with real (continuous) quasienergies.

Our definition of $\tilde{\Phi}_{\epsilon}$ agrees with that suggested by Potvliege and Shakeshaft $[112,166]$, who also argue that such a procedure provides the proper normalization of QQES wavefunctions $\Phi_{\epsilon}$. In $[112,166]$, the authors deal with the numerical solution of the QQES equation (5) for the hydrogen atom. They tested the correctness of the normalization condition in (79) and (81) only numerically and encouraged others to provide additional justification of the normalization convention for the QQES. We present here such an analytical justification for the case of a 
short-range potential, based on (a) the direct calculation of the normalization factor for the case when both fields are strong, and on (b) the comparison of standard PT calculations for $\Delta \epsilon^{\mathrm{opt}}$ with the results (65) or (68), which were obtained using the exact solution for $\epsilon$. The dual function $\tilde{\Phi}_{\epsilon}(r, t)$ for this case is

$$
\tilde{\Phi}_{\epsilon}(\boldsymbol{r}, t)=-4 \pi \int_{t}^{\infty} \mathrm{d} t^{\prime} \mathrm{e}^{\mathrm{i} \epsilon^{*}\left(t-t^{\prime}\right)} G^{(\mathrm{adv})}\left(\boldsymbol{r}, t ; \boldsymbol{r}^{\prime}=0, t^{\prime}\right) \tilde{f}_{\epsilon}\left(t^{\prime}\right) .
$$

This equation can be obtained either by solving (84) similarly to the procedure used to obtain $\Phi_{\epsilon}$ in section 3.2 or by the direct use of the prescription in (81) applied to $\Phi_{\epsilon}(\boldsymbol{r}, t)$ in (45). Since for the time-periodic function $f_{\epsilon}(t)$ the substitution $t \rightarrow-t$ is equivalent to $\omega \rightarrow-\omega$, we have the following relation between Fourier coefficients of $f_{\epsilon}$ and $\tilde{f}_{\epsilon}$ :

$$
\left[\tilde{f}_{k}\right]^{*}=f_{-k}(\omega \rightarrow-\omega) \text {. }
$$

Using explicit forms (45) and (87) for $\Phi_{\epsilon}$ and $\tilde{\Phi}_{\epsilon}$, and taking into account (A9) for the Green functions, it may be verified that the normalization integral $\left\langle\left\langle\tilde{\Phi}_{\epsilon} \mid \Phi_{\epsilon}\right\rangle\right.$ in (79) is actually convergent and can be calculated explicitly. Expressing $\Phi_{\epsilon}$ and $\tilde{\Phi}_{\epsilon}$ in terms of $\phi_{\epsilon}(t)$ (obtained by substituting (31) into (45) and (87)), the normalization condition (79) may be presented as

$$
2 \sqrt{\frac{\pi \mathrm{i}}{\omega}} \sum_{n, k} \tilde{\phi}_{n-k}^{*} \phi_{n} \int_{0}^{\infty} \frac{\mathrm{d} \tau}{\tau^{1 / 2}} \mathrm{e}^{-\mathrm{i}[n-k / 2+\alpha(\tau)] \tau} K_{k}(\tau)=1
$$

where

$$
\alpha(\tau)=\frac{E^{\prime}}{\omega}-\frac{4 \gamma^{2} \sin ^{2}(\tau / 2)}{\omega \tau^{2}}+\frac{\mathcal{F}^{2} \tau^{2}}{12 \omega^{3}}
$$

and where $K_{m}(\tau)$ is given by (40). Similarly, expressing $\Phi_{\epsilon}$ and $\tilde{\Phi}_{\epsilon}$ in terms of $f_{\epsilon}(t)$, as in (45) and (87), we obtain

$$
2 \sqrt{\frac{\pi \mathrm{i}}{\omega}} \sum_{n, k} \tilde{f}_{n-k}^{*} f_{n} \int_{0}^{\infty} \frac{\mathrm{d} \tau}{\tau^{1 / 2}} \mathrm{e}^{-\mathrm{i}[n-k / 2+\alpha(\tau)] \tau} \bar{K}_{k}(\tau)=1
$$

where $\bar{K}_{k}(\tau)$ has the same form as $K_{m}(\tau)$ in (40), but with the following substitutions:

$$
\begin{aligned}
& z(\tau) \rightarrow \bar{z}(\tau)=-\frac{\gamma^{2} l}{\omega}\left(\sin \tau-\frac{4 \sin ^{2} \tau / 2}{\tau}\right) \\
& \tilde{\gamma}(\tau) \rightarrow \overline{\tilde{\gamma}}(\tau)=\frac{4 \Lambda \gamma \mathcal{F}}{\omega^{2}}\left(\frac{1}{2} \tau \cos \frac{1}{2} \tau-\sin \frac{1}{2} \tau\right) .
\end{aligned}
$$

The Fourier coefficients of $f_{\epsilon}(t)$ and $\phi_{\epsilon}(t)$ obey homogeneous equations (see (38)), so that we may write $f_{n}$ as $f_{0} \overline{f_{n}}$ with $\overline{f_{0}}=1$ and similarly for $\phi_{n}$. Then equations (90) or (89) permit one to calculate the bilinear 'normalization parameters' $\left[\tilde{f}_{0}\right]^{*} f_{0}$ or $\left[\tilde{\phi}_{0}\right]^{*} \phi_{0}$. Note that the relations $\left[\tilde{\phi}_{0}\right]^{*}=\phi_{0}$ and $\left[\tilde{f}_{0}\right]^{*}=f_{0}$ are valid only for a linearly polarized laser field. For an elliptically polarized laser field,

$$
\left[\tilde{f}_{k}\right]^{*}=\left.f_{k}\right|_{\eta \rightarrow-\eta} .
$$

Thus for this case it is impossible to determine $f_{0}$ and $\tilde{f}_{0}$ separately. Nevertheless, this does not matter for applications since only the above-mentioned bilinear parameters enter when calculating any physical observables.

To first order in $F$, the normalization integral $\left\langle\left\langle\tilde{\Phi}_{\epsilon} \mid \Phi_{\epsilon}\right\rangle\right\rangle$ for the wavefunction (71) gives

$$
\int \mathrm{d} \boldsymbol{r} \tilde{\Phi}_{E}^{*}(\boldsymbol{r}) \Phi_{E}(\boldsymbol{r})=1
$$


where in accordance with (81), (72) and (77) the dual function for the quasistationary state $\Phi_{E}(\boldsymbol{r})$ is

$$
\tilde{\Phi}_{E}(\boldsymbol{r})=-\frac{\tilde{f}_{0}}{\sqrt{4 \pi \mathrm{i}}} \int_{-\infty}^{0} \frac{\mathrm{d} \tau^{\prime \prime}}{\tau^{\prime \prime 3 / 2}} \mathrm{e}^{\mathrm{i}\left(S^{(0)}\left(\boldsymbol{r}, \tau^{\prime \prime}\right)+E^{*} \tau^{\prime \prime}\right)}=-4 \pi \tilde{f}_{0} G_{E}^{*}(\boldsymbol{r}, 0) .
$$

Thus, equation (92) may be used for the determination of the normalization factors $f_{0}$ and $\tilde{f}_{0}=f_{0}^{*}$ in (72) and (93). The integration over $\boldsymbol{r}$ in (92) is carried out using the following identity:

$$
\begin{gathered}
\int \mathrm{d} \boldsymbol{r} \exp \left[-\mathrm{i}\left(\frac{r^{2}}{4 \tau^{\prime}}-\frac{\mathcal{F} \cdot \boldsymbol{r} \tau^{\prime}}{2}-\frac{\mathcal{F}^{2} \tau^{\prime 3}}{12}\right)\right] \exp \left[\mathrm{i}\left(\frac{r^{2}}{4 \tau^{\prime \prime}}-\frac{\mathcal{F} \cdot \boldsymbol{r} \tau^{\prime \prime}}{2}-\frac{\mathcal{F}^{2} \tau^{\prime \prime 3}}{12}\right)\right] \\
=\left(\frac{4 \pi \tau^{\prime} \tau^{\prime \prime}}{\left(\tau^{\prime \prime}-\tau^{\prime}\right) \mathrm{i}}\right)^{3 / 2} \exp \left[-\mathrm{i} \frac{1}{12} \mathcal{F}^{2}\left(\tau^{\prime \prime}-\tau^{\prime}\right)^{3}\right] .
\end{gathered}
$$

The integrations over $\tau^{\prime}$ and $\tau^{\prime \prime}$ in terms of Airy functions (cf appendix B) lead to the following result for the normalization factor $f_{0}$ :

$$
f_{0}^{2}=\left[\tilde{f}_{0}\right]^{*} f_{0}=\frac{1}{2 \pi L(\mathcal{F})}
$$

where

$$
L(\mathcal{F})=2 \pi \mathcal{F}^{-1 / 3} I\left(-E \mathcal{F}^{-2 / 3}\right) .
$$

For $\mathcal{F} \rightarrow 0$, the energy in the static field behaves as $E \rightarrow-1$, and consequently $L(\mathcal{F}) \rightarrow 1$ ( $\operatorname{cf}(\mathrm{C} 3)$ ). Thus, for zero static field, $f_{0}$ coincides with the normalization factor $N=1 / \sqrt{2 \pi}$ of the bound state (17). Note that results (95) and (96) for the static field also follow from general results (89) or (90): for this case, $\phi_{n}, f_{n} \propto \delta_{n, 0}, K_{0}=\bar{K}_{0}=1$, and the integral over $\tau$ gives the function $I\left(-E \mathcal{F}^{-2 / 3}\right)$.

The above results of direct calculations for the normalized functions $\Phi_{E}(\boldsymbol{r})$ and $\tilde{\Phi}_{E}(\boldsymbol{r})$ agree with the formal definition (78). Indeed, the Green function of an electron in the $\delta$ potential plus a static electric field may be presented as follows [3]:

$$
\mathcal{G}_{\mathcal{E}}\left(\boldsymbol{r}, \boldsymbol{r}^{\prime}\right)=G_{\mathcal{E}}\left(\boldsymbol{r}, \boldsymbol{r}^{\prime}\right)+4 \pi \frac{G_{\mathcal{E}}(\boldsymbol{r}, 0) G_{\mathcal{E}}\left(\boldsymbol{r}^{\prime}, 0\right)}{1-g_{\mathcal{F}}(\mathcal{E})}
$$

where $g_{\mathcal{F}}(\mathcal{E})$ is the regularized function (35). Obviously, the equation for the (complex) resonance energy $\mathcal{E}=E, 1-g_{\mathcal{F}}(E)=0$, coincides with (47). Taking into account the relation

$$
\left.\left[1-g_{\mathcal{F}}(\mathcal{E})\right]\right|_{\mathcal{E} \rightarrow E} \longrightarrow \frac{1}{2} L(\mathcal{F})(\mathcal{E}-E)
$$

and comparing the residue of $\mathcal{G}_{\mathcal{E}}$ at $\mathcal{E}=E$ with (78), we obtain again the results (77), (93) and (95). It is interesting to note that for the QS state in a static electric field the three-dimensional relation $\tilde{\Phi}_{E}(\boldsymbol{r})=\left[\Phi_{E}(\boldsymbol{r})\right]^{*}$ is satisfied, which is similar to that for the one-dimensional case of QS states in a central potential.

The factor $f_{0}$ in (95) thus proves to be complex, but this complexity is not surprising for resonance states in collisional problems (see, e.g., equations (17) and (31) in [161]) and arises as a result of an analytical continuation procedure for the normalization. The results (72) and (93) demonstrate that the integral representations for $\Phi_{E}$ and $\tilde{\Phi}_{E}$ are very similar, differing only in their integration limits, i.e. $\tau^{\prime} \in[0, \infty)$ and $\tau^{\prime \prime} \in(-\infty, 0]$. However, the principal difference is this: in the calculation of the double integral (92) over $\tau^{\prime}$ and $\tau^{\prime \prime}$, one has a singularity in the integrand only at the isolated point $\tau^{\prime}=\tau^{\prime \prime}=0$ of the $\tau^{\prime}, \tau^{\prime \prime}$ plane (see (94)), 
where the intervals of $\tau^{\prime}$ and $\tau^{\prime \prime}$ overlap, and such a singularity is regularizable. In contrast, in calculating the standard normalization integral for $\Phi_{\epsilon}$ one has a singularity along the whole line $\tau^{\prime}=\tau^{\prime \prime}$ (since now both arguments run over the interval $[0, \infty]$ ) and thus the integral is non-regularizable. Obviously, such a situation also occurs in the calculation of matrix elements of $\boldsymbol{r}$ (see (99) and (100) below). Without using a proper dual bra vector, $\left\langle\tilde{\Phi}_{\epsilon}\right|$, instead of the bra vector $\left\langle\Phi_{\epsilon}\right|$, similar divergences can arise in calculations of matrix elements with higher-order PT wavefunctions for QQES, as was demonstrated in [169]. For this case the expression for $\left|\Phi_{\epsilon}\right\rangle$ involves the (retarded) atomic Green functions, $G_{\epsilon}^{a}$, in (8) having singularities of the form $1 /(x+\mathrm{i} 0)$ in the integrals over continuum states. For $\left\langle\Phi_{\epsilon}\right|$, these singularities transform to $1 /(x-\mathrm{i} 0)$, and non-integrable singularities $1 /\left[(x+\mathrm{i} 0)\left(x^{\prime}-\mathrm{i} 0\right)\right]$ at $x \rightarrow x^{\prime}$ arise in the resulting expressions for matrix elements of physical quantities. Using $\left\langle\tilde{\Phi}_{\epsilon}\right|$ as the bra vector, we deal only with singularities of the form $1 /(x+\mathrm{i} 0)^{n}$ in matrix elements, and such singularities can be regularized in standard ways (see, e.g., [105]). We note that in equations (10) and (11) (for an initial bound state $\left.\left|\psi_{n}\right\rangle\right)$ there are no bra vectors $\left\langle\Phi_{\epsilon}\right|$, so in calculations of the complex quasienergy $\epsilon$ a normalization procedure is not necessary.

\subsection{Perturbative calculation of $\Delta \epsilon^{\mathrm{opt}}$ with quasistationary basis functions}

Returning to the calculation of $\Delta \epsilon^{\text {opt }}$, we note that, with the above-defined normalization, the starting formula for $\Delta \epsilon^{\mathrm{opt}} \equiv \epsilon-E$ differs from (11) only by using the dual bra state, $\left\langle\tilde{\Phi}_{E}\right|$, instead of $\left\langle\psi_{n}\right| \equiv\left\langle\Phi_{E}\right|$ :

$\Delta \epsilon^{\mathrm{opt}}=\left\langle\left\langle\tilde{\Phi}_{E}\left|V_{L}(\boldsymbol{r}, t)\right| \Phi_{\epsilon}\right\rangle\right\rangle=\frac{1}{2} F\left(\left\langle\tilde{\Phi}_{E}|\boldsymbol{e} \cdot \boldsymbol{r}| \Phi^{+1}\right\rangle+\left\langle\tilde{\Phi}_{E}\left|\boldsymbol{e}^{*} \cdot \boldsymbol{r}\right| \Phi^{-1}\right\rangle\right)$.

The integration over $\boldsymbol{r}$ in (98) with functions (75) and (93) is carried out using the following relations:

$$
\begin{gathered}
\int \mathrm{d} \boldsymbol{r} \exp \left[-\mathrm{i}\left(\frac{r^{2}}{4 \tau^{\prime}}-\frac{\mathcal{F} \cdot \boldsymbol{r} \tau^{\prime}}{2}-\frac{\mathcal{F}^{2} \tau^{\prime 3}}{12}\right)\right] \boldsymbol{r} \exp \left[\mathrm{i}\left(\frac{r^{2}}{4 \tau^{\prime \prime}}-\frac{\mathcal{F} \cdot \boldsymbol{r} \tau^{\prime \prime}}{2}-\frac{\mathcal{F}^{2} \tau^{\prime \prime 3}}{12}\right)\right] \\
=-\mathcal{F} \tau^{\prime} \tau^{\prime \prime}\left(\frac{4 \pi \tau^{\prime} \tau^{\prime \prime}}{\left(\tau^{\prime \prime}-\tau^{\prime}\right) \mathrm{i}}\right)^{3 / 2} \exp \left[-\frac{1}{12} \mathrm{i} \mathcal{F}^{2}\left(\tau^{\prime \prime}-\tau^{\prime}\right)^{3}\right] \\
\int \mathrm{d} \boldsymbol{r} \exp \left[\mathrm{i}\left(\frac{r^{2}}{4 \tau^{\prime}}-\frac{\mathcal{F} \cdot \boldsymbol{r} \tau^{\prime}}{2}-\frac{\mathcal{F}^{2} \tau^{\prime 3}}{12}\right)\right] \boldsymbol{r}(\boldsymbol{r} \cdot \boldsymbol{e}) \exp \left[\mathrm{i}\left(\frac{r^{2}}{4 \tau^{\prime \prime}}-\frac{\mathcal{F} \cdot \boldsymbol{r} \tau^{\prime \prime}}{2}-\frac{\mathcal{F}^{2} \tau^{\prime \prime} 3}{12}\right)\right] \\
=\left[\boldsymbol{e} \frac{\pi^{3 / 2}}{2}\left(\frac{4 \tau^{\prime} \tau^{\prime \prime}}{\left(\tau^{\prime \prime}-\tau^{\prime}\right) \mathrm{i}}\right)^{5 / 2}+\mathcal{F}(\mathcal{F} \cdot \boldsymbol{e})\left(\tau^{\prime} \tau^{\prime \prime}\right)^{2}\left(\frac{4 \pi \tau^{\prime} \tau^{\prime \prime}}{\left(\tau^{\prime \prime}-\tau^{\prime}\right) \mathrm{i}}\right)^{3 / 2}\right] \\
\times \exp \left[-\frac{1}{12} \mathrm{i} \mathcal{F}^{2}\left(\tau^{\prime \prime}-\tau^{\prime}\right)^{3}\right] .
\end{gathered}
$$

The result for $\Delta \epsilon^{\mathrm{opt}}$ coincides exactly with that in (68), where the overall factor in the denominator of the second term on the right-hand side is proportional to the factor $L(\mathcal{F})$ in (96). After simple but tedious transformations, the result for $\Delta \epsilon^{\mathrm{opt}}$ can also be presented in a form that coincides with (65) by taking into account the explicit form of $\Sigma(-E)$ and the definition of $L(\mathcal{F})$. The final result is

$$
\begin{gathered}
\Delta \epsilon^{\mathrm{opt}}=\frac{F^{2}}{2 \omega^{2}}\left\{1-\frac{2 \pi \mathcal{F}}{3 \omega^{2} L(\mathcal{F})}\left[\sum_{n=-1}^{1}(-1)^{n} C_{2}^{n+1}\left(\xi_{n} J\left(\xi_{n}\right)+\frac{1}{8}\left(2+3 \Lambda^{2}\right) I^{\prime}\left(\xi_{n}\right)\right)\right.\right. \\
\left.\left.-\frac{3}{4} \pi \mathcal{F}^{1 / 3} \Lambda^{2} \sum_{n= \pm 1} \frac{\left(I(\xi)+I\left(\xi_{n}\right)\right)^{2}}{1+\pi \mathcal{F}^{1 / 3} J\left(\xi_{n}\right)}\right]\right\} .
\end{gathered}
$$


The coincidence of $\Delta \epsilon^{\mathrm{opt}}$ in (101) with the results of the independent calculations in section 5 justifies the utility of the normalization procedure used for the calculations with QQES wavefunctions (see also section 7 below). Finally, we note that the complexity of the QS wavefunction and of the normalization factor is not conventional and it is not often taken into account in calculations involving a static field. The error caused by this inaccuracy may be important for a strong field. For example, in the photodetachment calculations in [24] the authors used only the real part of $G_{E}(r, 0)$ in (77) and $\operatorname{Re} f_{0}^{2}$ (both for $E=E_{0}=-1$ ) in defining the wavefunction for the initial state and its normalization factor.

\section{The dipole moment and polarizability of a decaying atomic system}

Since $\Delta \epsilon^{\mathrm{opt}} \propto F^{2}$, for a careful analysis of $\mathcal{F}$ and its $\omega$ dependence it is convenient to write $\Delta \epsilon^{\mathrm{opt}}$ similarly to the Stark shift of a bound $S$-level in the weak laser field (see (110) below):

$$
\Delta \epsilon^{\mathrm{opt}}=-\frac{1}{4} \alpha(\mathcal{F} ; \omega) F^{2}
$$

where $\alpha(\mathcal{F} ; \omega)$ can be considered as a generalization of the ordinary dynamic polarizability $\alpha(\omega)$ to the case of a non-zero static field. Nevertheless, the notation 'dynamic polarizability' for $\alpha(\mathcal{F} ; \omega)$ requires some explanation since, as we demonstrate below, in strong fields the standard definition for the field-induced dipole moment fails and the term 'polarizability' has only a limited applicability.

\subsection{Connection between the polarizability and the dynamical Stark effect for a weak laser and zero static field}

For background, we discuss first the known results for the case of $\mathcal{F}=0$ and a weak laser field, when the dynamic polarizability tensor $\alpha_{i k}(\omega)$ determines the (linear in $F$ ) dipole moment of the atom induced by a laser field $\boldsymbol{F}(t)=F \operatorname{Re}\{e \exp (-\mathrm{i} \omega t)\}[170]$ :

$$
\boldsymbol{d}(t)=\frac{1}{2}\left(\boldsymbol{d}_{\omega} \mathrm{e}^{-\mathrm{i} \omega t}+\boldsymbol{d}_{-\omega} \mathrm{e}^{\mathrm{i} \omega t}\right) \quad \boldsymbol{d}_{-\omega}=\boldsymbol{d}_{\omega}^{*}
$$

where

$$
\left(\boldsymbol{d}_{\omega}\right)_{i}=\sum_{k=x, y, z} \alpha_{i k}(\omega)(e)_{k} F
$$

and where

$$
\alpha_{i k}(\omega)=\alpha(\omega) \delta_{i k} \quad \text { and } \quad d_{\omega}=\alpha(\omega) e F
$$

for S-states. An explicit expression for $\alpha_{i k}(\omega)$ may be obtained by calculating $\boldsymbol{d}_{\omega}$ as the expectation value of the dipole moment operator $\hat{\boldsymbol{d}}=-\boldsymbol{r}$ including corrections to the wavefunction of an initial state $\psi_{0}$ up to first order in $F$,

$$
\begin{aligned}
\boldsymbol{d}_{\omega} & =-2\left(\left\langle\Phi^{+1}|\boldsymbol{r}| \psi_{0}\right\rangle+\left\langle\psi_{0}|\boldsymbol{r}| \Phi^{-1}\right\rangle\right) \\
& =-\left\langle\psi_{0}\left|\left\{\boldsymbol{e} \cdot \boldsymbol{r} G_{E_{0}-\omega}^{*} \boldsymbol{r}+\boldsymbol{r} G_{E_{0}+\omega} \boldsymbol{e} \cdot \boldsymbol{r}\right\}\right| \psi_{0}\right\rangle F
\end{aligned}
$$

where $G_{E}=1 /\left(E-H_{0}+\mathrm{i} 0\right)$ is the (retarded) atomic Green function, and we assume that $\Phi^{ \pm 1}$ are the QQES harmonics in equation (71) for zero static field.

The connection between the frequency-dependent polarizability and the field-induced energy shift may be sketched as follows. On the one hand, we note that for real negative $E_{0}$ and positive $\omega$ the sign of the infinitesimal i0 in the definition of $G_{E}$ is inconsequential and we may change $G_{E_{0}-\omega}^{*}$ to $G_{E_{0}-\omega}$. Because of this, for an initial bound state, $\alpha_{i k}(\omega)$ coincides with the tensor describing elastic photon scattering by the atom (see [170] for more details). 
On the other hand, a direct PT calculation of the second order in $F$ correction, $\Delta \epsilon_{2}$, to $E_{0}$ in accordance with (11) also leads to an expression for $\Delta \epsilon_{2}$ in terms of the photon scattering amplitude:

$$
\Delta \epsilon_{2}=\frac{1}{4}\left\langle\psi_{0}\left|\left\{e \cdot r G_{E_{0}-\omega} e^{*} \cdot r+e^{*} \cdot r G_{E_{0}+\omega} e \cdot r\right\}\right| \psi_{0}\right\rangle F^{2} .
$$

Therefore, it is possible to connect $\Delta \epsilon_{2}$ with the polarizability, namely, substituting $G_{E_{0}-\omega}^{*} \rightarrow$ $G_{E_{0}-\omega}$ in equation (107), one obtains

$$
\Delta \epsilon_{2}=-\frac{1}{4} F e^{*} \cdot d_{\omega}=-\frac{1}{4} \sum_{i, k} \alpha_{i k}(\omega)\left(e^{*}\right)_{i}(e)_{k} F^{2}
$$

or

$$
\Delta \epsilon_{2}=-\frac{1}{4} \alpha(\omega) F^{2}
$$

for S-states. Thus, both the dynamic polarizability and the photon scattering amplitude may be used equivalently for the analysis of the laser-induced correction to the energy $E_{0}$ of a bound state. Moreover, the imaginary part of $\alpha(\omega)$, which is non-zero for $\omega>\left|E_{0}\right|$, determines the phase lag of the (real) dipole moment $d(t)$ in (103) or the ionization width of a bound state $\left|\psi_{0}\right\rangle$ in (110).

\subsection{The dual dipole moment and the nonlinear static polarizability in a strong static electric} field

To apply the above results to the case $\mathcal{F} \neq 0$ we observe that now we deal with the dipole moment and polarizability of an unstable quantum system (decaying in a static field). In view of the static field ionization it is evident physically that for such a system the dipole moment $\boldsymbol{d}(t)$ (i.e. the expectation value of $\boldsymbol{r}$ ) induced even by a weak laser field $\boldsymbol{F}(t)$ tends to infinity with increasing $\mathcal{F}$. A mathematical consequence of this fact is that the matrix elements in (106) diverge if we use for $\left\langle\psi_{0}\right|,\left\langle\Phi^{0}\right|$, or $\left\langle\Phi^{+1}\right|$ the standard (i.e. 'non-dual') expressions discussed in section 6.1. On the other hand, for the QQES or quasistationary (resonance) state it is possible to introduce the following formal definition of the ('dual') dipole moment (cf (86)):

$$
\tilde{\boldsymbol{d}}(t)=\left\langle\tilde{\Phi}_{\epsilon}(\boldsymbol{r}, t)|\hat{\boldsymbol{d}}| \Phi_{\epsilon}(\boldsymbol{r}, t)\right\rangle
$$

which is finite for strong fields $\mathcal{F}$ and/or $\boldsymbol{F}(t)$. Obviously, $\tilde{\boldsymbol{d}}(t)$ is complex and is not completely identical to the ordinary dipole moment $\boldsymbol{d}(t)$. Nevertheless, $\tilde{\boldsymbol{d}}(t)$ is connected with the complex (quasi)energy by means of the generalized (for QQES) Hellmann-Feynman theorem similarly to the case of a bound level in a static field (see, e.g., section 76 in [90]).

As an illustration we consider firstly the case of a strong static field and zero laser field. Employing the formalism of dual functions, the Hellmann-Feynman theorem for the QS state $\Phi_{E}(r)$ with complex $E(\lambda)$ ( $\lambda$ is a parameter of the Hamiltonian $H$ ) permits us to obtain the following relation:

$$
\frac{\partial E(\lambda)}{\partial \lambda}=\left\langle\tilde{\Phi}_{E}(\boldsymbol{r})\left|\frac{\partial H(\boldsymbol{r}, \lambda)}{\partial \lambda}\right| \Phi_{E}(\boldsymbol{r})\right\rangle
$$

which is similar to that for the case of bound states, taking into account the relation $\left\langle\tilde{\Phi}_{E}\right|(H-E)=0$. Considering $\lambda=\mathcal{F}$, we obtain

$$
-\frac{\partial E}{\partial \mathcal{F}}=-\frac{\partial E}{\partial \mathcal{F}} \frac{\mathcal{F}}{\mathcal{F}}=\left\langle\tilde{\Phi}_{E}(\boldsymbol{r})|\hat{\boldsymbol{d}}| \Phi_{E}(\boldsymbol{r})\right\rangle=\tilde{\boldsymbol{d}}_{0}
$$


in accordance with (111). For the $\delta$-model, the matrix element of $\hat{\boldsymbol{d}}=-\boldsymbol{r}$ in (113) is easily calculated. Using equations (72) and (93) for $\Phi_{E}$ and $\tilde{\Phi}_{E}$, we perform the spatial integration first, employing the integral given in (99). The matrix element is then reduced to the integral

$$
\lambda=\int_{0}^{\infty} \mathrm{d} \tau^{\prime} \int_{-\infty}^{0} \mathrm{~d} \tau^{\prime \prime} \frac{\tau^{\prime} \tau^{\prime \prime}}{\left(\tau^{\prime \prime}-\tau^{\prime}\right)^{3 / 2}} \exp \left[-\mathrm{i} \frac{1}{12} \mathcal{F}^{2}\left(\tau^{\prime \prime}-\tau^{\prime}\right)^{3}\right]
$$

Using the change of variables,

$$
\tau^{\prime \prime}-\tau^{\prime}=s \quad \tau^{\prime \prime}+\tau^{\prime}=p
$$

we reduce this integral to

$$
\lambda=\frac{1}{4} \int_{-\infty}^{0} \frac{\mathrm{d} s}{s^{3 / 2}} \exp \left[-\mathrm{i} \frac{1}{12} \mathcal{F}^{2} s^{3}\right] \int_{-s}^{s} \mathrm{~d} p\left(p^{2}-s^{2}\right)
$$

which can be expressed in terms of the Green function of the electron in a static field and its derivatives according to equation (B2) and the equations for $I(\xi)$ in appendix C. The final result for $\tilde{\boldsymbol{d}}_{0}$ is

$$
\tilde{\boldsymbol{d}}_{0}=\alpha(\mathcal{F}) \mathcal{F} \quad \alpha(\mathcal{F}) \equiv-\frac{1}{\mathcal{F}} \frac{\partial E}{\partial \mathcal{F}}=\frac{I^{\prime \prime}(\xi)}{6 I(\xi) \mathcal{F}^{4 / 3}}
$$

where $I(\xi)$ is defined by (50) and where

$$
\xi=-E / \mathcal{F}^{2 / 3} \text {. }
$$

The nonlinear polarizability $\alpha(\mathcal{F})$ in $(114)$ may be used for the calculation of the energy $E$ by integration of the differential equation $\partial E / \partial \mathcal{F}=-\mathcal{F} \alpha(\mathcal{F})$ with the boundary condition $\lim _{\mathcal{F} \rightarrow 0} E=E_{0}=-1$. For the integration it is convenient to use instead of $E(\mathcal{F})$ the new function, $\xi=\xi(\mathcal{F})$, defined in (115), and the following relations: $I(\xi)=-J^{\prime}(\xi)$, $I^{\prime \prime}(\xi)=2 J(\xi)+4 \xi I(\xi)$. The resulting equation for $\xi(\mathcal{F})$,

$$
\frac{\partial \xi}{\partial \mathcal{F}}=-\frac{1}{3} \frac{J(\xi)}{\mathcal{F} J^{\prime}(\xi)}
$$

is integrated immediately:

$$
\pi \mathcal{F}^{1 / 3} J\left(-E / \mathcal{F}^{2 / 3}\right)+1=0 .
$$

This result coincides with the exact expression (47) for the complex energy in a static field. Alternatively, calculating the derivative $\partial E / \partial \mathcal{F}$ in (113) using the transcendental equation (47) for $E(\mathcal{F})$, we obtain the expression $(114)$ for $\alpha(\mathcal{F})$ without an explicit calculation of $\tilde{\boldsymbol{d}}_{0}$. In the limit $\mathcal{F} \rightarrow 0$, for which $E=-1$, the asymptotic result for $\alpha(\mathcal{F})$ in (114) is

$$
\alpha(\mathcal{F} \rightarrow 0)=\frac{1}{8}+\frac{\mathrm{i}}{3 \mathcal{F}^{2}} \mathrm{e}^{-4 /(3 \mathcal{F})} .
$$

This expression coincides with the independent calculation of $\alpha(\mathcal{F} \rightarrow 0)$ as $\alpha(\mathcal{F})=$ $(-1 / \mathcal{F}) \partial E / \partial \mathcal{F}$ using for $E$ the weak-field result (48). Neglecting the imaginary part, equation (117) for $\alpha(\mathcal{F} \rightarrow 0)$ coincides with the well known PT result for the static polarizability $\alpha^{p t}$ for a weakly bound level (see, for example, section 76 in [90]),

$$
\alpha^{p t}=\frac{1}{8} \quad\left(=\frac{m e^{2}}{4 \hbar^{2} \kappa^{4}} \quad \text { in absolute units }\right) .
$$

This example demonstrates the self-consistency of our definitions of the dipole moment and polarizability for quasistationary states as well as a complicated, nonlinear relation between the polarizability of a level and its complex energy in a strong field. 
7.3. The dual dipole moment and the dynamic polarizability for weak laser and strong static fields

Returning to the case $\boldsymbol{F}(t) \neq 0$, we present $\tilde{\boldsymbol{d}}(t)$ in (111) as the Fourier expansion (cf (103)),

$$
\tilde{\boldsymbol{d}}(t)=\frac{1}{2} \sum_{s=-\infty}^{\infty} \tilde{\boldsymbol{d}}_{s \omega} \mathrm{e}^{-\mathrm{i} s \omega t}
$$

where $\tilde{\boldsymbol{d}}_{s \omega}(\xi)=\tilde{\boldsymbol{d}}_{-s \omega}(-\xi)$. The Fourier components $\tilde{\boldsymbol{d}}_{s \omega}$ with $s= \pm 1$ are connected with the complex quasienergy $\epsilon$ by means of the Hellmann-Feynman theorem, which can be derived for the QQES in the following form (cf with the result for stationary QES in $[63,64]$ ):

$$
\frac{\partial \epsilon(\lambda)}{\partial \lambda}=\left\langle\left\langle\tilde{\Phi}_{\epsilon}(\boldsymbol{r}, t)\left|\frac{\partial H(\lambda)}{\partial \lambda}\right| \Phi_{\epsilon}(\boldsymbol{r}, t)\right\rangle .\right.
$$

Indeed, taking into account (70) and assuming $\lambda=F$ one obtains

$$
\frac{\partial \epsilon}{\partial F}=-\frac{1}{4}\left(e^{*} \cdot \tilde{\boldsymbol{d}}_{\omega}+e \cdot \tilde{\boldsymbol{d}}_{-\omega}\right)
$$

where

$$
\tilde{\boldsymbol{d}}_{ \pm \omega}=2\left\langle\left\langle\tilde{\Phi}_{\epsilon}(\boldsymbol{r}, t)\left|\hat{\boldsymbol{d}} \mathrm{e}^{ \pm \mathrm{i} \omega t}\right| \Phi_{\epsilon}(\boldsymbol{r}, t)\right\rangle\right\rangle .
$$

These results are valid for an arbitrary $F$ (and for $\mathcal{F} \neq 0$ ).

To calculate $\Delta \epsilon^{\text {opt }}$ we confine ourselves to the case of weak $F$, but assume that $\mathcal{F}$ may be strong. Concerning the vector structure of $\tilde{\boldsymbol{d}}_{ \pm \omega}$, we note that the relations (105) are no longer valid even for the initial S-state (17) since, in view of the static-field-induced anisotropy of an atom (or an ion), the polarizability is a tensor with two independent components. Using symmetry arguments, the vector $\tilde{\boldsymbol{d}}_{\omega}$ can be presented instead in terms of two partial polarizabilities, $\alpha_{0}(\mathcal{F} ; \omega)$ and $\alpha_{1}(\mathcal{F} ; \omega)$, which are invariant with respect to the polarization state of the laser field and the geometry of the fields:

$$
\tilde{\boldsymbol{d}}_{\omega}=\left[\alpha_{0}(\mathcal{F} ; \omega) \boldsymbol{e}+\alpha_{1}(\mathcal{F} ; \omega)\left(\boldsymbol{e} \cdot \boldsymbol{e}_{0}\right) \boldsymbol{e}_{0}\right] F
$$

where $e_{0}=\mathcal{F} / \mathcal{F}$. The result for $\tilde{\boldsymbol{d}}_{-\omega}$ follows from $\tilde{\boldsymbol{d}}_{\omega}$ by substituting $e \rightarrow e^{*}$, so that

$$
e^{*} \cdot \tilde{\boldsymbol{d}}_{\omega}=e \cdot \tilde{\boldsymbol{d}}_{-\omega} .
$$

To calculate invariant polarizabilities $\alpha_{0}$ and $\alpha_{1}$, we present $\tilde{\boldsymbol{d}}_{\omega}$ in equation (122) to first order in $F$ as (cf (106))

$$
\tilde{\boldsymbol{d}}_{\omega}=-2\left(\left\langle\tilde{\Phi}^{+1}|\boldsymbol{r}| \Phi_{E}\right\rangle+\left\langle\tilde{\Phi}_{E}|\boldsymbol{r}| \Phi^{-1}\right\rangle\right) .
$$

The matrix elements in this equation are calculated similarly to the calculation of $\Delta \epsilon^{\mathrm{opt}}$ in (98), resulting in (101). The result for $\boldsymbol{d}_{\omega}$ may be presented in the form (123), where

$$
\alpha_{0}=-\frac{2}{\omega^{2}}+\frac{4 \pi \mathcal{F}}{3 \omega^{4} L(\mathcal{F})} \sum_{n=-1}^{1}(-1)^{n} C_{2}^{n+1}\left[\xi_{n} J\left(\xi_{n}\right)+\frac{1}{4} I^{\prime}\left(\xi_{n}\right)\right]
$$

and

$\alpha_{1}=-\frac{\pi \mathcal{F}}{\omega^{4} L(\mathcal{F})}\left[\sum_{n=-1}^{1} C_{2}^{n+1} I^{\prime}\left(\xi_{n}\right)+\pi \mathcal{F}^{1 / 3}\left(\frac{\left(I(\xi)+I\left(\xi_{+1}\right)\right)^{2}}{1+\pi \mathcal{F}^{1 / 3} J\left(\xi_{+1}\right)}+\frac{\left(I(\xi)+I\left(\xi_{-1}\right)\right)^{2}}{1+\pi \mathcal{F}^{1 / 3} J\left(\xi_{-1}\right)}\right)\right]$. 
To confirm these results, obtained using (125), we express $\tilde{\boldsymbol{d}}_{\omega}$ in terms of the Green function (97) (which takes account of the atomic and static fields only) (cf (107)):

$$
\tilde{\boldsymbol{d}}_{\omega}=-\left\langle\tilde{\Phi}_{E}\left|\left\{\boldsymbol{e} \cdot \boldsymbol{r} \mathcal{G}_{E-\omega} \boldsymbol{r}+\boldsymbol{r} \mathcal{G}_{E+\omega} e \cdot r\right\}\right| \Phi_{E}\right\rangle F
$$

where the bra and ket states are given by (72) and (93), respectively. Using the explicit form (97) for $\mathcal{G}_{\mathcal{E}}$ in terms of $G_{\mathcal{E}}$ and the Fourier transform of the time-dependent Green function (B2) for $G_{\mathcal{E}}$, one obtains by direct calculation the results (123), (126) and (127) for $\tilde{\boldsymbol{d}}_{\omega}$ once again.

From the identity (124), the expression (121) for $\Delta \epsilon^{\mathrm{opt}}$ can be written as

$$
\frac{\partial \Delta \epsilon^{\mathrm{opt}}}{\partial F}=-\frac{1}{2} e^{*} \cdot \tilde{\boldsymbol{d}}_{\omega}
$$

and, after the trivial integration over $F$, taking account of (123) we obtain (102), where

$$
\alpha(\mathcal{F} ; \omega)=\alpha_{0}(\mathcal{F} ; \omega)+\frac{1}{2} \Lambda^{2} \alpha_{1}(\mathcal{F} ; \omega)
$$

where $\Lambda^{2}=2\left|e \cdot e_{0}\right|^{2}(\operatorname{cf}(20))$. The results (102), (126), (127) and (130) coincide exactly with the independent, direct calculation of $\Delta \epsilon^{\mathrm{opt}}$ in (98) and (101). Thus, we consider $\alpha(\mathcal{F} ; \omega)$ as the combined polarizability in (130) and this quantity describes entirely the dynamical Stark effect for a static-field-distorted atomic level as well as (one-photon) photodetachment (section 9). Nevertheless, a separate analysis of the components $\alpha_{0}$ and $\alpha_{1}$ may be important for other physical processes involving the static-field-distorted atomic objects, in particular, the asymptotic (van der Waals) interaction or light scattering by atoms in the presence of a static field. In the last case, a strong static field induces a number of specific, photon polarization effects which are caused by imaginary (i.e. skew-Hermitian) parts of $\alpha_{0}$ and $\alpha_{1}$, which are discussed briefly below.

\subsection{Static-electric-field-induced polarization anomalies in light scattering by atoms and ions}

Obviously, the definitions of $\alpha_{0,1}(\mathcal{F} ; \omega)$ in equations (123) and (128) are quite general and are valid for any system (having a non-degenerate, spherically symmetric initial state) in the presence of a static electric field. Moreover, the matrix element (128) is connected with the (elastic) scattering amplitude of the incident photon having polarization vector $\boldsymbol{e}$. Thus, the differential cross section for the emission of a spontaneous photon in the direction $\hat{k}^{\prime}$ and with the (detected) polarization $e^{\prime}$ may be obtained similarly to the case of ordinary (i.e. without a static electric field) Rayleigh scattering (see, e.g., [170]):

$$
\begin{gathered}
\frac{\mathrm{d} \sigma^{\mathrm{sc}}}{\mathrm{d} \Omega_{\hat{k}^{\prime}}}=\frac{1}{4} r_{0}^{2} \omega^{4}\left|\alpha_{0}(\mathcal{F} ; \omega)\left(e \cdot e^{\prime *}\right)+\alpha_{1}(\mathcal{F} ; \omega)\left(e \cdot e_{0}\right)\left(e_{0} \cdot e^{\prime *}\right)\right|^{2} \\
=\frac{1}{4} r_{0}^{2} \omega^{4}\left[\left|\alpha_{0}\right|^{2}\left|e \cdot e^{\prime *}\right|^{2}+\left|\alpha_{1}\right|^{2}\left|\left(e \cdot e_{0}\right)\left(e_{0} \cdot e^{\prime *}\right)\right|^{2}\right. \\
\left.+2 \operatorname{Re}\left(\alpha_{0} \alpha_{1}^{*}\right) \operatorname{Re} A-2 \operatorname{Im}\left(\alpha_{0} \alpha_{1}^{*}\right) \operatorname{Im} A\right]
\end{gathered}
$$

where $A=\left(e \cdot e^{\prime *}\right)\left(e^{*} \cdot e_{0}\right)\left(e_{0} \cdot e^{\prime}\right)$, and $r_{0}=e^{2} / m c^{2} \simeq 2.82 \times 10^{-13} \mathrm{~cm}$ is the classical electron radius. For elliptically polarized photons, the explicit form of the real and imaginary parts of the combination of vectors $A$ in terms of real quantities is very complicated and is not reproduced here (see [171] for details of such calculations). We note only that $\operatorname{Im} A$ may be expressed as a sum of two terms proportional to $\xi$ and $\xi^{\prime}$, respectively, i.e. to the circular 
polarization degrees (or Stokes parameters $\xi_{2}$ and $\xi_{2}^{\prime}$ ) of the incident and scattered (detected) photons. In particular, for the case of a linearly polarized detected photon one obtains

$$
2 \operatorname{Im} A=\xi\left(e_{0} \cdot e^{\prime}\right)\left(\hat{k} \cdot\left[e^{\prime} \times e_{0}\right]\right)
$$

and for the case of a linearly polarized incident photon one obtains

$$
2 \operatorname{Im} A=\xi^{\prime}\left(e_{0} \cdot e\right)\left(\hat{k}^{\prime} \cdot\left[e_{0} \times e\right]\right) .
$$

The terms (133) and (134) describe specific, static-electric-field-induced polarization effects in the scattering of light by atoms. The term (133) describes circular dichroism (CD) in photon scattering: the number of detected photons with linear polarization is different for right and left helicities of a circularly (or elliptically) polarized incident laser beam. The term (134) describes the inverse effect, the appearance of an elliptical polarization of scattered radiation, i.e. the difference between the numbers of detected photons with right and left circular polarizations, for a linearly polarized incident laser beam. The intrinsic degree of circular polarization of the scattered photon, $\xi_{2}^{\text {sc }}$, may be derived in a standard way (see, e.g., section 48 in [170]) using the explicit form (131) for the differential cross section. For the most important case, $\hat{k} \cdot e_{0}=0$, in which the static field is orthogonal to the direction of a (linearly polarized) laser beam, one obtains

$$
\xi_{2}^{\mathrm{sc}}=-\frac{\sin 2 \phi\left(\hat{k} \cdot \hat{k}^{\prime}\right) \operatorname{Im}\left(\alpha_{0} \alpha_{1}^{*}\right)}{\left|\alpha_{0}\left[\hat{k}^{\prime} \times \hat{\epsilon}\right]+\alpha_{1} \cos \phi\left[\hat{k}^{\prime} \times e_{0}\right]\right|^{2}} .
$$

It is easy to show that for forward scattering, $\boldsymbol{k}^{\prime} \| \boldsymbol{k}, \xi_{2}^{\text {sc }}$ attains its maximum value,

$$
\left.\xi_{2}^{\mathrm{sc}}\right|_{\max }=-\frac{\operatorname{Im}\left(\alpha_{0} \alpha_{1}^{*}\right)}{\left|\alpha_{0}\right|\left|\alpha_{0}+\alpha_{1}\right|}
$$

at the angle $\phi=\phi_{\max }$, where $\tan \phi_{\max }=1+\left|\alpha_{1}\right| /\left|\alpha_{0}\right|$. For example, for the case in which $\mathcal{F}=0.06$ and $\omega=1.3$, numerical calculations of $\alpha_{0}$ and $\alpha_{1}$ for a $\delta$-model potential give $\phi_{\max }=66.313^{\circ}$ and $\left.\xi_{2}^{\mathrm{sc}}\right|_{\max }=0.238$. The maximum value of the circular dichroism term in (131) corresponds to the angle $\pi / 4$ between the vector $\mathcal{F}$ and the polarization vector $\boldsymbol{e}_{l}^{\prime}$ of the linearly polarized, scattered photon. Both effects vanish for $\mathcal{F} \| e_{l}$ and/or $\mathcal{F} \perp \hat{\epsilon}$, as well as for the total cross section, $\sigma^{\text {sc }}$, summed over the polarization states and integrated over directions of the scattered photon:

$$
\sigma^{\mathrm{sc}}=\frac{2}{3} \pi r_{0}^{2} \omega^{4}\left[\left|\alpha_{0}+\alpha_{1}\right|^{2}\left|\boldsymbol{e} \cdot \boldsymbol{e}_{0}\right|^{2}+\left|\alpha_{0}\right|^{2}\left(1-\left|\boldsymbol{e} \cdot \boldsymbol{e}_{0}\right|^{2}\right)\right] .
$$

Thus, $\alpha_{\|} \equiv \alpha_{0}+\alpha_{1}$ and $\alpha_{\perp} \equiv \alpha_{0}$ have the sense of 'parallel' and 'perpendicular' (to the static electric field $\mathcal{F}$ ) components of the dynamic polarizability tensor in the presence of a static electric field.

Previously, dichroic effects in atomic photoprocesses with unpolarized targets were predicted for some special situations. For example, circular dichroism in light scattering by atoms appears in resonant scattering involving a dipole-forbidden, quadrupole transition resonance [172]. Dichroism caused by static-field-induced resonances was discussed recently for two-photon transitions between bound levels with opposite parities [173] and also for the total photoelectron yield in combined, one- and two-photon ionization [174]. As shown above, a strong static electric field induces dichroic effects in ordinary, dipole Rayleigh or Raman scattering. Both the magnitude of the circular dichroism and the circular polarization degree of the scattered photon, $\xi_{2}^{\text {sc }}$, are determined by the same atomic parameter, $\operatorname{Im}\left(\alpha_{0} \alpha_{1}^{*}\right)$, which vanishes for real $\alpha_{0}$ and $\alpha_{1}$, i.e. neglecting the ionization of atoms in static and laser fields. Thus, both effects are 'ionization-induced' effects caused by an interference between 
real and imaginary parts of the scattering amplitude. That confirms the general statement of [175] on a 'dissipation-induced' origin of dichroic effects in atomic photoprocesses with unpolarized targets. Evidently, for weak $\mathcal{F}$ the dichroic effects are small since $\alpha_{1}(\mathcal{F} ; \omega) \sim \mathcal{F}^{2}$ for $\mathcal{F} \rightarrow 0$ and the term with $\left|\alpha_{0}\right|^{2}$ in equation (131) is dominant. Figures 2 and 3 demonstrate the frequency dependence of the cross section (131) and the dichroic ratio, $\delta=\left(\mathrm{d} \sigma^{+}-\mathrm{d} \sigma^{-}\right) /\left(\mathrm{d} \sigma^{+}+\mathrm{d} \sigma^{-}\right)$, for the forward scattering of right $(+)$and left $(-)$circularly polarized incident photons by $\mathrm{H}^{-}$in the presence of an orthogonal static electric field, $\mathcal{F} \perp \hat{k}$, with $\mathcal{F}=0.06$ and 0.15 . The linear polarization of the scattered (detected) photon is assumed to be measured at the angle $\pi / 4$ with respect to $\mathcal{F}$. It is seen that the dichroic effects are most important for near-threshold frequencies $\omega$ and have a considerable magnitude for the static fields considered. Note, that for higher frequencies, $\omega \gg 1$, we have $\alpha_{0}(\mathcal{F}, \omega) \approx-2 / \omega^{2}$ and $\alpha_{1}(\mathcal{F}, \omega) \propto \omega^{-4}$, and thus $\mathrm{d} \sigma^{\text {sc }} / \mathrm{d} \Omega_{\hat{k}^{\prime}}$ in (131) tends to the classical, 'Thomson' limit, $r_{0}^{2}\left|e \cdot e^{\prime *}\right|^{2}$.

\subsection{Summary and observations}

We have presented above three independent derivations for $\Delta \epsilon^{\text {opt }}$ based on: (a) the exact result for $\epsilon=\epsilon(F, \mathcal{F})$ in the QQES approach (see (65) or (68)); (b) the perturbation theory result for $\epsilon$ in a basis of (properly normalized) QS states in a static electric field (equation (101)), and (c) the Hellmann-Feynman theorem for QQES, i.e. the relation between $\epsilon$ and the laserfield-induced dipole moment of the decaying system ((102) and (130)). Although differing in form, the final result is the same in all three cases. Nevertheless, these derivations are profoundly useful due to their elucidation of some general problems in the theory of atoms in strong laser fields, such as the normalization procedure for QQES and the concept of the polarizability and dipole moment for a decaying system. In particular, we emphasize that the terms 'polarizability' and 'dipole moment' for this case have a conditional sense, since in strong fields these quantities are connected with the amplitude of photon scattering rather than with the field-induced distortion of the charge density (as in the classical interpretation for the ordinary polarizability and the dipole moment $d(t)$ of a stable system in a weak field). Indeed, considering (instead of (128)) the standard expression (107) for the dipole moment as the definition of the dipole moment for the QS state $\left|\psi_{0}\right\rangle \equiv\left|\Phi_{E}\right\rangle$, the transformation of equation (109) into (108) is impossible since $G_{E_{0}-\omega}^{*} \neq G_{E_{0}-\omega}$ for complex $E_{0}$. (Moreover, without the substitution $\left\langle\psi_{0}\right| \rightarrow\left\langle\tilde{\psi}_{0}\right|$, the result for $\boldsymbol{d}_{\omega}$ will be divergent.) On the other hand, with the use of (121) and (128), we arrive at a result similar to (108), where the second-order matrix element is proportional to the amplitude of (stimulated) photon scattering by the atom in a QS state, i.e. it does not involve complex-conjugated Green functions. Since the iterative expansion of $\epsilon_{n}$ in (11) (the Brillouin-Wigner series for $\epsilon_{n}$ ) involves only the retarded Green function, $G_{\epsilon_{n}}^{a}$, this statement holds generally and also applies for the case of a strong laser field in the absence of static fields. Specifically, the third-order correction in $F$ to the dipole moment $\boldsymbol{d}(t)$ in (103) (cf (105)) was calculated in [169] as

$$
\boldsymbol{d}_{\omega}^{(3)}=\alpha(\omega) e F+\left(\gamma_{1} e+\gamma_{2} e^{*}\right) F^{3} .
$$

As demonstrated in [169], the expressions for the hyperpolarizabilities $\gamma_{1,2}$ diverge for above-threshold frequencies, $\omega>\left|E_{0}\right|$, and thus the results for this case were calculated by differentiating the fourth order in $F$ correction to the quasienergy with respect to $F$. Equation (121) shows that this method is equivalent to the use of $\tilde{\boldsymbol{d}}_{\omega}^{(3)}$ instead of $\boldsymbol{d}_{\omega}^{(3)}$. For $\omega<\left|E_{0}\right|$, the results for $\boldsymbol{d}_{\omega}^{(3)}$ and $\tilde{\boldsymbol{d}}_{\omega}^{(3)}$ coincide. 

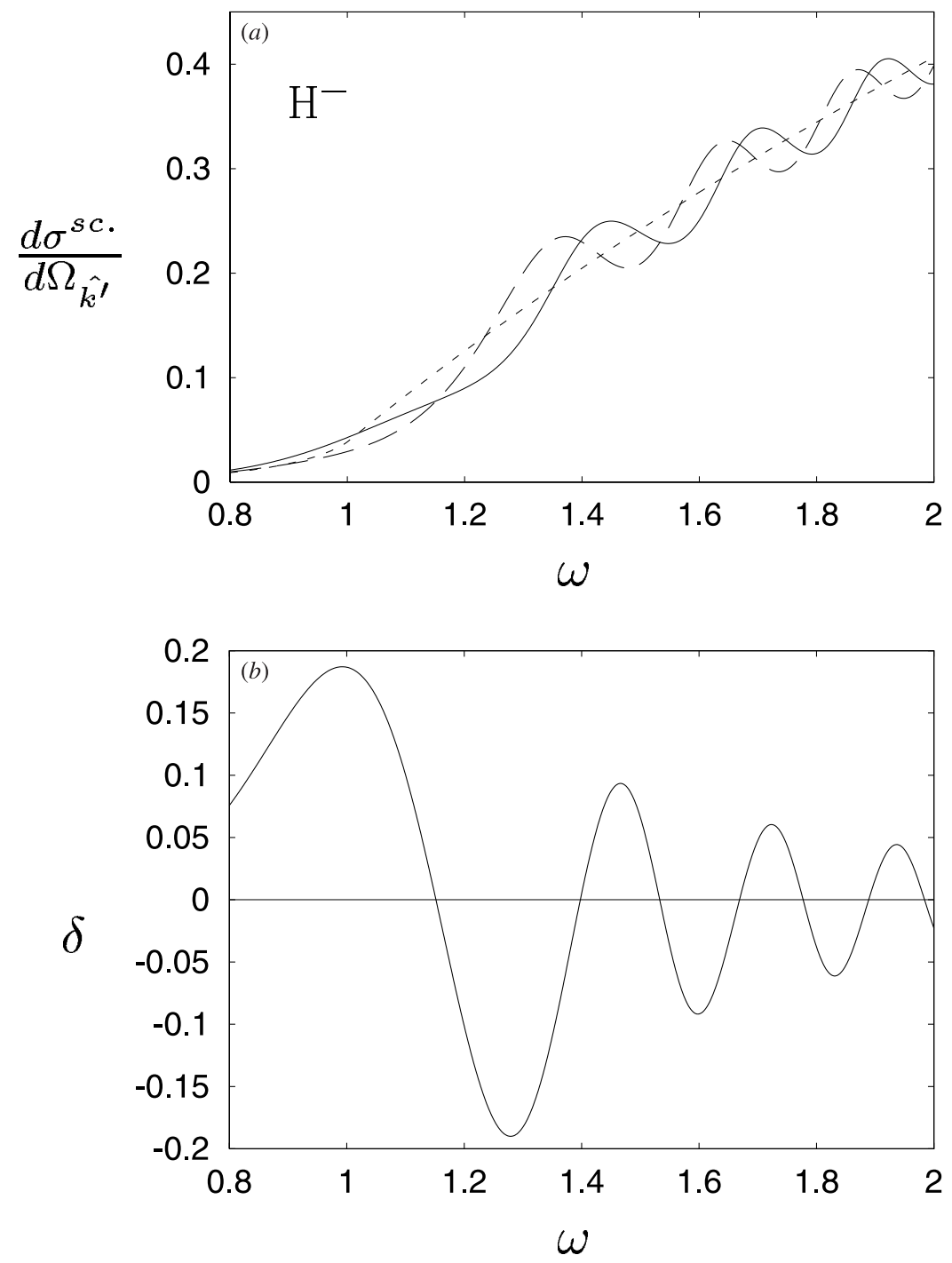

Figure 2. (a) Frequency dependence of the differential cross section (131), for the forward scattering of circularly polarized photons by an $\mathrm{H}^{-}$-ion at $\mathcal{F}=0.06$ (in units of $r_{0}^{2}$ ). The vector $\mathcal{F}$ is orthogonal to the incident photon beam, $\Lambda=1$. The polarization of the scattered (detected) photon is linear and oriented at the angle $\pi / 4$ with respect to $\mathcal{F}: e^{\prime} \cdot e_{0}=\cos (\pi / 4)$. The full (broken) curve corresponds to right, $\xi=+1$ (left, $\xi=-1$ ) helicity of incident photons. The dotted curve is the result for $\mathcal{F}=0$. (b) The dichroic ratio, $\delta=\left(\mathrm{d} \sigma^{+}-\mathrm{d} \sigma^{-}\right) /\left(\mathrm{d} \sigma^{+}+\mathrm{d} \sigma^{-}\right)$

Although we have analysed above only the response of the decaying system to the fundamental frequency $\omega$, the discussion of the relation between $\tilde{\boldsymbol{d}}(t)$ and the expectation value of $r$ is valid for the components $\tilde{\boldsymbol{d}}_{n \omega}$ (with arbitrary $n$ ) in (119). The clearest physical interpretation of $\tilde{\boldsymbol{d}}_{n \omega}$ is that $\boldsymbol{e}^{\prime *} \cdot \tilde{\boldsymbol{d}}_{n \omega}$ is the amplitude whereby $n$ laser photons are absorbed by the atom, which then emits (spontaneously) a high-order harmonic photon with polarization vector $\boldsymbol{e}^{\prime}$ [168]. In contrast, the expectation value of $\boldsymbol{r}$ diverges for the QQES and, in a strong laser field, is not connected with a physical observable. 

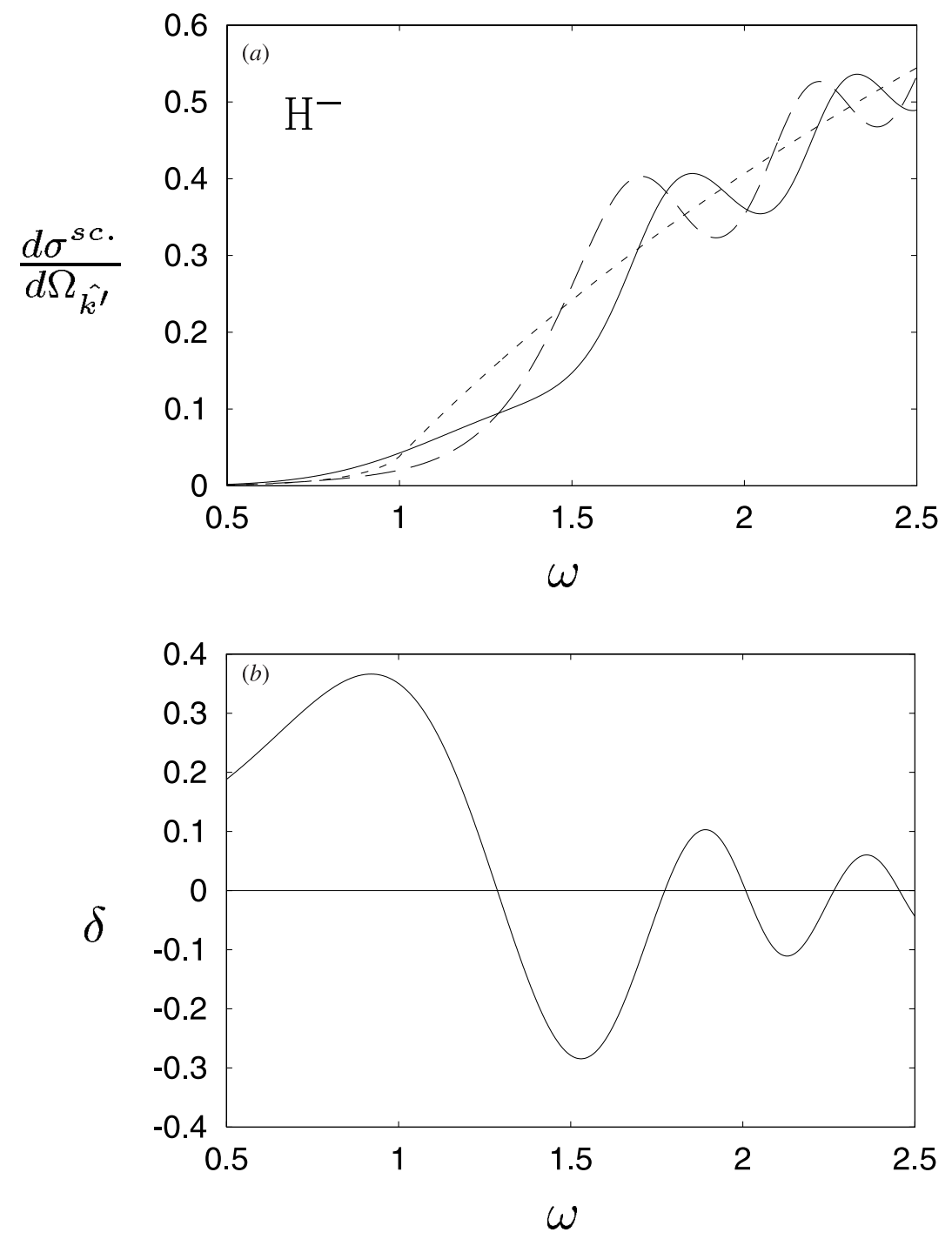

Figure 3. The same as figure 2, but for $\mathcal{F}=0.15$.

\section{Analysis of the dynamic polarizability $\alpha(\mathcal{F} ; \omega)$}

To analyse the $\mathcal{F}$ and $\omega$ dependence of $\alpha(\mathcal{F} ; \omega)$, it is convenient to write the matrix elements (58) and (59) in terms of $g_{\mathcal{F}}(E)=-\pi \mathcal{F}^{-1 / 3} J(\xi)$ and its derivatives,

$$
\begin{aligned}
& g_{\mathcal{F}}^{\prime}(E)=-\pi \mathcal{F}^{-1 / 3} I(\xi)=-\pi \mathcal{F}^{-1 / 3} \operatorname{Ai}(\xi) \operatorname{Ci}(\xi) \\
& g_{\mathcal{F}}^{\prime \prime}(E)=\pi \mathcal{F}^{-1} I^{\prime}(\xi)=\pi \mathcal{F}^{-1}\left(\operatorname{Ai}^{\prime}(\xi) \operatorname{Ci}(\xi)+\operatorname{Ai}(\xi) \mathrm{Ci}^{\prime}(\xi)\right)
\end{aligned}
$$

as follows:

$$
M_{ \pm 1,0}(-E)=\mathrm{e}^{\mp \mathrm{i} \phi_{1}} \Lambda \frac{\gamma \mathcal{F}}{2 \omega}\left(g_{\mathcal{F}}^{\prime}(E)+g_{\mathcal{F}}^{\prime}(E \mp \omega)\right)
$$


$M_{0,0}(-E)=-\frac{2 \gamma^{2}}{3 \omega^{2}}\left[\Pi(\mathcal{F}, \omega)-\frac{3}{8} \Lambda^{2} \mathcal{F}^{2} \sum_{n=-1}^{1} C_{2}^{n+1} g_{\mathcal{F}}^{\prime \prime}(E+n \omega)\right]$.

Note that in these equations we have replaced $E^{\prime}$ in (58) and (59) by $-E$. Here $C_{2}^{n+1}$ is the binomial coefficient and we have introduced the function

$\Pi(\mathcal{F}, \omega)=\sum_{n=-1}^{1}(-1)^{n} C_{2}^{n+1}\left[(E+n \omega) g_{\mathcal{F}}(E+n \omega)+\frac{1}{4} \mathcal{F}^{2} g_{\mathcal{F}}^{\prime \prime}(E+n \omega)\right]$.

With these definitions and using (126), (127) and (130) $\alpha(\mathcal{F} ; \omega)$ can be presented in the form

$$
\begin{aligned}
\alpha(\mathcal{F} ; \omega)=-\frac{2}{\omega^{2}}-\frac{1}{\omega^{4} g_{\mathcal{F}}^{\prime}(E)}\left\{\frac{4}{3} \Pi(\mathcal{F}, \omega)-\Lambda^{2} \mathcal{F}^{2} g_{\mathcal{F}}^{\prime \prime}(E)\right. \\
\left.\quad-\frac{1}{2} \Lambda^{2} \mathcal{F}^{2} \sum_{k= \pm 1}\left[g_{\mathcal{F}}^{\prime \prime}(E+k \omega)+\frac{\left(g_{\mathcal{F}}^{\prime}(E)+g_{\mathcal{F}}^{\prime}(E+k \omega)\right)^{2}}{1-g_{\mathcal{F}}(E+k \omega)}\right]\right\} .
\end{aligned}
$$

As pointed out above, for weak fields $\mathcal{F}$ the energy $E$ in this equation should be changed to $E_{0}=-1$.

For $\mathcal{F}=0$ the function $g_{\mathcal{F}}$ (together with its derivatives) is $\mathcal{F}$ independent. Thus, by using the relation $\left.g_{\mathcal{F}}(E)\right|_{\mathcal{F}=0}=\sqrt{-E}$, the expression (141) may be transformed immediately to the well known result for the polarizability of a weakly bound particle,

$$
\alpha_{0}(\omega)=-\frac{2}{\omega^{2}}-\frac{16}{3 \omega^{4}}+\frac{8}{3 \omega^{4}}\left[(\omega+1)^{3 / 2}+\mathrm{i}(\omega-1)^{3 / 2}\right]
$$

which is independent of the ellipticity of the laser field [105].

For $\mathcal{F} \neq 0$ the analytical structure of the expression (141) for $\alpha(\mathcal{F} ; \omega)$ is also very simple and contains only regular and irregular Airy functions and their first derivatives. One sees that for an arbitrary geometry, the polarization dependence of $\alpha(\mathcal{F} ; \omega)$ is very weak. Indeed, the parameter $\Lambda^{2}$ changes from $\sin ^{2} \theta$ for the case of circular polarization of $\boldsymbol{F}(t)$ to $\Lambda_{l}^{2}=2 \sin ^{2} \theta \cos ^{2} \phi=2 \cos ^{2} \Theta$ for the case of linear polarization (where $\Theta$ is the angle between $\mathcal{F}$ and $\boldsymbol{F}(t)$ ). Since an arbitrarily oriented static field breaks the spherical symmetry, the difference between the selection rules for dipole transitions for either linearly or circularly polarized light is not so important as for $\mathcal{F}=0$. The result (141) is simplified significantly for orthogonal fields, $\mathcal{F} \cdot \boldsymbol{F}(t)=0$. In this case only the term with $\Pi$ contributes to the result, which is independent of the polarization of the light field. In this geometry our result for the polarizability coincides with the result of [24], which was derived only for the special case of circularly polarized $\boldsymbol{F}(t)$ orthogonal to $\mathcal{F}$. The dependence of $\alpha(\mathcal{F} ; \omega)$ on the angle between $\mathcal{F}$ and $\boldsymbol{F}(t)$ ) (i.e. on the parameter $\Lambda^{2}=\sin ^{2} \theta(1+l \cos 2 \phi)$ ) is very significant. Figures 4 and 5 demonstrate the occurrence of oscillatory structure in the frequency dependence of both real and imaginary parts of the polarizability with increasing values of $\Lambda$.

Obviously, in the presence of a static field there is no threshold for the photoeffect, and $\alpha(\mathcal{F} ; \omega)$ has a non-zero imaginary part at any frequency $\omega$. For frequencies far from the ionization threshold $(\omega<1)$, the corresponding level width, $\Gamma=\operatorname{Im} \alpha(\mathcal{F}, \omega) F^{2}$, gives only small corrections $\propto F^{2}$ to the field ionization width, $\Gamma_{\mathcal{F}}$, determined by the imaginary part of $\Delta E^{\text {stat }}$ in (49). However, for frequencies near and above threshold the width $\Gamma$ can differ from $\Gamma_{\mathcal{F}}$ even for moderate values of $\mathcal{F}$. The physical reason for this is clear: at $F=0$ the unique method of decay for a bound level is tunnelling ionization. For $F \neq 0$ and for below-threshold frequencies, in addition to the 'direct' tunnelling mechanism, we also have tunnelling from the harmonic of the quasienergy state with initial 'energy' $E+\omega \equiv \Delta<0$. 

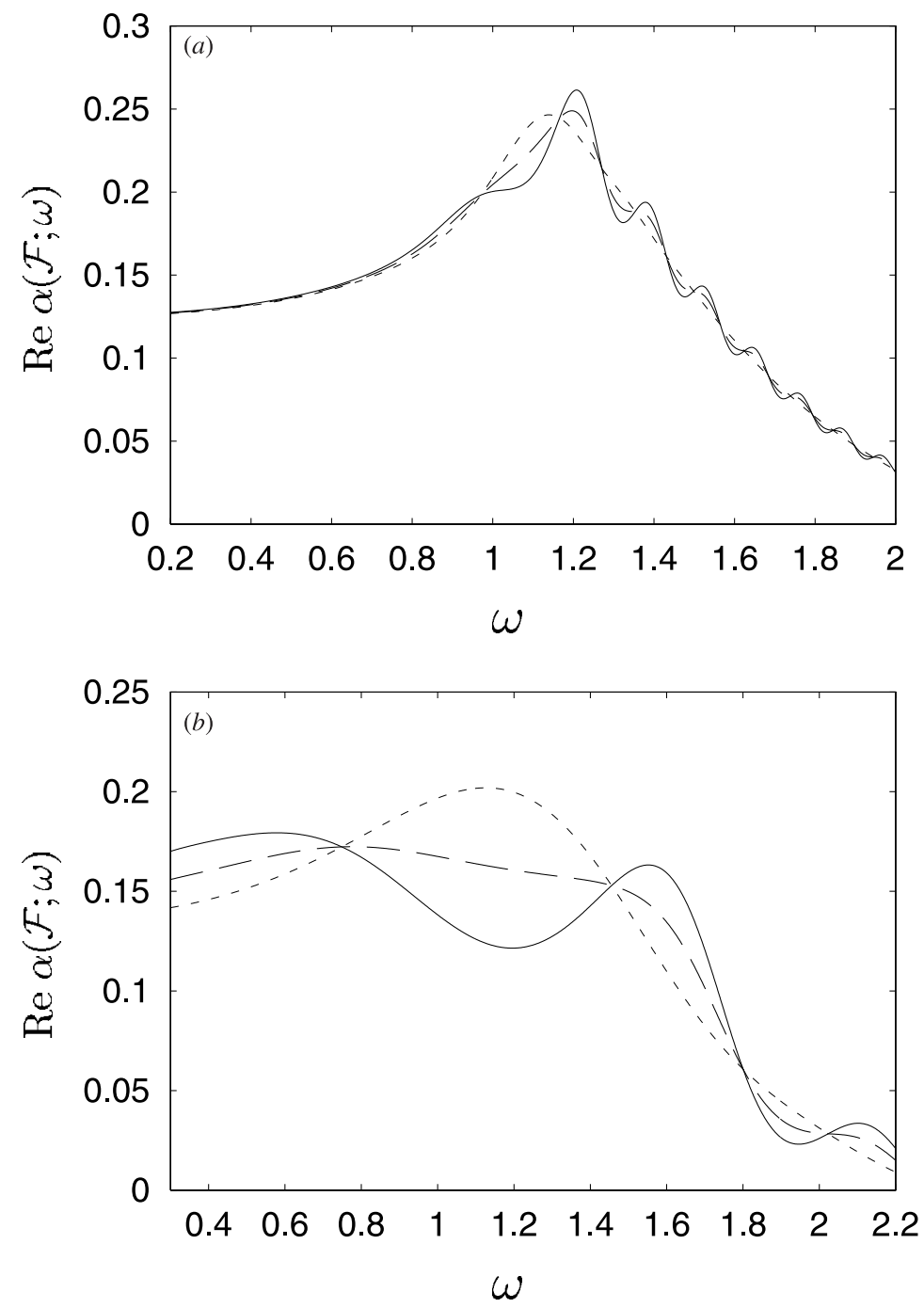

Figure 4. Frequency and field geometry dependence of $\operatorname{Re} \alpha(\mathcal{F} ; \omega)$, (141), for $(a) \mathcal{F}=0.03$ and (b) $\mathcal{F}=0.15$. The parameter $\Lambda$ (see (20)) is $\Lambda=\sqrt{2}$ (full curve), $\Lambda=1$ (broken curve) and $\Lambda=0$ (dotted curve).

Such a mechanism was analysed in [25] and it was shown there that for $|\Delta| \ll|E|$ this channel dominates over the 'direct' one even for $F \ll \mathcal{F}$. Finally, for $\omega \geqslant 1$ we have the ordinary photoeffect modified by the well known interference (oscillation) effects caused by the static field. All of these channels are described by imaginary parts of the $g_{\mathcal{F}}(x)$-functions in (141) with $x=E$ and $E+\omega$. Note that the imaginary part of $g_{\mathcal{F}}(E-\omega)$ is also non-zero and it corresponds to tunnelling with stimulated emission of a photon, but evidently this channel is much more suppressed than the 'direct' one because of a higher barrier. A detailed analysis of stimulated emission is presented in [51]. 

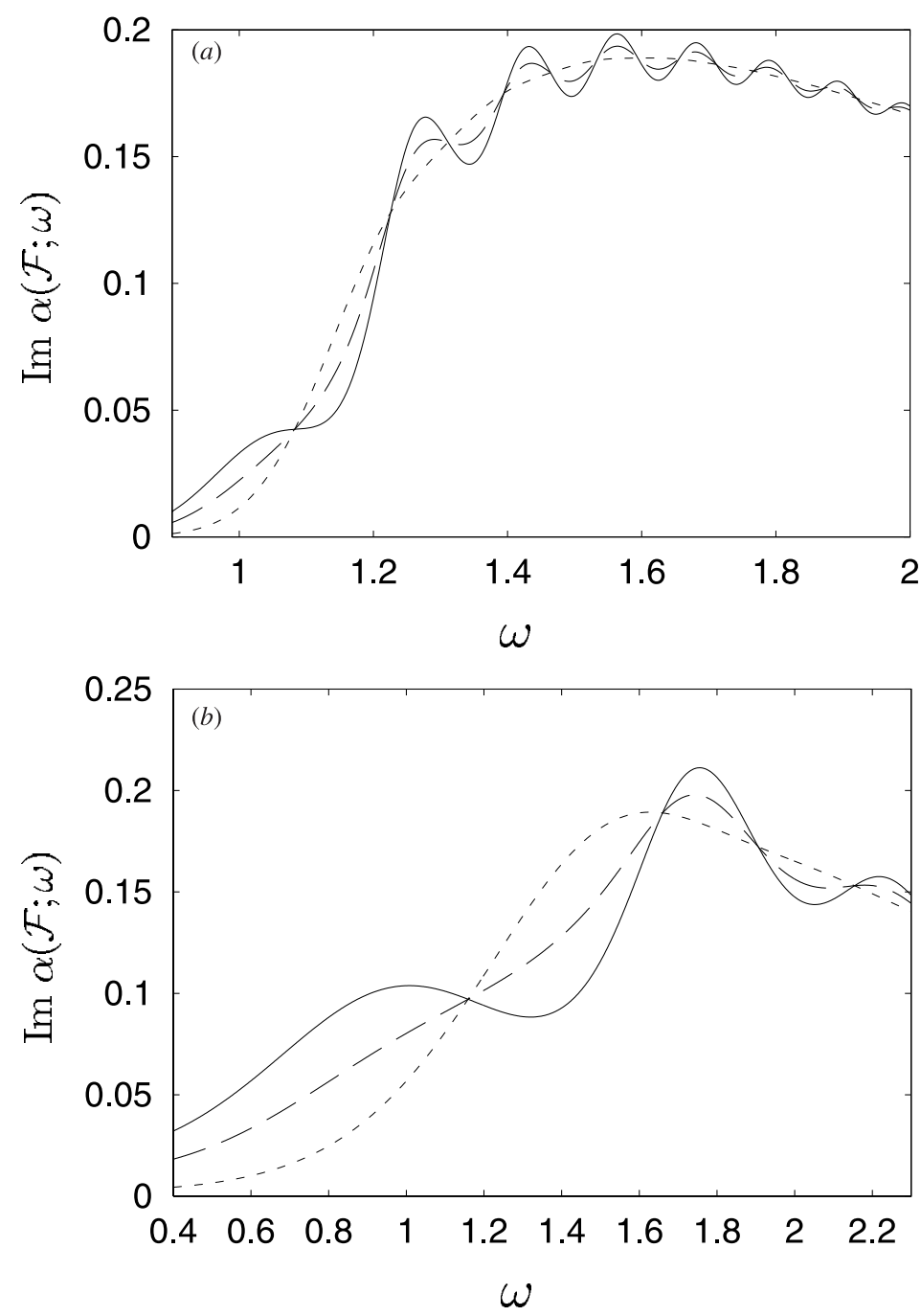

Figure 5. The same as in figures $4(a)$ and $(b)$ for $\operatorname{Im} \alpha(\mathcal{F} ; \omega)$.

\subsection{Below- and above-threshold asymptotics}

Using the asymptotic expansions for $g_{\mathcal{F}}(E)$ and its derivatives for large negative and positive arguments (see appendix C), limiting expressions of the exact result (141) for $\alpha(\mathcal{F} ; \omega)$ may be obtained for frequencies both below and above threshold for weak static electric fields. Neglecting static-field-ionization effects, which are exponentially small for $\mathcal{F}$ not too strong, the energy $E$ in terms of the right-hand side of (141) (including in the arguments of the Airy functions) becomes equal to $-1-\mathcal{F}^{2} / 16$ on account of the quadratic Stark shift (see (48)). Furthermore, we may neglect (small) exponential terms in the asymptotic expansions of the Airy functions and their derivatives involving the arguments $\xi=-E \mathcal{F}^{-2 / 3}$ and $\xi_{+1}=-(E-\omega) \mathcal{F}^{-2 / 3}$. 
For $\omega<1$ and $\xi_{-1} \equiv(E-\omega) \mathcal{F}^{-2 / 3} \gg 1$ we find in this way that

$$
\begin{aligned}
\operatorname{Re} \alpha(\mathcal{F} ; \omega)= & \alpha_{0}(\omega)-\frac{\mathcal{F}^{2}}{12 \omega^{3}}\left[\frac{4 \omega^{2}+9 \omega+6}{(1+\omega)^{3 / 2}}-\frac{4 \omega^{2}-9 \omega+6}{(1-\omega)^{3 / 2}}\right] \\
& -\frac{\mathcal{F}^{2} \Lambda^{2}}{4 \omega^{4}}\left[\frac{2 \omega^{2}}{1-\omega^{2}}-\frac{\omega^{2}+6 \omega+4}{\omega(1+\omega)^{3 / 2}}+\frac{\omega^{2}-6 \omega+4}{\omega(1-\omega)^{3 / 2}}\right]
\end{aligned}
$$

$\operatorname{Im} \alpha(\mathcal{F} ; \omega)=\left[\frac{\Lambda^{2} \mathcal{F}}{2 \omega^{4}}+\frac{\mathcal{F}^{2}}{4 \omega^{4}(1-\omega)^{3 / 2}}\right] \mathrm{e}^{-\frac{4}{3} \xi_{-1}^{3 / 2}}$

where $\Lambda$ is defined in (20). Thus, in the below-threshold region, taking account of the static electric field leads to corrections for the real part of the polarizability that are quadratic in $\mathcal{F}$. Note that these corrections depend on both the polarization and the geometry of the fields. The imaginary part of $\alpha(\mathcal{F} ; \omega)$ describes 'tunnelling from the virtual state with an energy $\left(E_{0}+\omega\right)^{\prime}$, i.e. the electron escapes by tunnelling in the static electric field after the absorption of a laser photon. The difference in equation (144) for $\Lambda=0$ and $\Lambda \neq 0$ is associated with the fact that for orthogonal fields $(\Lambda=0)$ the angular momentum of the electron after the photon is absorbed has the projection $m= \pm 1$ in the direction of $\mathcal{F}$, while the tunnelling width is proportional to $\mathcal{F}^{1+|m|}$ (cf, e.g., [176]). Therefore, for below-threshold frequencies the ionization width is maximum for coplanar fields $(\Lambda \neq 0)$ owing to the linear dependence on $\mathcal{F}$ of the coefficient multiplying the exponential in (144). In other words, the tunnelling barrier is reduced for coplanar fields.

For above-threshold frequencies $(\omega>1)$ and $(\omega-1) \mathcal{F}^{-2 / 3} \gg 1$, the Airy functions have oscillatory asymptotic behaviours which lead to oscillatory behaviour in both the real part and the imaginary part of the polarizability,

$\operatorname{Re} \alpha(\mathcal{F} ; \omega)=\operatorname{Re} \alpha_{0}(\omega)-\frac{\mathcal{F} \Lambda^{2}}{\omega^{4}} \sin \chi-\frac{\mathcal{F}^{2}}{12 \omega^{3}}\left[\frac{6 \cos \chi}{\omega(\omega-1)^{3 / 2}}+\frac{4 \omega^{2}+9 \omega+6}{(1+\omega)^{3 / 2}}\right]$

where

$\operatorname{Re} \alpha_{0}(\omega)=-\frac{2}{\omega^{2}}\left[1+\frac{4}{3 \omega^{2}}\left\{2-(1+\omega)^{3 / 2}\right\}\right]$

$\operatorname{Im} \alpha(\mathcal{F} ; \omega)=\operatorname{Im} \alpha_{0}(\omega)+\frac{\mathcal{F} \Lambda^{2}}{\omega^{4}} \cos \chi-\frac{\mathcal{F}^{2}}{12 \omega^{3}}\left[\frac{6 \sin \chi}{\omega(\omega-1)^{3 / 2}}+\frac{4 \omega^{2}-9 \omega+6}{(\omega-1)^{3 / 2}}\right]$

where

$$
\operatorname{Im} \alpha_{0}(\omega)=\frac{8(\omega-1)^{3 / 2}}{3 \omega^{4}} \quad \chi \equiv \frac{4}{3} \frac{(\omega-1)^{3 / 2}}{\mathcal{F}} .
$$

Note, that the oscillatory structure of the photodetachment cross section is known from previous studies (see [24, 31, 34]), in which it is explained by static-field-induced quantum interference effects. Namely, detached electrons can escape by two different paths (a direct path and one involving reflection by a static field), whose amplitudes interfere with each other when calculating the combined detachment amplitude.

Figures 6 and 7 present a comparison of the exact result for $\alpha(\mathcal{F} ; \omega)$, calculated using (141), with results obtained using the above approximations. Evidently, the approximate equations (143)-(146) give results which are inapplicable near the zero-field threshold, when the parameter $|\omega-1| \mathcal{F}^{-2 / 3}$ is small. For non-zero $\mathcal{F}$ the typical (for a short-range potential) square-root threshold peculiarity (i.e. the branch point) of $\alpha_{0}(\omega)$ at $\omega=1$ vanishes and $\alpha(\mathcal{F} ; \omega)$ is analytic in $\omega$ for finite $\mathcal{F}$. Nevertheless, for not too strong $\mathcal{F}$, the behaviour of $\alpha$ has a specific 

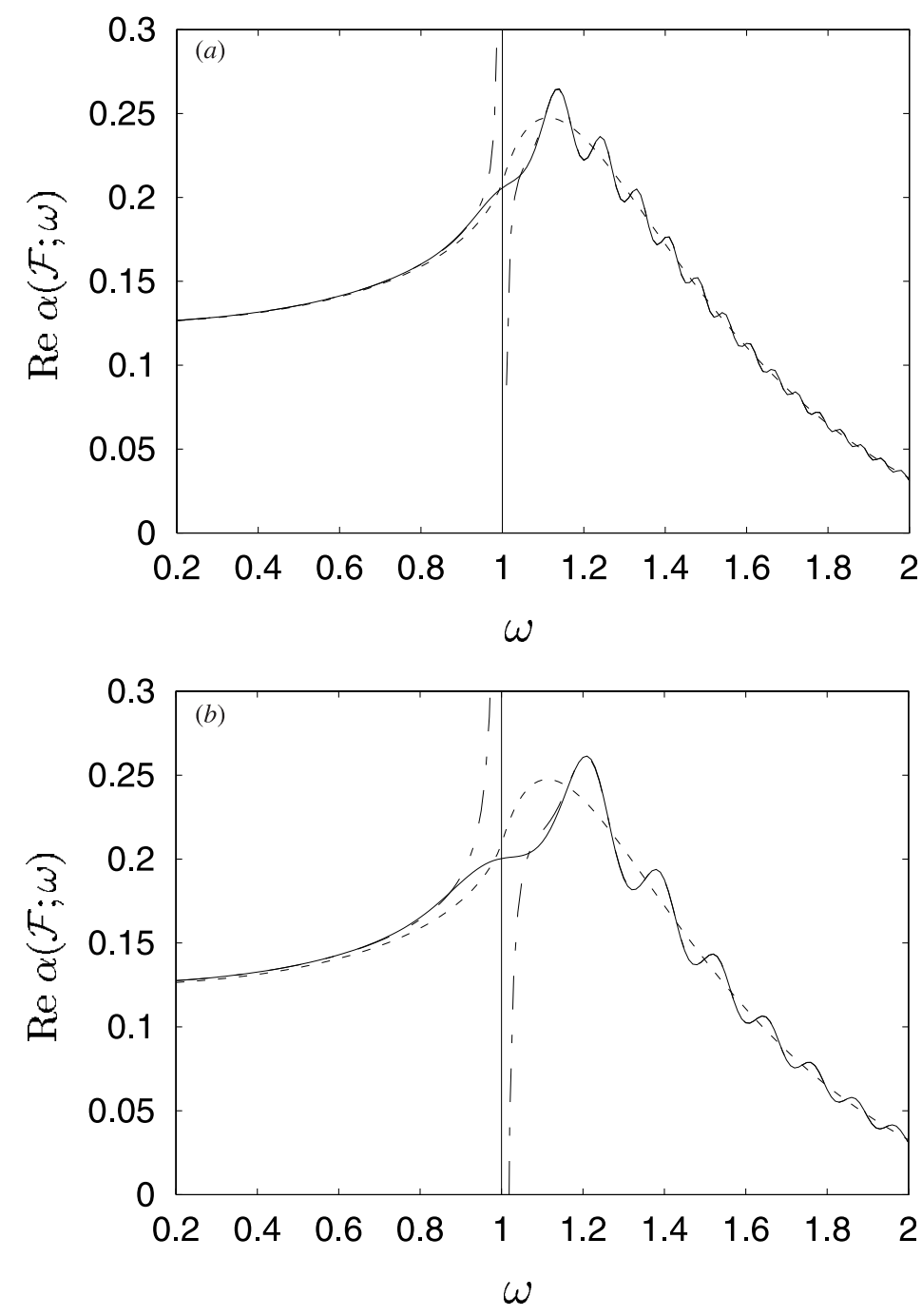

Figure 6. Frequency and $\mathcal{F}$ dependence of $\operatorname{Re} \alpha(\mathcal{F} ; \omega)$ for $\Lambda=\sqrt{2}$ and $(a) \mathcal{F}=0.015 ;(b) 0.03$; (c) 0.06 ; $(d) 0.15$. Full curve, the exact result (141); dotted curve, the result (142) for $\mathcal{F}=0$; chain curve, analytical approximations (143) and (145).

form near $\omega=1$ which exhibits some 'memory' of the $\mathcal{F}=0$ threshold behaviour. For example, this point separates the intervals of exponential and oscillatory frequency dependence of $\operatorname{Im} \alpha(\mathcal{F} ; \omega)$.

\subsection{Behaviour of $\alpha(\mathcal{F} ; \omega)$ near and at threshold}

The threshold (and the near-threshold) behaviour of $\alpha(\mathcal{F} ; \omega)$ is analysed easily using the expansions of Airy functions for small arguments (see appendix C). Taking into account the 

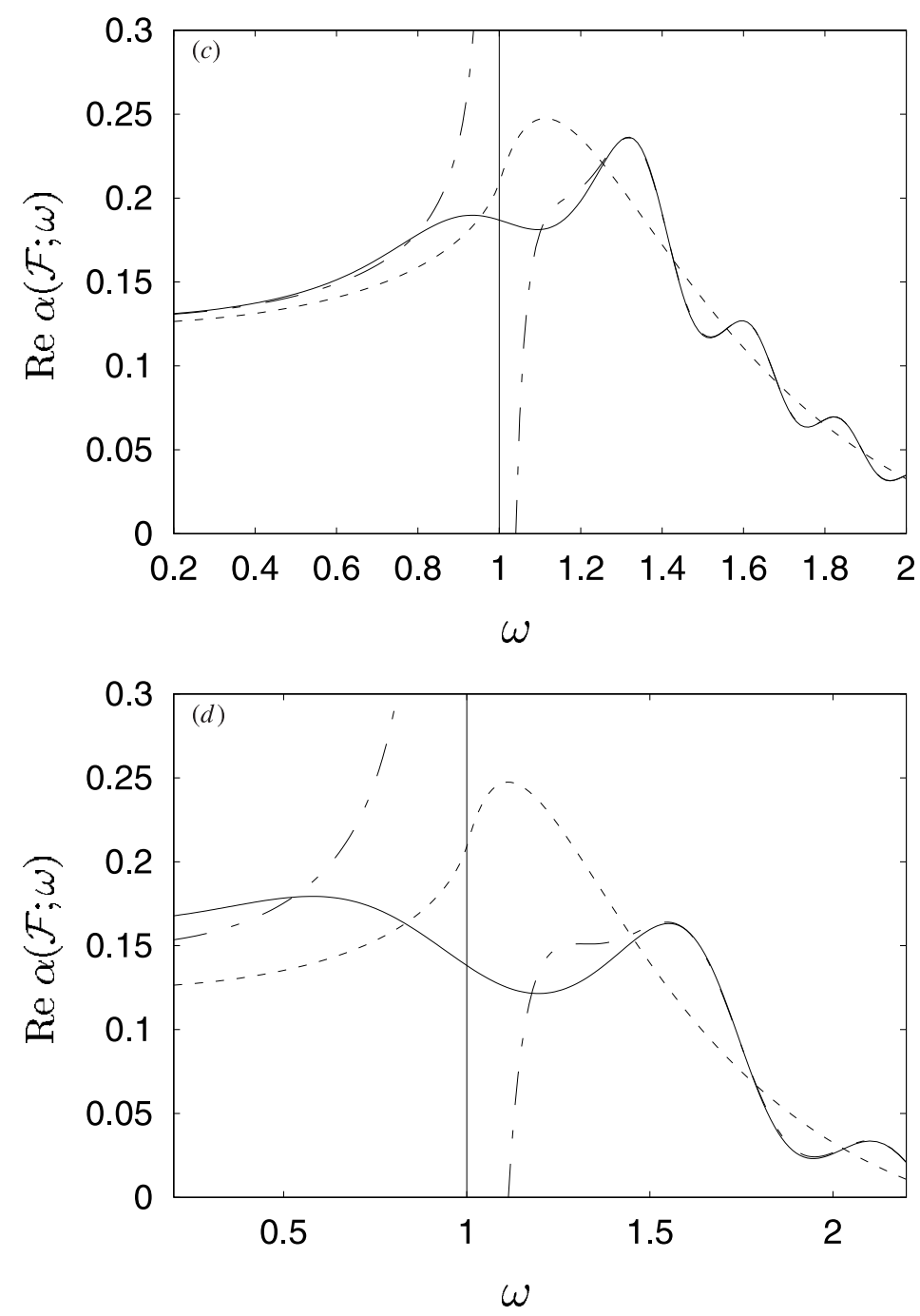

Figure 6. Continued.

Stark shift of $E_{0}$, the result for $\alpha(\mathcal{F} ; \omega=1) \equiv \alpha^{\text {th }}(\mathcal{F})$ is $\operatorname{Re} \alpha^{\text {th }}(\mathcal{F})=\frac{2}{3}(8 \sqrt{2}-11)-\frac{\Lambda^{2}}{6 c_{0}^{2}} \mathcal{F}^{4 / 3}-\frac{2 \Lambda^{2}}{3 c_{0}} \mathcal{F}^{5 / 3}-\frac{1}{48}\left[19 \sqrt{2}-\frac{1}{3} \Lambda^{2}(224+189 \sqrt{2})\right] \mathcal{F}^{2}$

$\operatorname{Im} \alpha^{\text {th }}(\mathcal{F})=\frac{2}{3 \sqrt{3}}\left(1+\frac{3}{2} \Lambda^{2}\right) \mathcal{F}-\frac{\Lambda^{2}}{2 \sqrt{3} c_{0}^{2}} \mathcal{F}^{4 / 3}-\frac{2 \Lambda^{2}}{3 \sqrt{3} c_{0}} \mathcal{F}^{5 / 3}-\frac{c_{0}}{8 \sqrt{3}}\left[1-\frac{41}{18} \Lambda^{2}\right] \mathcal{F}^{7 / 3}$ 

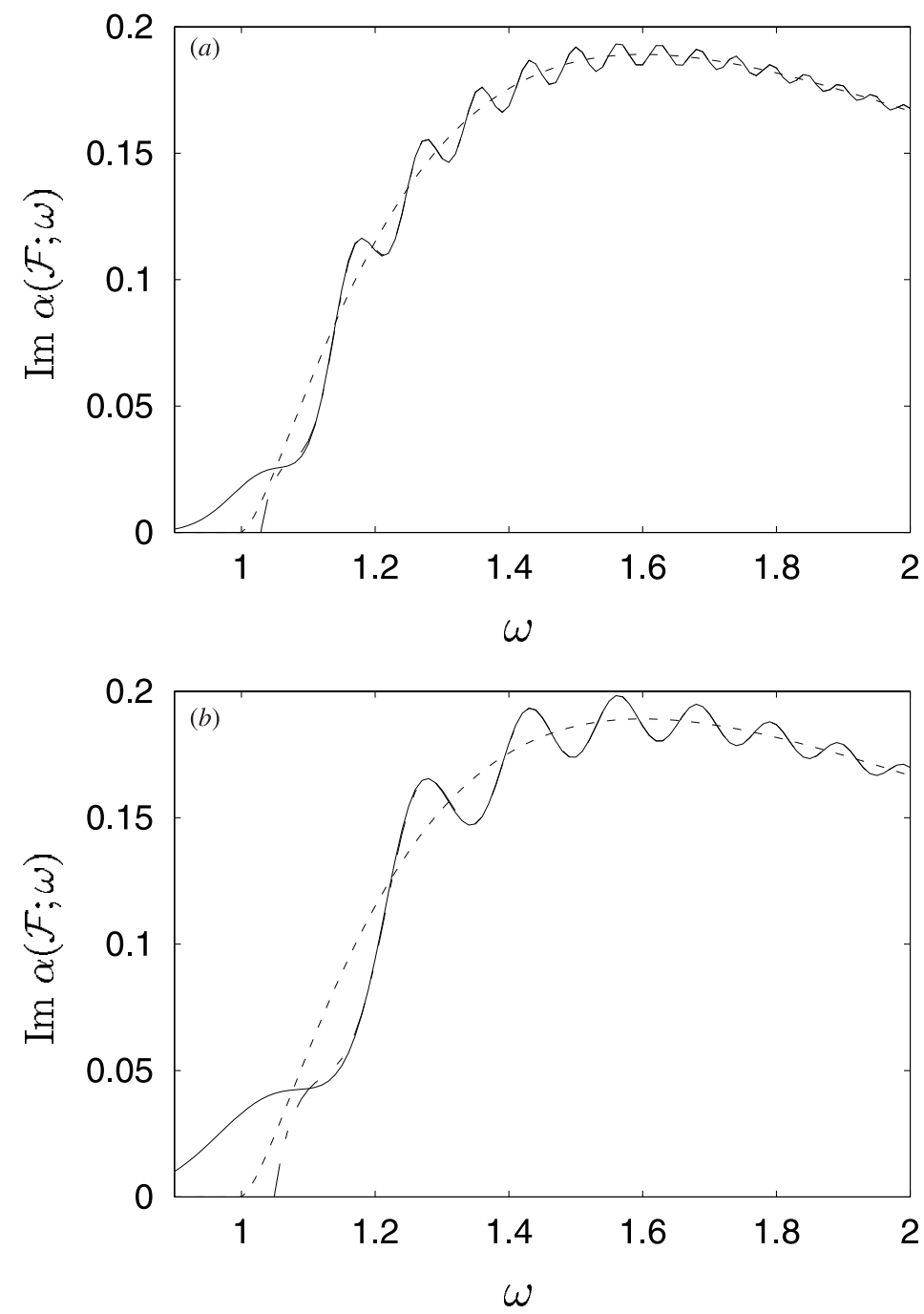

Figure 7. The same as figures $6(a),(b),(c)$ and $(d)$ for $\operatorname{Im} \alpha(\mathcal{F} ; \omega)$. Chain curve, analytical approximation (146).

where $c_{0}=\left(3^{5 / 6} / 2 \pi\right)\left[\Gamma\left(\frac{2}{3}\right)\right]^{2} \approx 0.729$. The generalization of results (147) and (148) for near-threshold frequencies $\left(|\omega-1| \ll \mathcal{F}^{2 / 3}\right)$ is

$$
\alpha(\mathcal{F} ; \omega)=\alpha^{\text {th }}(\mathcal{F})+\left.\frac{\partial \alpha(\mathcal{F} ; \omega)}{\partial \omega}\right|_{\omega=1}(\omega-1)
$$

where

$$
\begin{aligned}
\left.\operatorname{Re} \frac{\partial \alpha(\mathcal{F} ; \omega)}{\partial \omega}\right|_{\omega=1} & =\frac{4}{3}(13 \sqrt{2}-22)+c_{0}\left(2+\Lambda^{2}\right) \mathcal{F}^{1 / 3}-\frac{\Lambda^{2}}{3 c_{0}} \mathcal{F}^{2 / 3} \\
& +\frac{1}{9} \Lambda^{2}\left(\frac{25}{2} c_{0}-\frac{16}{c_{0}^{2}}\right) \mathcal{F}^{4 / 3}-\frac{8 \Lambda^{2}}{3 c_{0}} \mathcal{F}^{5 / 3} \\
& -\frac{1}{12}\left[19 \sqrt{2}-\frac{1}{3} \Lambda^{2}(224+189 \sqrt{2})\right] \mathcal{F}^{2}
\end{aligned}
$$



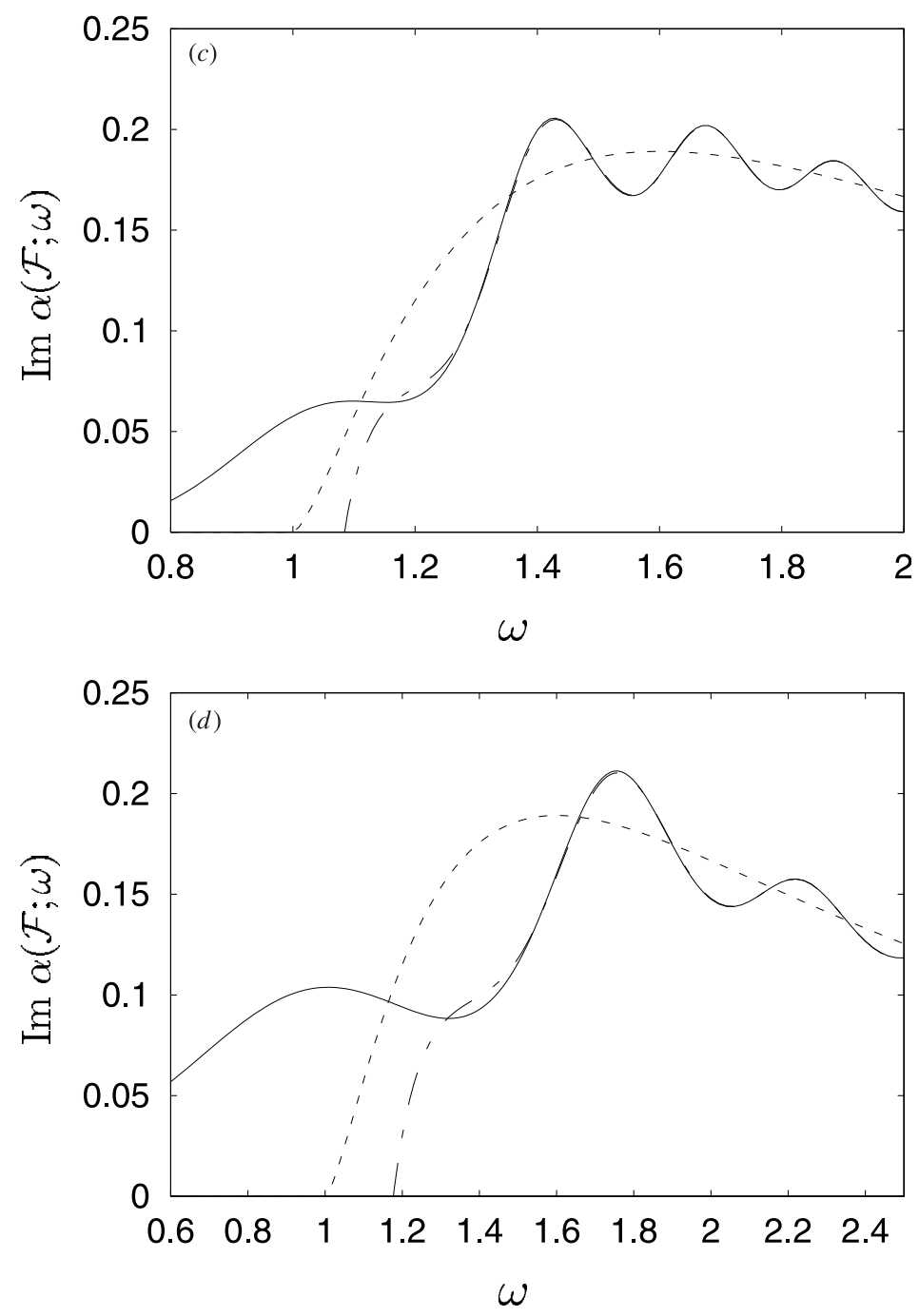

Figure 7. Continued.

$$
\begin{aligned}
\left.\operatorname{Im} \frac{\partial \alpha(\mathcal{F} ; \omega)}{\partial \omega}\right|_{\omega=1} & =\frac{\mathcal{F}^{1 / 3}}{\sqrt{3}}\left\{c_{0}\left(2+\Lambda^{2}\right)-\frac{\Lambda^{2}}{c_{0}} \mathcal{F}^{1 / 3}-\frac{1}{3}\left[8+\Lambda^{2}\left(17-\frac{1}{c_{0}^{3}}\right)\right] \mathcal{F}^{2 / 3}\right. \\
& -\frac{1}{6} c_{0} \Lambda^{2}\left(5-\frac{17}{c_{0}^{3}}\right) \mathcal{F}+\frac{1}{8 c_{0}}\left[1+\Lambda^{2}\left(\frac{5}{2} c_{0}^{3}-\frac{161}{6}\right)\right] \mathcal{F}^{4 / 3} \\
& \left.+\frac{1}{2} c_{0}\left(1-\frac{41}{48} \Lambda^{2}\right) \mathcal{F}^{2}\right\} .
\end{aligned}
$$

Figures 8 and 9 present the $\mathcal{F}$ dependence of $\alpha^{\text {th }}(\mathcal{F})$. Note that as $\mathcal{F}$ increases, the first maximum in the frequency dependence of $\operatorname{Im} \alpha(\mathcal{F} ; \omega)$ shifts to the below-threshold domain (see figure 7) and the derivative $\left.(\partial / \partial \omega) \operatorname{Im} \alpha(\mathcal{F} ; \omega)\right|_{\omega=1}$ changes its $\operatorname{sign}\left(\right.$ at $\mathcal{F}=\mathcal{F}^{(0)}=0.2678$ ). This fact explains the slow decrease of $\operatorname{Im} \alpha^{\text {th }}(\mathcal{F})$ in figure 9 as $\mathcal{F}$ becomes larger than $\mathcal{F}^{(0)}$. Note the considerable difference of our results in figure 9 from the approximate results $[41,51]$. 


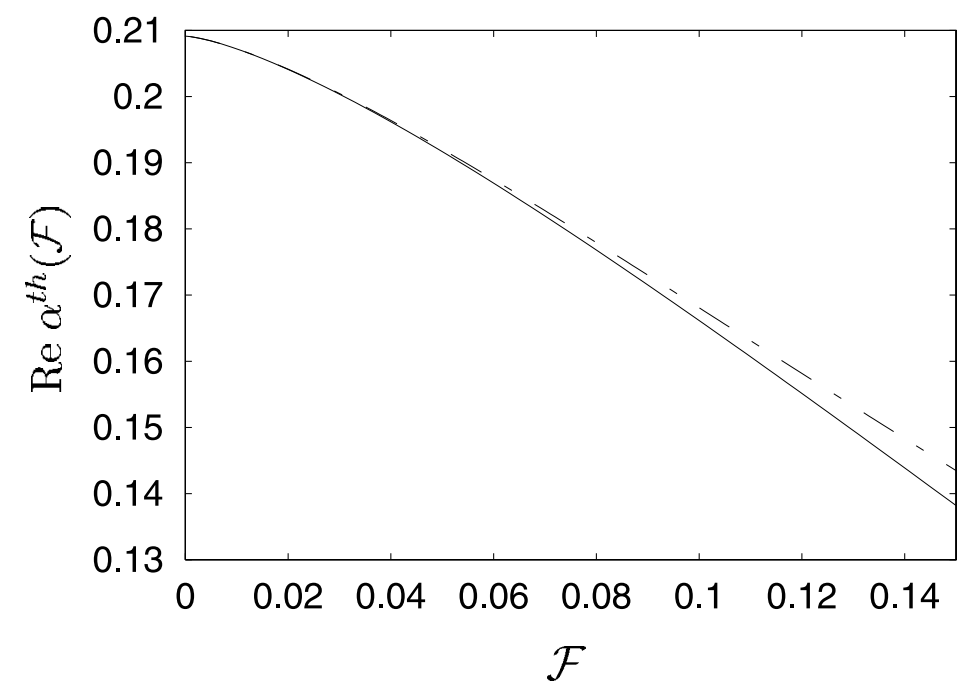

Figure 8. Static field dependence of the threshold value $\operatorname{Re} \alpha^{\text {th }}(\mathcal{F})=\operatorname{Re} \alpha(\mathcal{F} ; \omega=1)$. Full curve, the exact result (141); chain curve, the asymptotic result (147).

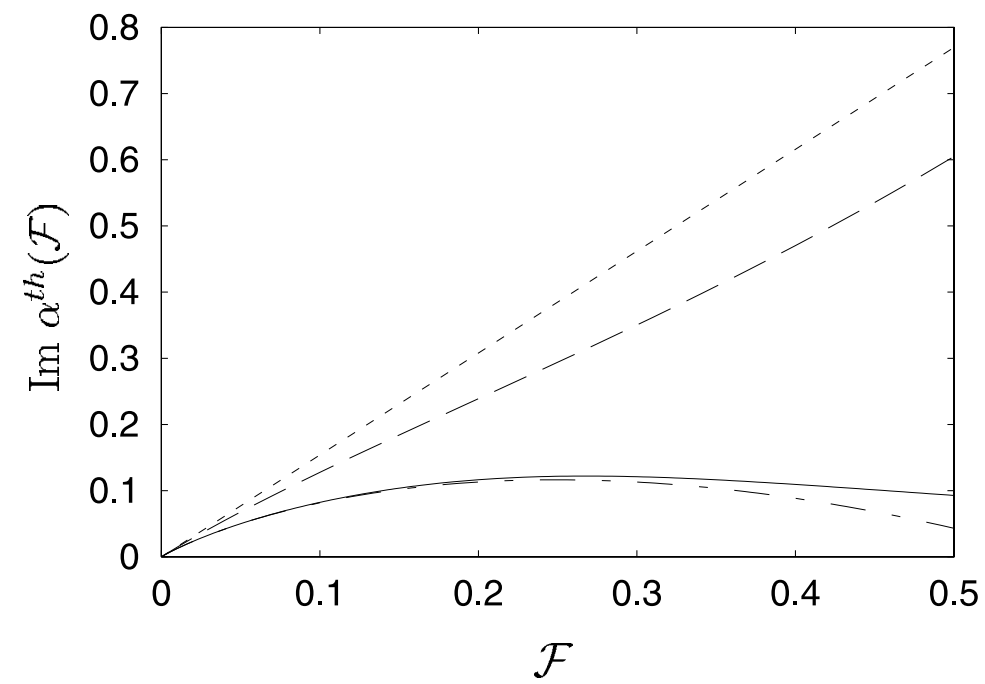

Figure 9. The same as figure 8 for $\operatorname{Im} \alpha^{\text {th }}(\mathcal{F})=\operatorname{Im} \alpha(\mathcal{F} ; \omega=1)$. Chain curve, the asymptotic result (148); dotted curve, the result of Du and Delos [41]; broken curve, the result of Gao and Starace [51].

This difference is caused by two circumstances which are significant for the threshold case. Firstly, there is a 'rescattering' effect, which is neglected in both cited works, which consider the photodetached electron as a free electron in a static electric field. Secondly, there is the 'shift' of the threshold frequency, $\omega=\left|E_{0}\right|=1$, in a static electric field, which is taken into account in the exact result (141) by using $E$ instead of $E_{0}$.

In figure 10 we present the $\mathcal{F}$ dependence of the 'dynamical' threshold frequency, $\omega^{\text {th }}=|\operatorname{Re} E|$, calculated from the exact equation (47) for (complex) $E$. 


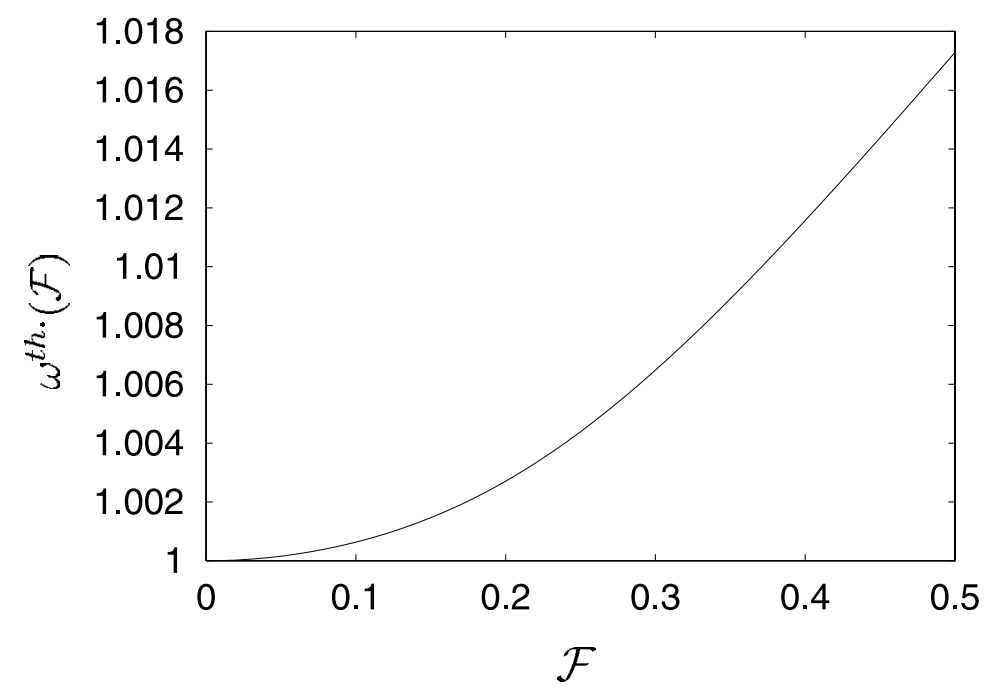

Figure 10. $\mathcal{F}$ dependence of the static-electric-field-modified threshold frequency, $\omega^{\text {th }}=|\operatorname{Re} E|$, calculated from (47).

\section{The photodetachment cross section and connections with previous studies}

\subsection{General considerations}

For zero static field the imaginary part of the dynamic polarizability is connected with the photodetachment cross section $\sigma_{0}(\omega)$ in accordance with the optical theorem. For $\mathcal{F}=0$, (142) gives for $\omega>1$ :

$$
4 \pi \alpha \omega \operatorname{Im} \alpha_{0}(\omega)=\sigma_{0}(\omega)=\frac{32 \pi \alpha}{3 \omega^{3}}(\omega-1)^{3 / 2}
$$

where $\alpha$ is the fine structure constant and $\sigma_{0}(\omega)$ is the well known result for the photodetachment cross section of a weakly bound system. This result was found by Bethe and Peierls in 1935 [177] in their analysis of the photodisintegration of the deuteron. Later this simple formula was used widely in the study of negative ion photodetachment (see, e.g., [178]). In what follows, we discuss the connection of our approach with previous studies of static-electricfield-induced effects on negative ion photodetachment. This problem is interesting since we have an exemplary case of an exact solution for a problem involving three different interactions, two of which (i.e. the interactions of the electron with an atomic binding potential and with a static electric field) are accounted for exactly and the third of which (the electron's interaction with the laser field) is treated using a PT approach. Thus it is possible to analyse the accuracy of different approximate methods for this problem, thereby shedding light on their applicability to other similar problems.

In contrast to the case of zero static field, the imaginary part of the $\mathcal{F}$-dependent polarizability $\alpha(\mathcal{F} ; \omega)$ of an atom (or ion) involves the total level width $\Gamma \sim F^{2}$ caused by photoionization, stimulated emission and corrections of order $F^{2}$ to static-field ionization. Thus, in general, it is impossible to formulate an accurate prescription (e.g. similar to the 

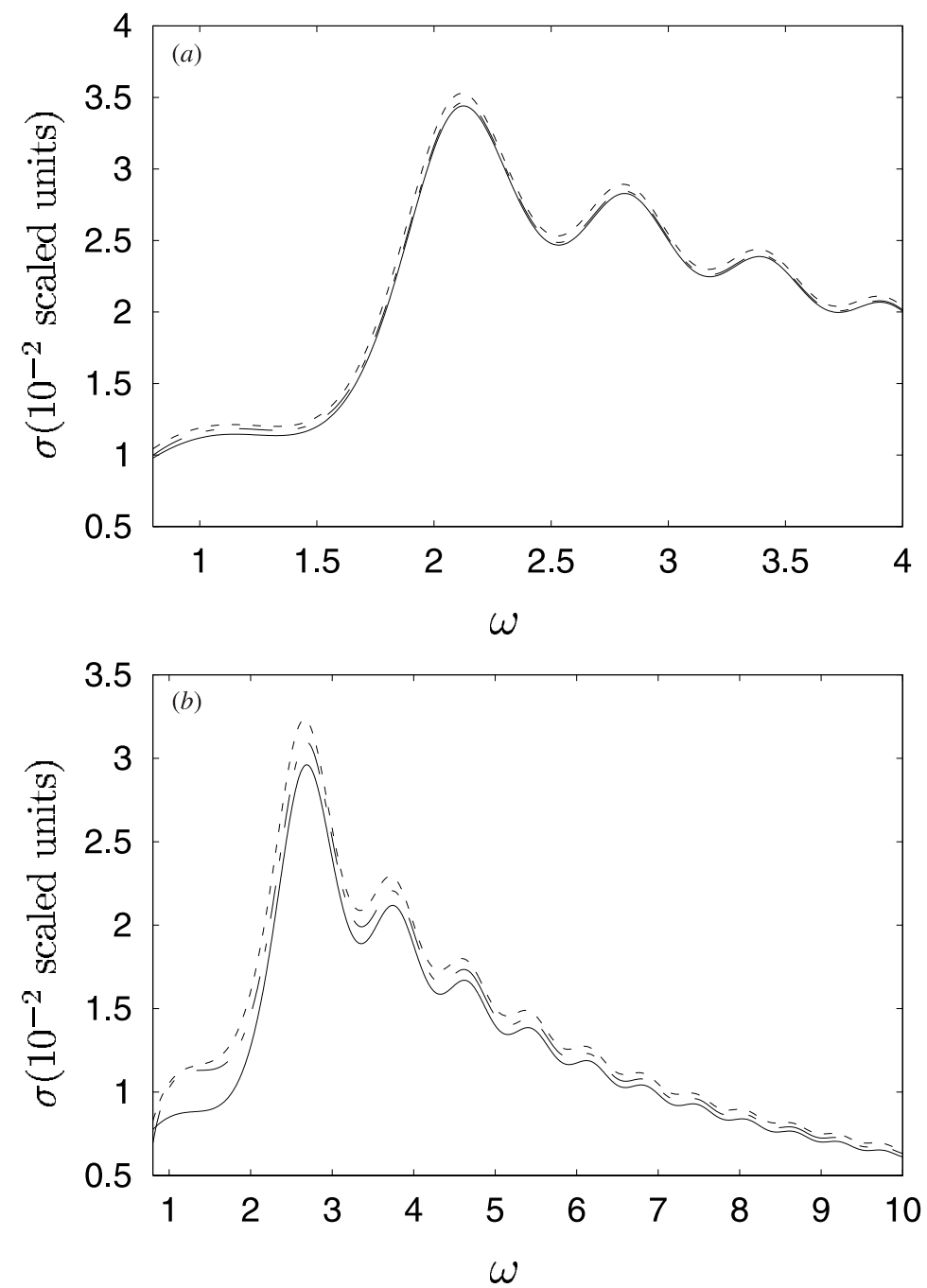

Figure 11. The accuracy of various definitions for the photodetachment cross section for $(a)$ $\mathcal{F}=0.2678$ and $(b) \mathcal{F}=0.5, \Lambda=\sqrt{2}$. Full curve, the definition in (151) with exact $\alpha(\mathcal{F} ; \omega)$ (equation (141)); chain curve, the result in (152) with exact $g_{\mathcal{F}}^{\prime}(E)$; dotted curve, the result in (155).

optical theorem) for the extraction of only the photoionization (or photodetachment) cross section, $\sigma(\mathcal{F}, \omega)$, from $\operatorname{Im} \alpha(\mathcal{F} ; \omega)$. To formally parametrize the total laser-field-induced decay probability in terms of the (total) cross section, $\sigma^{\text {(tot) }}(\mathcal{F}, \omega)$, one may use a relation similar to the optical theorem for the case of $\mathcal{F}=0$,

$$
\sigma^{(\mathrm{tot})}(\mathcal{F}, \omega)=4 \pi \alpha \omega \operatorname{Im} \alpha(\mathcal{F} ; \omega)
$$

where the exact $\alpha(\mathcal{F} ; \omega)$ is given by (141). Obviously, the imaginary part in this equation may be extracted explicitly in terms of Airy functions, but the final analytic result is too cumbersome and is not presented here. Its numerical evaluation, however, is presented in 
figure 11. To connect our result with previous studies of photodetachment, we consider only the 'photoionization channel', neglecting the tunnelling correction and the stimulated emission process. The photodetachment cross section in this approximation may be defined again by the expression (151), where only the photoionization channel is taken into account. Evidently, this result corresponds to the calculation of the imaginary part of the polarizability $\alpha(\mathcal{F} ; \omega)$, taking account only of the photodetachment channel, i.e. neglecting the imaginary part of the $g_{\mathcal{F}}$-function and its derivative $g_{\mathcal{F}}^{\prime \prime}$ with arguments $E$ and $E-\omega$ on the right-hand sides of (140) and (141). In this case the definition (151) yields

$$
\begin{gathered}
\sigma(\mathcal{F}, \omega)=\frac{16 \pi \alpha}{3 \omega^{3}} \operatorname{Im}\left\{\frac { 1 } { g _ { \mathcal { F } } ^ { \prime } ( E ) } \left[(E+\omega) g_{\mathcal{F}}(E+\omega)+\frac{1}{4}\left(1+\frac{3}{2} \Lambda^{2}\right) \mathcal{F}^{2} g_{\mathcal{F}}^{\prime \prime}(E+\omega)\right.\right. \\
\left.\left.+\frac{3}{8} \Lambda^{2} \mathcal{F}^{2} \frac{\left(g_{\mathcal{F}}^{\prime}(E)+g_{\mathcal{F}}^{\prime}(E+\omega)\right)^{2}}{1-g_{\mathcal{F}}(E+\omega)}\right]\right\} .
\end{gathered}
$$

For $\mathcal{F}=0$ this result reduces to (150). Analytical approximations of $\sigma(\mathcal{F}, \omega)$ for far- and nearthreshold frequencies are evident from the results of section 8.1 for $\operatorname{Im} \alpha(\mathcal{F} ; \omega)$. The next level of approximation is to neglect the shift and width of the initial bound state $\left(E \rightarrow E_{0}=-1\right)$ and the imaginary part of the function $g_{\mathcal{F}}^{\prime}(E)$ (i.e. $\left.g_{\mathcal{F}}^{\prime}(E) \rightarrow \operatorname{Re} g_{\mathcal{F}}^{\prime}\left(E_{0}\right)\right)$ in (152). For the special case of orthogonal or parallel static and linearly polarized light fields the result for $\sigma(\mathcal{F}, \omega)$ in this approximation is equivalent to that obtained previously by Slonim and Dalidchik (see equations (13) and (15) in [24]). These authors calculated the standard dipole matrix element for the transition amplitude

$$
T_{f i}=\left\langle\Phi_{f}|F z| \Phi_{E}\right\rangle
$$

with the wavefunctions $\left|\Phi_{E}\right\rangle,\left|\Phi_{f}\right\rangle$ of the $\delta$-model potential in the presence of a static electric field. Moreover, they considered the unperturbed energy $E=E_{0}$ and used only real parts of expressions (77) and (95) for $\Phi_{E_{0}}$ and the normalization factor $f_{0}^{2}$.

To present $\sigma(\mathcal{F}, \omega)$ explicitly in terms of Airy functions, we extract the imaginary part in (152) for $E=E_{0}=-1$ using the following simplification:

$$
2 g_{\mathcal{F}}^{\prime}(E)=-1 \text {. }
$$

This result corresponds to taking account of the first non-vanishing term in the asymptotic expansion (C3) for $\pi I\left(E \mathcal{F}^{-2 / 3}\right) / \mathcal{F}^{1 / 3}$ as $\mathcal{F} \rightarrow 0$. Numerical calculations (cf table 2) show that approximation (154) is accurate up to $\mathcal{F} \approx 1$, and is accurate to within about $10 \%$ at $\mathcal{F}=1$. The approximation (154) is equivalent to neglecting the $\mathcal{F}$ dependence of the normalization factor (95) for the wavefunction $\Phi_{E}(\boldsymbol{r})$. The resulting expression for $\sigma(\mathcal{F} ; \omega)$ can be written in the following form, which is convenient for our discussions:

$$
\sigma(\mathcal{F} ; \omega)=\sigma^{(0)}-\frac{4 \pi^{2} \alpha \mathcal{F}^{4 / 3} \Lambda^{2}}{\omega^{3}\left|1-g_{\mathcal{F}}\left(E_{0}+\omega\right)\right|^{2}}\left[\Phi^{(r)}\left(\xi_{-1}, \mathcal{F}\right)+\Phi^{(\mathrm{i})}\left(\xi_{-1}, \mathcal{F}\right)\right]
$$

where

$$
\begin{aligned}
& \sigma^{(0)}=\frac{32 \pi^{2} \alpha \mathcal{F}}{3 \omega^{3}}\left[\xi_{-1}^{2} \operatorname{Ai}^{2}\left(\xi_{-1}\right)-\xi_{-1} \operatorname{Ai}^{\prime 2}\left(\xi_{-1}\right)-\left(\frac{1}{2}+\frac{3}{4} \Lambda^{2}\right) \operatorname{Ai}\left(\xi_{-1}\right) \operatorname{Ai}^{\prime}\left(\xi_{-1}\right)\right] \\
& \Phi^{(r)}=\pi \operatorname{Ai}^{2}\left(\xi_{-1}\right)\left\{2 \operatorname{Ai}\left(\xi_{-1}\right) \operatorname{Bi}\left(\xi_{-1}\right)+\pi \mathcal{F}^{1 / 3}\left[\operatorname{Ai}^{\prime 2}\left(\xi_{-1}\right)\left(\operatorname{Ai}^{2}\left(\xi_{-1}\right)-\operatorname{Bi}^{2}\left(\xi_{-1}\right)\right)\right.\right. \\
& \left.\left.\quad-\xi_{-1} \operatorname{Ai}^{2}\left(\xi_{-1}\right)\left(\operatorname{Ai}^{2}\left(\xi_{-1}\right)+\operatorname{Bi}^{2}\left(\xi_{-1}\right)\right)+2 \operatorname{Ai}\left(\xi_{-1}\right) \operatorname{Ai}^{\prime}\left(\xi_{-1}\right) \operatorname{Bi}\left(\xi_{-1}\right) \operatorname{Bi}^{\prime}\left(\xi_{-1}\right)\right]\right\}
\end{aligned}
$$


Table 2. The normalization factor $L(\mathcal{F})(\operatorname{cf}(96))$ in the limit $\mathcal{F} \rightarrow 0$, where $E$ is calculated from (47). Results confirm the simplification $2 g_{\mathcal{F}}^{\prime}(E)=-1$ (cf (154)).

\begin{tabular}{ll}
\hline $\begin{array}{l}\text { Static field }^{\mathrm{a}} \\
\mathcal{F}\end{array}$ & $\begin{array}{l}\text { Normalization factor }^{\mathrm{b}} \\
L(\mathcal{F})=-2 g_{\mathcal{F}}^{\prime}(E)\end{array}$ \\
\hline 0.001 & $1.0000+\mathrm{i} 4.36(-580)$ \\
0.005 & $1.0000+\mathrm{i} 7.69(-117)$ \\
0.01 & $1.0000+\mathrm{i} 6.17(-59)$ \\
0.05 & $1.0003+\mathrm{i} 1.27(-12)$ \\
0.1 & $1.0013+\mathrm{i} 7.65(-7)$ \\
0.3 & $1.0142+\mathrm{i} 4.93(-3)$ \\
0.5 & $1.0266+\mathrm{i} 2.636(-2)$ \\
0.7 & $1.0281+\mathrm{i} 5.268(-2)$ \\
1.0 & $1.0177+\mathrm{i} 8.744(-2)$ \\
1.5 & $1.0000+\mathrm{i} 1.286(-1)$ \\
2.0 & $0.9588+\mathrm{i} 1.554(-1)$ \\
\hline
\end{tabular}

a Scaled units, see section 3.1

${ }^{\mathrm{b}}$ Powers of ten in parentheses: $(-n) \equiv 10^{-n}$.

$\Phi^{(\mathrm{i})}\left(\xi_{-1}, \mathcal{F}\right)=\mathcal{F}^{1 / 3}\left[\mathrm{Ai}^{2}\left(\xi_{-1}\right)+\mathcal{F}^{1 / 3} \operatorname{Ai}\left(\xi_{-1}\right) \operatorname{Ai}^{\prime}\left(\xi_{-1}\right)-\frac{1}{4} \mathcal{F}^{2 / 3}\left(\operatorname{Ai}^{\prime 2}\left(\xi_{-1}\right)-\xi_{-1} \operatorname{Ai}^{2}\left(\xi_{-1}\right)\right)\right]$

Figure 11 illustrates the accuracy of various definitions for $\sigma(\mathcal{F} ; \omega)$ in equations (151), (152) and (155). As one can see, the result (155) is reasonably accurate up to $\mathcal{F} \leqslant 0.3$ and may be considered as 'exact' for such field magnitudes. Figure 12 demonstrates the contribution of different terms in (155) to $\sigma(\mathcal{F} ; \omega)$ for $\Lambda=\sqrt{2}$ and a few values of $\mathcal{F}$. As we shall show below, these contributions correspond to different levels of approximation used in previous studies of $\sigma(\mathcal{F} ; \omega)$.

\subsection{Analysis of alternative approximations}

Obviously, the formal definitions of $\alpha(\mathcal{F} ; \omega)$ and $\tilde{\boldsymbol{d}}_{\omega}$ given in (123), (128) and (130) are valid for any atom in an S-state, $\psi_{0}$, assuming that $\left|\Phi_{E}\right\rangle$ in (128) is the QS wavefunction of the initial state, $\psi_{0}$, in a static field $\mathcal{F}$ and $\mathcal{G}_{E \pm \omega}$ is the exact Green function for an atom in a static electric field plus the atomic potential (see, e.g., (97) for the case of the $\delta$-model potential). The energy $E$ is complex and involves the Stark shift and width of the initial atomic level $\left|\psi_{0}\right\rangle$. As the simplest analysis of equations (143)-(146) for the $\delta$-model potential shows, standard perturbation theory in $\mathcal{F}$ can be used only for the calculation of $\operatorname{Re} \alpha$ for below-threshold frequencies. Owing to the exponential factors in (144)-(146), $\operatorname{Im} \alpha$ for $\omega<1$ and both the real part and the imaginary part of $\alpha$ for $\omega>1$ have a non-perturbative dependence on $\mathcal{F}$ for $\mathcal{F} \rightarrow 0$. This fact corresponds to the well known asymptotic property of PT series in a static electric field and, therefore, a non-perturbative account of the static field is necessary for the approximate calculation of $\alpha(\mathcal{F} ; \omega)$ even for weak fields $\mathcal{F}$.

All the possible, non-perturbative in $\mathcal{F}$ approximations for the calculation of $\alpha(\mathcal{F} ; \omega)$ (and, obviously, of the photodetachment cross section) may be easily classified by analysing the exact expression (128) for the Fourier component $\tilde{d}_{\omega}$ of the (dual) dipole moment $\tilde{d}(t)$. The simplest approximation (I) consists in neglecting static field effects in the static field dressed state $\Phi_{E}$ and in using the Green function for a free electron in a static electric field, i.e. in using $\psi_{0}$ 

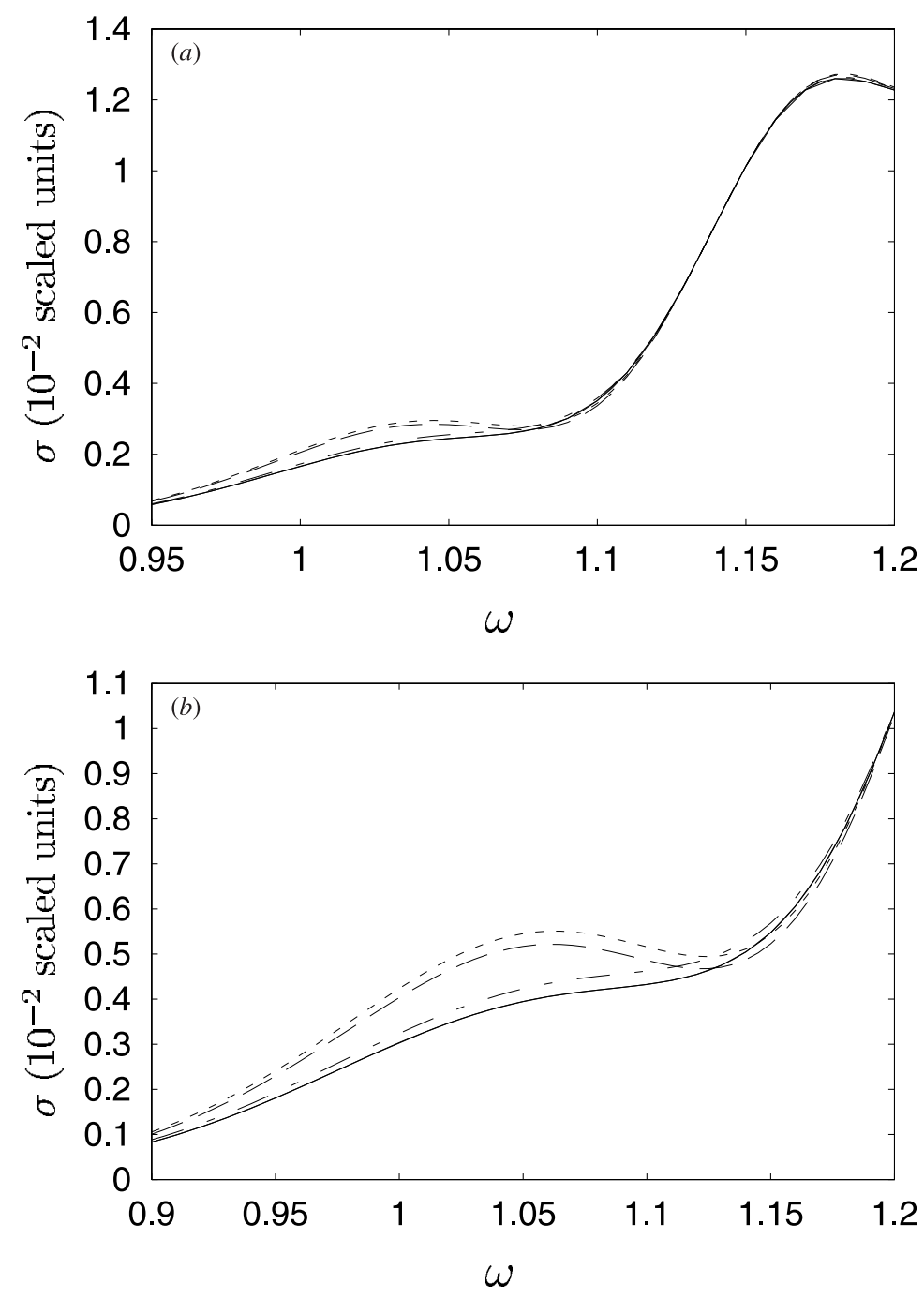

Figure 12. Contribution of different terms in (155) to the photodetachment cross section for $\Lambda=\sqrt{2}$, and for $(a) \mathcal{F}=0.015 ;(b) 0.03 ;(c) 0.06 ;(d) 0.15$. Full curve, the result (155); dotted curve, the weak-field result $\sigma^{(0)},(156)$; broken curve, the result taking into account $\sigma^{(0)}$ and $\Phi^{(i)}$ terms, (158); chain curve, the result taking into account $\sigma^{(0)}$ and $\Phi^{(r)}$ terms, (157).

and $G_{E}\left(\boldsymbol{r}, \boldsymbol{r}^{\prime}\right)$ instead of $\Phi_{E}$ and $\mathcal{G}_{E}\left(\boldsymbol{r}, \boldsymbol{r}^{\prime}\right)$. Obviously, for zero static field this approximation is equivalent to the Born approximation and in our problem (with $\mathcal{F} \neq 0$ ) may be called a static-field Born approximation. For the photodetachment calculations, this approximation has been used in $[38,41]$, although the final result in these works was obtained only approximately, in the weak-field limit (Iw; see below). A better approximation (IIa) involves, in addition to the interactions included in approximation I, an account of the distortion of the initial state by the static electric field. (For example, when calculating $\tilde{\boldsymbol{d}}_{\omega}$ using (128), approximation IIa consists in neglecting the second term on the right-hand side of (97) for $\mathcal{G}_{\mathcal{E}}$.) An alternative approximation (IIb) consists in taking account of the atomic potential in the intermediate states 

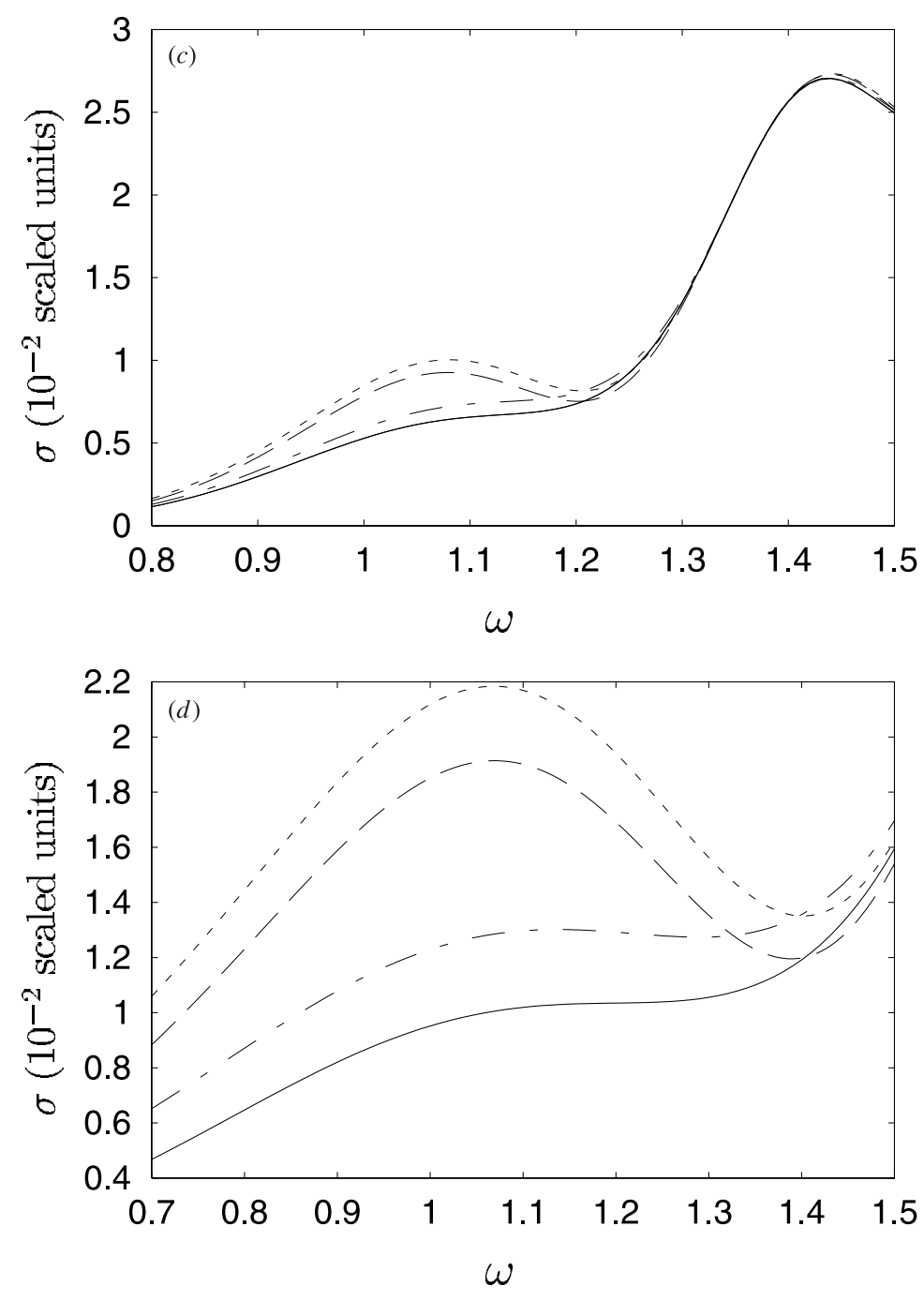

Figure 12. Continued.

(by using $\mathcal{G}_{E}$ ), but using an unperturbed initial state $\psi_{0}$. For the photodetachment calculation, this approximation is equivalent to the use of exact wavefunctions for continuum states, $\Phi_{f}(r)$, and the unperturbed initial state $\psi_{0}(\boldsymbol{r})$. The result for the photodetachment cross section in a simplified, weak-field version (IIbw) of approximation IIb was obtained in [48]. Obviously, a fourth, more exact approximation (III) consists in summing the two corrections, IIa and IIb, to approximation I. Note that III still does not provide the exact result for either $\alpha(\mathcal{F} ; \omega)$ or the photodetachment cross section since the latter quantities also include interference terms involving the corrections introduced in IIa and IIb. Table 3 below summarizes the various approximations, the interactions they include, and provides references to calculations which have employed them.

In what follows, we analyse the separate terms in (155)-(158) in order to connect them with the approximations just discussed. 
Table 3. Levels of approximation in calculating the photodetachment cross section for $\mathrm{H}^{-}$in a strong static electric field using a $\delta$-model potential and treating the laser field perturbatively.

\begin{tabular}{|c|c|c|c|c|c|c|c|}
\hline \multirow[b]{2}{*}{ Treatment } & \multicolumn{2}{|c|}{$\begin{array}{l}\text { Static electric } \\
\text { field effect }\end{array}$} & \multirow[b]{2}{*}{$\begin{array}{l}\text { Rescattering } \\
\text { effects }\end{array}$} & \multirow[b]{2}{*}{$\begin{array}{l}\text { Interference } \\
\text { effects }\end{array}$} & \multirow{2}{*}{$\begin{array}{l}\text { Transition } \\
\text { matrix } \\
\text { element }\end{array}$} & \multirow[b]{2}{*}{ Equation } & \multirow[b]{2}{*}{$\begin{array}{l}\text { Sample } \\
\text { calculation }\end{array}$} \\
\hline & $\begin{array}{l}\text { On final } \\
\text { state }\end{array}$ & $\begin{array}{l}\text { On initial } \\
\text { state }\end{array}$ & & & & & \\
\hline Iw & Yes & No & No & - & Approx. & (156) & {$[38,41]$} \\
\hline I & Yes & No & No & - & Exact & Numerical & Figure 13 \\
\hline IIa & Yes & Yes & No & - & Exact & (161) and (162) & [51] \\
\hline IIbw & Yes & No & Yes & - & Approx. & $(155)$ with $\Phi^{(i)}=0$ & [48] \\
\hline IIb & Yes & No & Yes & - & Exact & Numerical & Figure 13 \\
\hline $\mathrm{III}=\mathrm{IIa}+\mathrm{IIb}$ & Yes & Yes & Yes & No & Exact & Numerical & {$[52]$} \\
\hline Exact (for $E=E_{0}$ ) & Yes & Yes & Yes & Yes & Exact & (155) & Figures 12 and 13 \\
\hline Exact (for complex $E$ ) & Yes & Yes & Yes & Yes & Exact & (152) & Figure 12 \\
\hline
\end{tabular}

a The sample calculations are for the case of a linearly polarized laser field and a collinear static electric field. 
9.2.1. Static-field Born approximation (approximations $I$ and $I w$ ). The leading, linear in $\mathcal{F}$ term in equation (155) for $\sigma(\mathcal{F}, \omega)$ is $\sigma^{(0)}$. The result (156) for $\sigma^{(0)}$ corresponds to use of the approximation (cf (63))

$$
\Sigma\left(-E_{0}\right) \simeq M_{00}\left(-E_{0}\right)
$$

in (65), or, equivalently, to neglecting the last term (multiplying $\Lambda^{2}$ ) in (101). For the case of collinear static electric and linearly polarized laser fields, this term, $\sigma^{(0)}$, coincides with the photodetachment cross section obtained earlier by several authors [38, 41]. Although the final results in these papers involve the integrals of Airy functions, these integrals are easily calculated analytically (see appendix B) and the result for the cross section coincides with (156) for $\Lambda=1$. However, this result (156) does not correspond exactly to approximation I, but involves additional simplifications (described below) in the calculation of the amplitude appropriate for approximation I,

$$
T_{f i}^{(\mathrm{I})}=\left\langle\psi_{E}|F z| \psi_{0}\right\rangle
$$

where $\left|\psi_{E}\right\rangle$ is the free-electron wavefunction in a static electric field. The authors of [38] used a frame-transformation approximation for the final-state function $\left|\psi_{E}\right\rangle$ in (160), and in [41] the matrix element (160) was calculated using the stationary phase approximation. Both approaches are reasonable for weak enough static fields, and therefore the result can be called the weak-field approximation, Iw. It is, therefore, interesting to consider the 'exact' approximation of type I whereby the influence of the static field on the initial state is neglected, but $\left|\psi_{E}\right\rangle$ is calculated exactly as the free-electron state in a static electric field. The results for approximation I, obtained by numerical calculation of the amplitude (160), are presented in figure 13, where comparison with the weak-field approximation Iw [38,41] is also made. For high fields the more accurate approximation I gives substantially lower cross sections in the near-threshold region than the analytical result (156), i.e. the weak-field approximation Iw, which, in contrast, overestimates the exact result substantially. However, the result of approximation IIa (see below), incorporating the static-field effects in the initial state, is higher, and therefore the inaccuracy of the weak-field approximation Iw [38, 41] is not as large as it might seem from comparison with the exact approximation I. We conclude from these results that strong-field effects should be consistently included in both initial and final states.

9.2.2. Rescattering effects in IIb and IIbw approximations. The term with $\Phi^{(r)}$ in (155) can be interpreted as the result of the final-state electron-atom interaction (i.e. the so-called 'rescattering effect') of approximation IIb. The account of rescattering in this approximation was discussed in [48]. As for approximation I, the amplitude (160) with the final state taking into account the rescattering effect (i.e. using $\left|\Phi_{f}\right\rangle$ instead of $\left|\psi_{E}\right\rangle$ ) was calculated analytically only approximately, neglecting static electric field effects near $z \approx 0$. Since this approximation is valid only for a weak $\mathcal{F}$, we denote this approximate version of approximation IIb as IIbw. The resulting amplitude for this case contains the Airy functions and after the analytical calculation of the integral for the photodetachment cross section in the final equation (13) of [48] we find that the contribution of the rescattering term in approximation IIbw coincides exactly with the contribution of the term with $\Phi^{(r)}$ in (155) (including the factor with $\left|1-g_{\mathcal{F}}\right|$ in the denominator in (155)). In approximation IIb the cross section $\sigma^{\text {(IIb) }}$ again (similarly to $\left.\sigma^{(\mathrm{I})}\right)$ cannot be presented in an exact analytical form, and the result (155) with omission of the term $\Phi^{(\mathrm{i})}$ gives only the major part, $\sigma^{(\mathrm{IIbw})}$, of $\sigma^{(\mathrm{IIb})}$. In figure 13 the numerical results for $\sigma^{(\mathrm{IIb})}$ and $\sigma^{\text {(IIbw) }}$ are presented for two values of $\mathcal{F}$. One sees that the exact account of rescattering in the IIb approximation does not significantly improve the cross section compared with the 

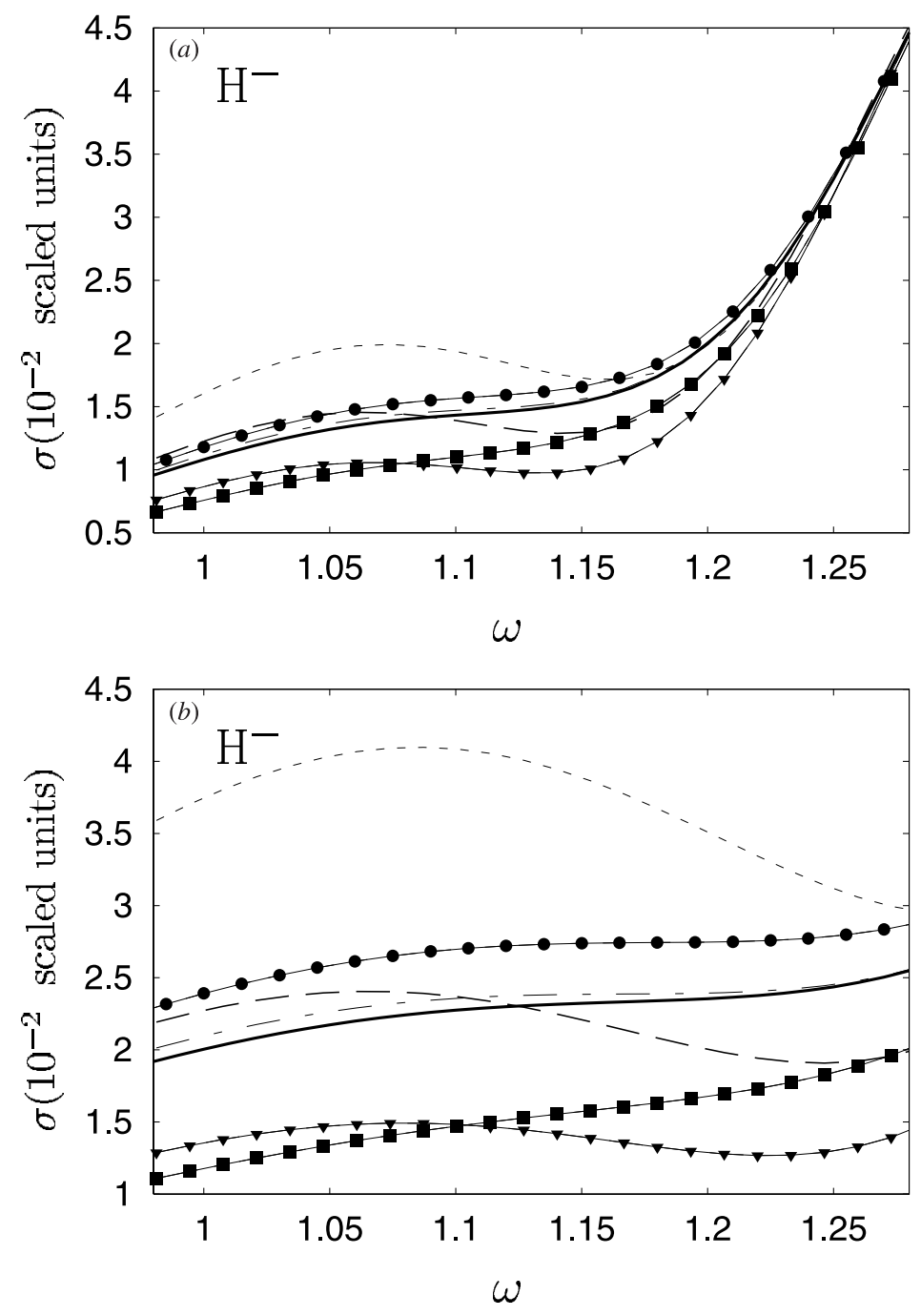

Figure 13. Comparison of different approximations for the photodetachment cross section of $\mathrm{H}^{-}$(in scaled units) for $\Lambda=\sqrt{2}$, and for $(a) \mathcal{F}=0.0429=1.44 \mathrm{MV} \mathrm{cm}^{-1}$ and $(b)$ $\mathcal{F}=0.1=3.36 \mathrm{MV} \mathrm{cm}^{-1}$. Bold full curve, the definition (151) with exact $\alpha(\mathcal{F} ; \omega)$ (see (141)) renormalized for $\mathrm{H}^{-}$. Dotted curve, the weak-field approximation Iw, $\sigma^{(0)},(156)$ (see also [38, 41]). Full curve with triangles, approximation I (our numerical result). Broken curve, approximation IIa, (161) (see also [51]). Full curve with squares, approximation IIb (our numerical result). Chain curve, approximation III (III = IIa + IIb, see also [52]). Full curve with circles, approximation IIbw, (155) with omitted term $\Phi^{(\mathrm{i})}$, see also [48]).

(exact) approximation I. On the other hand, the approximate, IIbw, account of rescattering leads to results which are much more accurate compared with the Iw case.

9.2.3. Static-field-induced distortion of the initial state. To discuss the relation of our results with approximation IIa, we note that the term with $\Phi^{(i)}$ in (155) occurs because of the exact inclusion of static field effects in both initial and final states. To check this explicitly, we have calculated the photodetachment cross section using the amplitude (153), in which the final 
state $\left|\Phi_{f}\right\rangle$ is the free electron state in a static electric field, $\left|\psi_{E}\right\rangle$, and $\left|\Phi_{E}\right\rangle$ is the QS state (77). In this calculation we used the technique developed in [24] for the integration over the radial variables in the amplitude $T_{f i}^{\text {(IIa) }}=\left\langle\psi_{E}|F z| \Phi_{E}\right\rangle$ in terms of the Airy functions. We obtain for $\sigma$ in approximation IIa (for the case of a linearly polarized field $\boldsymbol{F}(t)$ collinear to $\mathcal{F}$, i.e. $\left.\Lambda^{2}=2\right)$ :

$$
\sigma^{(\mathrm{IIa})}=\sigma^{(0)}+\sigma^{(\mathrm{i})}
$$

where

$\sigma^{(\mathrm{i})}=-\frac{32 \pi^{2} \alpha \mathcal{F}^{5 / 3}}{\omega^{3}}\left[\frac{1}{\omega} \mathrm{Ai}^{2}\left(\xi_{-1}\right)-\frac{\mathcal{F}^{2 / 3}}{\omega^{2}}\left(\mathrm{Ai}^{\prime 2}\left(\xi_{-1}\right)-\xi_{-1} \operatorname{Ai}^{2}\left(\xi_{-1}\right)\right)\right]$.

An alternative derivation of this result for $\sigma^{\text {(IIa) }}$ starts from the polarizability (128) in approximation IIa, in which only the first term, $G_{\mathcal{E}}$, is kept in the formula (97) for $\mathcal{G}_{\mathcal{E}}$. This result is

$$
\begin{aligned}
\alpha^{(\mathrm{IIa})}(\mathcal{F} ; \omega)= & -\frac{2}{\omega^{2}}-\frac{1}{g_{\mathcal{F}}^{\prime}(E) \omega^{4}}\left[\frac{4}{3} \Pi(\mathcal{F}, \omega)+\frac{2 \mathcal{F}^{2} \Lambda^{2}}{\omega}\left(g_{\mathcal{F}}^{\prime}(E+\omega)-g_{\mathcal{F}}^{\prime}(E-\omega)\right)\right. \\
& \left.+\frac{1}{2} \mathcal{F}^{2} \Lambda^{2} \sum_{n=-1}^{1} C_{2}^{n+1}\left((-1)^{n} \frac{4}{\omega^{2}} g_{\mathcal{F}}(E+n \omega)-g_{\mathcal{F}}^{\prime \prime}(E+n \omega)\right)\right] .
\end{aligned}
$$

Using the simplification (154) and taking into account only imaginary parts of the function $g_{\mathcal{F}}$ and its derivatives having the argument $E+\omega \simeq \omega-1$, and using the definition (151), we obtain for $\sigma^{\text {(IIa) }}$ once again the results (161) and (162). Moreover, equations (161) and (162) coincide exactly with the result of Gao and Starace (cf equation (64) in [51]) for the photodetachment cross section. They started with the matrix element $\left\langle\Psi_{E}|V| \psi_{0}\right\rangle$, which contains the undistorted initial state $\left|\psi_{0}\right\rangle$ but takes account of the full electron-field interaction $V$ and employs $\left|\Psi_{E}\right\rangle$, the exact wavefunction of a free electron in both the static electric and the laser fields. In fact, this function is the exact QES wavefunction of a free electron in collinear, static electric and linearly polarized laser fields. Thus, we conclude that for weak laser fields the theory of Gao and Starace [51] is equivalent to approximation IIa, i.e. to the exact account of the static electric field in the initial bound state (as well as in the final state, as in approximation I).

Comparison of (162) with (155) and (158) shows that although we have (in contrast with cases I and IIb)) the explicit form (161) and (162) for the cross section in approximation IIa, this result cannot be extracted exactly from the total cross section $\sigma$ in (155). On the other hand, we observe that this result (together with the expression (163) for $\alpha^{(\text {IIa) }}$ ) follows from the expression (68) for $\Delta \epsilon^{\mathrm{opt}}$ if we neglect non-diagonal matrix elements of $\bar{M}$ and take into account only the imaginary part of $\bar{M}_{00}\left(-E_{0}\right)$, i.e. if we approximate

$$
\bar{\Sigma}\left(-E_{0}\right) \simeq \bar{M}_{00}\left(-E_{0}\right)
$$

in the expression (69) for $\bar{\Sigma}(-E)$ (cf the approximation (159) for the calculation of $\sigma^{(0)}$ ). Thus, the rearrangement of Brillouin-Wigner series (44) for $\epsilon$ by the substitution $M_{n m} \rightarrow \bar{M}_{n m}$ is very important and allows one to account for initial-state effects, exploiting only the term $\bar{M}_{00}$. Other terms in the 'modified' series (44) (in particular, the last two terms on the right-hand side of (69)) correspond to an increasingly accurate account of rescattering in the presence of the laser field. This method is also applicable for the analysis of multiphoton effects in approximation IIa and may be used for calculations of the detachment rate in both laser and static electric strong fields [179]. 


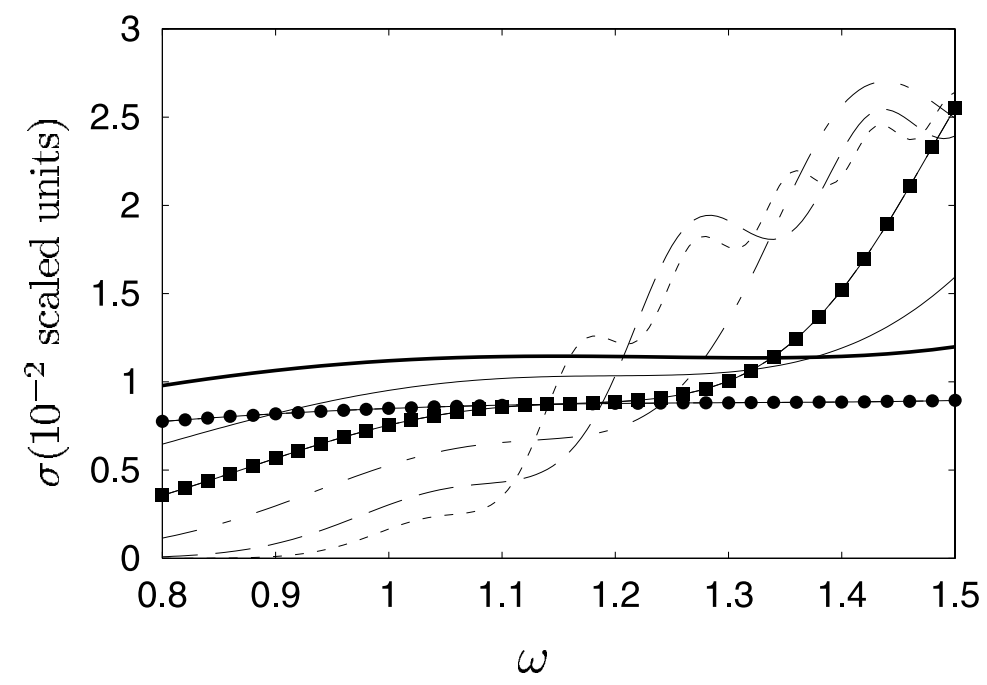

Figure 14. $\mathcal{F}$ dependence of near-threshold behaviour of the photodetachment cross section, (151), with exact $\alpha(\mathcal{F} ; \omega)$ for $\Lambda=\sqrt{2}$. Dotted curve, $\mathcal{F}=0.015$; broken curve, $\mathcal{F}=0.03$; chain curve, $\mathcal{F}=0.06$; full curve with squares, $\mathcal{F}=0.1 ;$ thin full curve, $\mathcal{F}=0.15$; bold full curve, $\mathcal{F}=0.2678$; full curve with circles, $\mathcal{F}=0.5$.

\subsection{Discussion}

9.3.1. Superposition of separately calculated effects. For the case of collinear static electric and laser fields, the above analysis justifies the separation of the result (155) into parts which have a clear physical sense (corresponding to approximations I, IIa and IIb). Further, because of the interference between different interaction pathways, the exact result cannot be presented as a simple sum of independent contributions corresponding to the terms in approximations I, IIa and IIb. Rescattering changes the contribution of static-field-induced effects in the initial state on the total cross section $\sigma$ : instead of the coefficients $\omega^{-1}$ and $\omega^{-2}$ before the Airy functions in the square brackets of equation (162), we have a joint factor $\left|1-g_{\mathcal{F}}\left(E_{0}+\omega\right)\right|^{-2}$ before the terms $\Phi^{(\mathrm{i})}$ and $\Phi^{(r)}$ in equation (155). In addition, taking simultaneous account of initialand final-state effects gives an additional ('interference') term, $\propto \mathcal{F}^{2 / 3} \operatorname{Ai}\left(\xi_{-1}\right) \mathrm{Ai}^{\prime}\left(\xi_{-1}\right)$, on the right-hand side of equation (158). At the same time, neglect of the static-field effect in the initial state removes only the term $\Phi^{(i)}$ in equation (155) and does not change the 'pure rescattering' term involving $\Phi^{(r)}$. The reason for this is the dominant role of rescattering for weak fields ( $\propto \mathcal{F}^{4 / 3}$ instead of $\propto \mathcal{F}^{5 / 3}$ for initial-state effects).

Neglecting the $\mathcal{F}$ dependence of the Airy functions, the magnitudes of the individual terms in (155) for weak fields $\mathcal{F}$ differ only by factors of $\mathcal{F}^{1 / 3}$, i.e.

$$
\sigma^{(0)} \propto \mathcal{F} \quad \sigma^{(r)} \propto \mathcal{F}^{4 / 3} \quad \sigma^{(i)} \propto \mathcal{F}^{5 / 3} .
$$

Numerically, the difference between the exact result in equation (152) and the approximations I, IIa and IIb for moderate $\mathcal{F}$ is most important for near-threshold frequencies, $(1-\Delta \omega)<$ $\omega<(1+\Delta \omega)$, where $\Delta \omega$ increases with increasing $\mathcal{F}$. With increasing $\mathcal{F}$, the frequency dependence of $\sigma$ in the near-threshold domain has a plateau-like form (see figure 14) which is most completely formed at $\mathcal{F} \sim \mathcal{F}^{(0)}=0.2678$, for which $\sigma$ achieves its maximum threshold value (cf figure 9). Figure 12 presents exact results for $\sigma$ together with results involving the $\sigma^{(0)}$ term, and, additionally, the $\Phi^{(r)}$ and $\Phi^{(i)}$ terms. It is seen that for the values of $\mathcal{F}$ considered, the term $\sigma^{(0)}$ gives the overwhelming contribution to $\sigma$ and both rescattering 


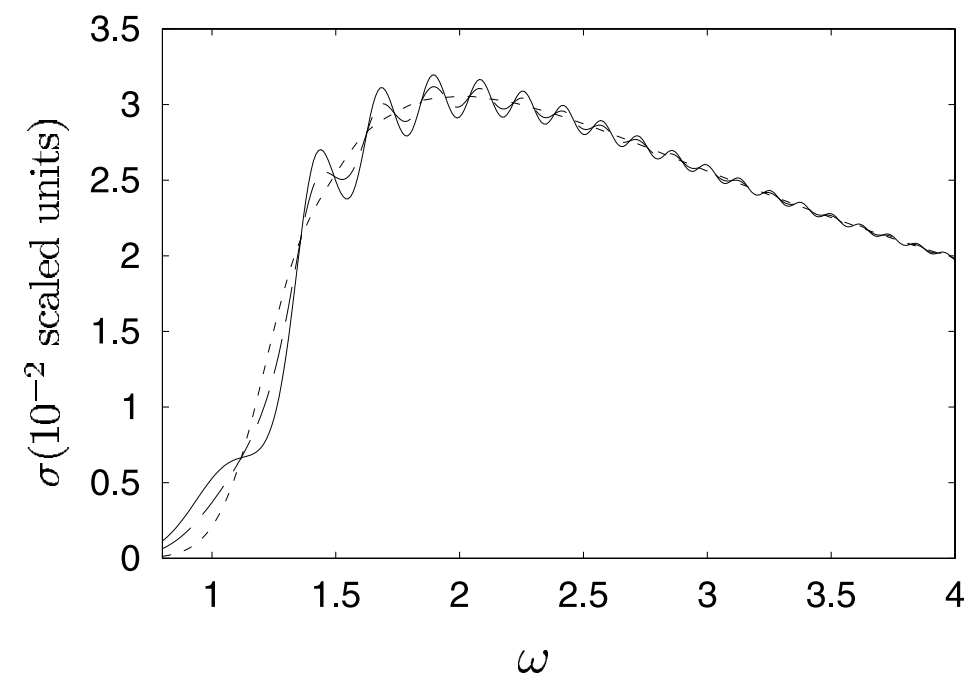

Figure 15. Frequency dependence of the photodetachment cross section (155) for $\mathcal{F}=0.06$ and different $\Lambda$. Full curve, $\Lambda=\sqrt{2}$; broken curve, $\Lambda=1$; dotted curve, $\Lambda=0$.

and initial-state effects are important only near the threshold. This fact is in contrast with the simplest estimations (165). Apparently, the suppression of the IIa and IIb terms for the case of one-photon photodetachment can be explained by the fact that rescattering effects are most important for the s-wave part of the final state, which is not populated significantly in the one-photon case because of selection rules for dipole transitions (despite the presence of the static electric field). The importance of rescattering effects in two-photon detachment was demonstrated recently in [52]. Note, that the numerical results of [52] for one-photon detachment of $\mathrm{H}^{-}$for collinear fields are very close to those obtained using equation (155), taking account of both rescattering and initial-state effects. While initially the rescattering effects in [52] were analysed only in approximation IIb, in the final equations the authors used, instead of the weak-field part of the amplitude (in approximation I), the Gao and Starace result [51], which is equivalent to an exact account of static field effects on both initial and final states. Thus, we conclude that the result in [52] is equivalent to the most exact approximation, III.

9.3.2. Interaction effects in alternative field geometries. Here we present some heuristic arguments to explain the origin of the approximate analytical expressions corresponding to approximations Iw and IIbw (equations (156) and (157)) in the exact result (155) for $\sigma$. These are especially evident for the case of orthogonal fields. In this case the oscillation pattern of the cross section vanishes (see figure 15) and equation (155) reduces to a much simpler form:

$\sigma_{\text {orth }}=\sigma_{\text {orth }}^{(0)}=\frac{32 \alpha \pi^{2} \mathcal{F}}{3 \omega^{3}}\left[\xi_{-1}^{2} \operatorname{Ai}^{2}\left(\xi_{-1}\right)-\xi_{-1} \operatorname{Ai}^{\prime 2}\left(\xi_{-1}\right)-\frac{1}{2} \operatorname{Ai}\left(\xi_{-1}\right) \operatorname{Ai}^{\prime}\left(\xi_{-1}\right)\right]$.

This expression also coincides with the result of a direct calculation of the amplitude (153) for the case of orthogonal fields [24]. Compare now $\sigma_{\text {orth }}$ with the results of the I-III calculations. Firstly, tracing the calculation of the amplitude (153), we find that the rescattering term in $\left|\Phi_{f}\right\rangle$ does not contribute to the final result because of the selection rules for the angular momentum projection $m$ for the case considered (i.e. $\Delta m= \pm 1$ ). So, equation (166) is an exact result of the IIa approximation accounting for initial-state effects. Furthermore, as for 


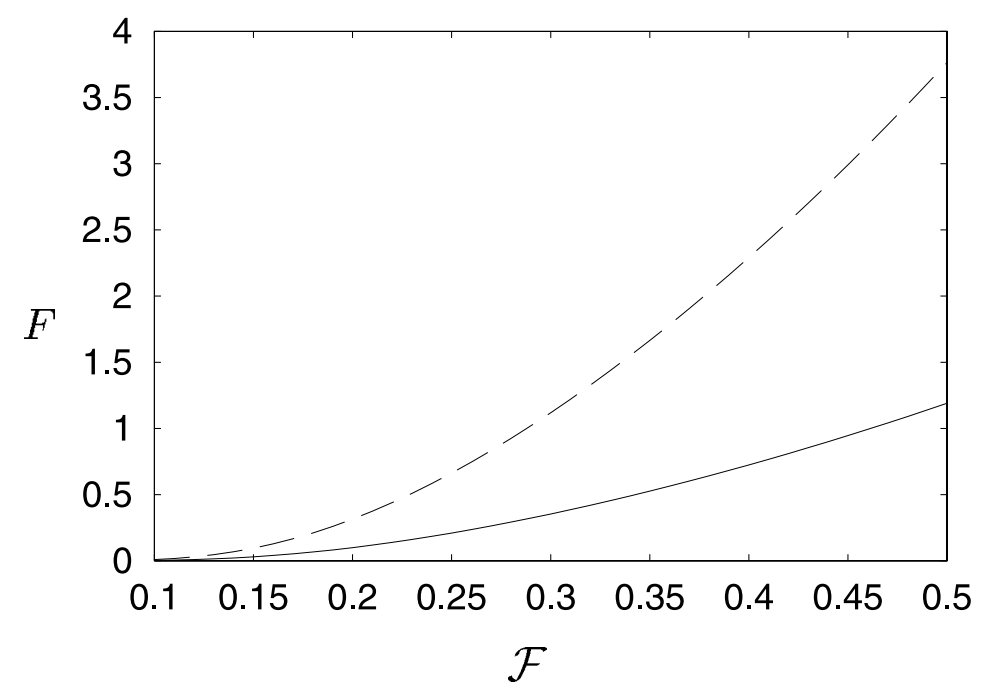

Figure 16. Static electric field dependence of the minimal amplitude of the laser field, $F_{\min }$, at which $\Gamma^{\text {stat }}=0.1 \Gamma$ (full curve) and $\Gamma^{\text {stat }}=0.01 \Gamma$ (broken curve) for the laser frequency $\omega=1.1$.

the case of collinear fields, the exact analytic calculation of the amplitude (160) for the case of approximation I is impossible; instead, we used the saddle-point approximation method for this purpose, as was done in [41]. Unexpectedly, the final result of this calculation coincides exactly with (166). Thus, a surprising situation arises, whereby an inaccuracy in the amplitude (160) is compensated exactly by an inaccuracy in its calculation! Apparently, this surprising fact (namely, the result of an approximate calculation of an approximate matrix element coincides with the exact result) merits a place among the surprises in quantum mechanics caused by the unique character of short-range potentials [180]. Mathematically, the above-discussed surprise is caused by a similarity of the momentum form, $\psi_{0}(\boldsymbol{p})$, of the unperturbed initialstate wavefunction (17) to the energy denominator in the spectral expansion of the Green function, $G_{E}\left(\boldsymbol{r}, \boldsymbol{r}^{\prime}\right)$, for the electron in a static field. (Note that this specific form of $\psi_{0}(\boldsymbol{p})$ also plays a key role in the calculation of the photodetachment amplitude taking into account the above-mentioned 'cross terms' [51].) The analysis presented shows that, firstly, rescattering effects (in contrast with initial-state effects) are sensitive to the geometry of the fields and vanish for the case $\boldsymbol{F}(t) \cdot \mathcal{F}=0$. Secondly, the analysis of the parametrization (155) in terms of contributions from the effects treated in approximations I-III is appropriate only for collinear fields. For a general geometry, the term

$$
\sigma_{\text {mix }}=-\frac{16 \pi^{2} \alpha \mathcal{F}}{3 \omega^{3}}\left(1+\frac{3}{2} \Lambda^{2}\right) \operatorname{Ai}\left(\xi_{-1}\right) \operatorname{Ai}^{\prime}\left(\xi_{-1}\right)
$$

in $\sigma^{(0)}$ describes mixed effects of approximations I and IIb. Moreover, only this part of $\sigma^{(0)}$ is important near the threshold (at $\xi_{-1}=\left(\left|E_{0}\right|-\omega\right) \mathcal{F}^{-2 / 3} \approx 0$ ) and it is this fact which gives rise to the leading term, $\propto \mathcal{F}$, in the threshold value of $\operatorname{Im} \alpha^{\text {th }}(\mathcal{F})(\operatorname{cf}(148))$.

9.3.3. Failure of the concept of photodetachment for strong static fields. Finally, we note that for a strong static field the static-field ionization width, $\Gamma_{\mathcal{F}}$, can be comparable to the light-induced width, $\Gamma \sim F^{2}$, and therefore the concept of photodetachment fails. Therefore, it is important, for a fixed value of $\mathcal{F}$, to find the minimal amplitude of a laser field, $F_{\min }$, 
for which $\Gamma_{\mathcal{F}}$ is negligible. Figure 16 presents the minimal values, $F_{\min }$, of $F$ at which the light-induced width $\Gamma$ is one and two orders of magnitude larger than $\Gamma_{\mathcal{F}}$. It is seen that, for $10 \%$ accuracy, $F_{\min }$ is of the same order as $\mathcal{F}$. In particular, for $F_{\min } \approx 0.3$ (which it seems is the maximal $F$ for which perturbation theory can be used) static field ionization can be neglected, i.e. $\Gamma_{\mathcal{F}} \leqslant 0.1 \Gamma$, for static fields $\mathcal{F} \leqslant 0.3$. The calculations were performed using (62) with an exact account of static field effects using the exact result (47) for $E$.

\section{Conclusions}

In this paper we have developed a general approach for the description of a weakly bound atomic system (e.g. a negative ion) in combined laser and static electric fields. Our analysis is based on the use of the complex quasienergy method for taking accurate account of field-induced distortion of both the energy of a bound state and its wavefunction in a quasistationary approach. It is assumed that the decay of a system (i.e. ionization or detachment) is exponential in time and that the concept of a transition rate is valid. Exact equations are derived (in section 3.2) for the quasienergy $\epsilon$ and the QQES wavefunction $\Phi_{\epsilon}(\boldsymbol{r}, t)$ of a weakly bound level (in a $\delta$-model potential) for a general (i.e. elliptical) laser-field polarization and for an arbitrary geometry of the two fields. Our results recover the results of previous studies carried out for a number of special cases. They are also effective tools for calculations that are non-perturbative in both fields as well as for calculations that account for the laser field perturbatively for the case of a strong static electric field. The special case of static-field-induced ionization is analysed in detail and simple analytical approximations for the level shift (51) and the width (52) are obtained. Both of these approximations are highly accurate up to $\mathcal{F} \leqslant 1$.

We have also addressed two general aspects of strong field-atom interactions. (a) The normalization procedure for wavefunctions of the QS (resonance) state in a strong electric field and, more generally, the QQES, which is important for the development of time-dependent perturbation theory using a QS or QQES basis; (b) the concept of the dynamic polarizability and the field-induced dipole moment for a decaying atomic system (in a static electric field and/or in a strong laser field). We have shown that a proper normalization of QQES states may be achieved by introducing 'dual' functions, $\tilde{\Phi}_{\epsilon}(\boldsymbol{r}, t)$, analogous to the case of resonance states for radiationless problems in atomic and nuclear physics. Based on the concept of dual functions, we introduced the dipole moment of an atom in a strong field, $\tilde{\boldsymbol{d}}(t)$, which is similar (but not identical) to the standard dipole moment $\boldsymbol{d}(t)$ (i.e. the expectation value of operator $\hat{\boldsymbol{d}}$ ) of a stable system. In this way, Fourier components of $\tilde{d}(t)$ and the corresponding polarizabilities are connected with amplitudes for an observable effect, photon scattering, rather than with the (unobserved) distortion of the charge density, as is the case for standard definitions for a stable quantum system. For a negative ion, this formulation is illustrated analytically (in sections 6 and 7 ) for the exactly solvable $\delta$-potential model.

The major part of the analytical and numerical results presented in this review is related to our extensive analysis of the linear-in-laser-intensity corrections for the complex quasienergy in both laser and (possibly strong) static electric fields. The results for a weak laser field are presented in terms of the static field dependence and the laser frequency dependence of the dynamic polarizability of the system, whose real part gives the shift of the energy level due to the external fields and whose imaginary part gives the decay width. For not too strong static fields, the decay width reduces to that for the photodetachment cross section. The analysis of the imaginary part of the polarizability for different levels of approximation allows us to recover results of previous studies of photodetachment in the presence of an external static field: the weak-field approximation [38,41], treatments for strong static-field effects [51], treatments of the rescattering effect [48] and recent calculations [52] employing the $S$-matrix method. The 
advantage of the present approach is that all effects are treated on an equal footing, and the final results are presented in a unified analytical form. Although for conciseness we have limited the considerations of this paper to only one-photon effects, the present quasienergy approach can be easily used to calculate higher-order (multiphoton) processes analytically.

\section{Acknowledgments}

This work has been supported in part by the grant programme 'Universities of Russia' and the Russian Foundation for Basic Research under grant no 00-02-17843 (NLM and MVF), and by the US National Science Foundation under grant nos PHY-9722110 (AFS) and PHY-980187 (IIF).

\section{Appendix A. Green functions for a free electron in static electric and laser fields}

- In the length gauge for the electron-field interaction the Lagrangian has the following form:

$$
L(\boldsymbol{r}, \dot{\boldsymbol{r}}, t)=\frac{1}{2} m \dot{\boldsymbol{r}}^{2}-V(\boldsymbol{r}, t)
$$

where

$$
V(\boldsymbol{r}, t)=|e|(\boldsymbol{F}(t)+\mathcal{F}) \cdot \boldsymbol{r}
$$

and where $\boldsymbol{F}(t)$ describes the time-dependent laser field and $\mathcal{F}$ the static electric field. We define the Green function by the following equation:

$\left(\mathrm{i} \hbar \frac{\partial}{\partial t}+\frac{\hbar^{2}}{2 m} \nabla_{r}^{2}-V(\boldsymbol{r}, t)\right) G^{( \pm)}\left(\boldsymbol{r}, t ; \boldsymbol{r}^{\prime}, t^{\prime}\right)=\delta\left(\boldsymbol{r}-\boldsymbol{r}^{\prime}\right) \delta\left(t-t^{\prime}\right)$

where $(+)$ (or $(-))$ corresponds to the retarded (or advanced) Green function. Feynman's form of the Green function is

$G^{( \pm)}\left(\boldsymbol{r}, t ; \boldsymbol{r}^{\prime}, t^{\prime}\right)=\mp \frac{\mathrm{i}}{\hbar}\left(\frac{m}{2 \pi \mathrm{i} \hbar\left(t-t^{\prime}\right)}\right)^{3 / 2} \Theta\left[ \pm\left(t-t^{\prime}\right)\right] \mathrm{e}^{\frac{\mathrm{i}}{\hbar} S\left(\boldsymbol{r}, t ; \boldsymbol{r}^{\prime}, t\right)}$

where $S$ is the classical action, which for the case considered can be presented as $S\left(\boldsymbol{r}, t ; \boldsymbol{r}^{\prime}, t\right)=T\left(\boldsymbol{r}, t ; \boldsymbol{r}^{\prime}, t^{\prime}\right)+M\left(t, t^{\prime}\right)+R\left(\boldsymbol{r}, t ; \boldsymbol{r}^{\prime}, t^{\prime}\right)$

$$
+L\left(t, t^{\prime}\right)+K\left(\boldsymbol{r}, t ; \boldsymbol{r}^{\prime}, t^{\prime}\right)+P\left(t, t^{\prime}\right)
$$

where

$$
\begin{aligned}
& T\left(\boldsymbol{r}, t ; \boldsymbol{r}^{\prime}, t^{\prime}\right)=\frac{m\left(\boldsymbol{r}-\boldsymbol{r}^{\prime}\right)^{2}}{2\left(t-t^{\prime}\right)} \\
& M\left(t, t^{\prime}\right)=-\frac{e^{2}}{2 m c^{2}} \int_{t^{\prime}}^{t} \boldsymbol{A}(\tau)^{2} \mathrm{~d} \tau+\frac{e^{2}}{2 m c^{2}\left(t-t^{\prime}\right)}\left(\int_{t^{\prime}}^{t} \boldsymbol{A}(\tau) \mathrm{d} \tau\right)^{2} \\
& R\left(\boldsymbol{r}, t ; \boldsymbol{r}^{\prime}, t^{\prime}\right)=-\frac{|e|\left(\boldsymbol{r}-\boldsymbol{r}^{\prime}\right)}{c\left(t-t^{\prime}\right)} \int_{t^{\prime}}^{t} \boldsymbol{A}(\tau) \mathrm{d} \tau+\frac{|e|}{c}\left(\boldsymbol{r} \cdot \boldsymbol{A}(t)-\boldsymbol{r}^{\prime} \cdot \boldsymbol{A}\left(t^{\prime}\right)\right) \\
& L\left(t, t^{\prime}\right)=-\frac{e^{2} \mathcal{F}^{2}\left(t-t^{\prime}\right)^{3}}{24 m} \\
& K\left(\boldsymbol{r}, t ; \boldsymbol{r}^{\prime}, t^{\prime}\right)=-\frac{1}{2}|e| \mathcal{F} \cdot\left(\boldsymbol{r}+\boldsymbol{r}^{\prime}\right)\left(t-t^{\prime}\right) \\
& P\left(t, t^{\prime}\right)=\frac{e^{2}}{2 m c} \mathcal{F}\left[2 \int_{t^{\prime}}^{t} \boldsymbol{A}(\tau) \tau \mathrm{d} \tau-\left(t+t^{\prime}\right) \int_{t^{\prime}}^{t} \boldsymbol{A}(\tau) \mathrm{d} \tau\right]
\end{aligned}
$$

where $\boldsymbol{A}(t)$ is the vector potential of the laser field, i.e. $\boldsymbol{F}(t)=-\partial \boldsymbol{A}(t) / c \partial t$. 
- In the velocity gauge for the electron-field interaction the equation for $G^{( \pm)}$is

$$
\left(\mathrm{i} \hbar \frac{\partial}{\partial t}-\frac{1}{2 m}\left[\hat{\boldsymbol{p}}+\frac{|e|}{c} \boldsymbol{A}(t)\right]^{2}-|e| \mathcal{F} \cdot \boldsymbol{r}\right) G^{( \pm)}\left(\boldsymbol{r}, t ; \boldsymbol{r}^{\prime}, t^{\prime}\right)=\delta\left(\boldsymbol{r}-\boldsymbol{r}^{\prime}\right) \delta\left(t-t^{\prime}\right) .
$$

Here equations (A3) and (A4) are again valid with the exception that the term $R$ has the reduced form

$$
R\left(\boldsymbol{r}, t ; \boldsymbol{r}^{\prime}, t^{\prime}\right)=-\frac{|e|\left(\boldsymbol{r}-\boldsymbol{r}^{\prime}\right)}{c\left(t-t^{\prime}\right)} \int_{t^{\prime}}^{t} \boldsymbol{A}(\tau) \mathrm{d} \tau
$$

- We present below the explicit form of the Green functions (A3) in the length gauge for an elliptically polarized monochromatic field with an electric vector

$$
\boldsymbol{F}(t)=F \operatorname{Re}\left\{e \mathrm{e}^{-\mathrm{i} \omega t}\right\}
$$

where $e$ is the polarization vector

$$
e=\frac{\hat{\epsilon}+\mathrm{i} \eta[\hat{k} \times \hat{\epsilon}]}{\sqrt{1+\eta^{2}}} \quad e \cdot e^{*}=1 \quad-1 \leqslant \eta \leqslant+1 .
$$

The geometry of the static field is determined by the angles $\theta, \phi$,

$$
\mathcal{F}=\mathcal{F}\{\sin \theta \cos \phi, \sin \theta \sin \phi, \cos \theta\}
$$

where

$$
\left\{c_{1}, c_{2}, c_{3}\right\} \equiv \hat{\epsilon} c_{1}+[\hat{k} \times \hat{\epsilon}] c_{2}+\hat{k} c_{3}
$$

and where $\hat{k}$ and $\hat{\epsilon}$ determine the directions of the photon beam and the major axis of the polarization ellipse, respectively.

The Green functions in scaled units (section 3.1) are

$$
\begin{aligned}
G^{( \pm)}\left(\boldsymbol{r}, t ; \boldsymbol{r}^{\prime}, t^{\prime}\right) & =G_{ \pm}^{(0)}\left(\boldsymbol{r}-\boldsymbol{r}^{\prime}, t-t^{\prime}\right) \\
\times & \exp \left\{\mathrm{i}\left[M\left(t, t^{\prime}\right)+R\left(\boldsymbol{r}, t ; \boldsymbol{r}^{\prime}, t^{\prime}\right)+L\left(t, t^{\prime}\right)+K\left(\boldsymbol{r}, t ; \boldsymbol{r}^{\prime}, t^{\prime}\right)+P\left(t, t^{\prime}\right)\right]\right\}
\end{aligned}
$$

where

$G_{ \pm}^{(0)}\left(\boldsymbol{r}-\boldsymbol{r}^{\prime}, \tau\right)=\mp \frac{\mathrm{i}}{(4 \pi \mathrm{i} \tau)^{3 / 2}} \Theta( \pm \tau) \exp \left[\mathrm{i} \frac{\left(\boldsymbol{r}-\boldsymbol{r}^{\prime}\right)^{2}}{4 \tau}\right] \quad \tau=t-t^{\prime}$

are the Green functions for a free electron, and where

$$
\begin{aligned}
& M\left(t, t^{\prime}\right)=-\frac{\gamma^{2}}{\omega}\left[\omega \tau\left(1-\frac{4 \sin ^{2}(\omega \tau / 2)}{(\omega \tau)^{2}}\right)-l \cos \omega(2 t-\tau)\left(\sin \omega \tau-\frac{4 \sin ^{2}(\omega \tau / 2)}{\omega \tau}\right)\right] \\
& R\left(\boldsymbol{r}, t ; \boldsymbol{r}^{\prime}, t^{\prime}\right)=-\frac{\sqrt{2} \gamma}{\sqrt{1+\eta^{2}}}\left[\frac{2}{\omega \tau} \sin (\omega \tau / 2)\left(\boldsymbol{r}-\boldsymbol{r}^{\prime}\right)\{-\sin \omega(t-\tau / 2), \eta \cos \omega(t-\tau / 2), 0\}\right. \\
& \left.-\boldsymbol{r}\{-\sin \omega t, \eta \cos \omega t, 0\}+\boldsymbol{r}^{\prime}\left\{-\sin \omega t^{\prime}, \eta \cos \omega t^{\prime}, 0\right\}\right] \\
& L\left(t, t^{\prime}\right)=-\frac{1}{12} \mathcal{F}^{2} \tau^{3} \\
& K\left(\boldsymbol{r}, t ; \boldsymbol{r}^{\prime}, t^{\prime}\right)=-\frac{1}{2} \mathcal{F} \cdot\left(\boldsymbol{r}+\boldsymbol{r}^{\prime}\right) \tau \\
& P\left(t, t^{\prime}\right)=\Lambda \frac{4 \gamma \mathcal{F}}{\omega^{2}}\left(\frac{1}{2} \omega \tau \cos \frac{1}{2} \omega \tau-\sin \frac{1}{2} \omega \tau\right) \cos \left(\omega(t-\tau / 2)-\phi_{1}\right) .
\end{aligned}
$$


In the above equations we use the following notation:

$$
\gamma^{2}=\frac{F^{2}}{2 \omega^{2}} \quad l=\frac{1-\eta^{2}}{1+\eta^{2}} \quad \Lambda=\sin \theta \sqrt{1+l \cos 2 \phi} .
$$

The angle $\phi_{1}$ is determined as follows:

$$
\tan \phi_{1}=\eta \tan \phi \quad \phi_{1}=\arg (e \cdot \mathcal{F}) .
$$

\section{Appendix B. Calculation and regularization of some integrals}

In this appendix we calculate some integrals which are necessary in the analysis of higher orders of perturbation theory in the laser field, taking into account the static electric field exactly.

- Firstly, we consider the calculation of the integrals

$$
T=\int_{0}^{\infty} \frac{\mathrm{e}^{\mathrm{i}\left(\varepsilon \tau-\frac{1}{12} \mathcal{F}^{2} \tau^{3}\right)}}{\tau^{1 / 2}} \mathrm{~d} \tau \quad M=\int_{0}^{\infty} \frac{\mathrm{e}^{\mathrm{i}\left(\varepsilon \tau-\frac{1}{12} \mathcal{F}^{2} \tau^{3}\right)}-1}{\tau^{3 / 2}} \mathrm{~d} \tau
$$

using a technique similar to that developed in [52], using the explicit form for the Green function, $G_{\varepsilon}\left(\boldsymbol{r}, \boldsymbol{r}^{\prime}\right)$, of an electron with energy $\varepsilon$ in a static electric field, $\mathcal{F}$ [181]. The (retarded) Green function for an electron in a static field is (see (14)):

$G\left(\boldsymbol{r}, t ; \boldsymbol{r}^{\prime}=0, t^{\prime}\right)=-\frac{\mathrm{i} \Theta(\tau)}{(4 \pi \mathrm{i} \tau)^{3 / 2}} \exp \left[\mathrm{i}\left(\frac{r^{2}}{4 \tau}-\frac{\mathcal{F} \cdot \boldsymbol{r} \tau}{2}-\frac{\mathcal{F}^{2} \tau^{3}}{12}\right)\right]$

where $\tau=t-t^{\prime}$. The function $G_{\varepsilon}(\boldsymbol{r}) \equiv G_{\varepsilon}\left(\boldsymbol{r}, \boldsymbol{r}^{\prime}=0\right)$ is the Fourier component of $G\left(\boldsymbol{r}, t ; \boldsymbol{r}^{\prime}=0, t^{\prime}\right)$ and can be presented as [181]

$G_{\varepsilon}(\boldsymbol{r})=\int_{0}^{\infty} \mathrm{e}^{\mathrm{i} \varepsilon \tau} G\left(\boldsymbol{r}, \tau ; \boldsymbol{r}^{\prime}=0, t^{\prime}=0\right) \mathrm{d} \tau=\frac{1}{4 r}\left[\operatorname{Ai}^{\prime}\left(Z_{1}\right) \operatorname{Ci}\left(Z_{2}\right)-\operatorname{Ai}\left(Z_{1}\right) \operatorname{Ci}^{\prime}\left(Z_{2}\right)\right]$

where $\mathcal{F}$ is directed along the $z$-axis, $Z_{1}=\xi+\mathcal{F}^{1 / 3}(z+r) / 2, Z_{2}=\xi+\mathcal{F}^{1 / 3}(z-r) / 2$, $\xi=-\varepsilon \mathcal{F}^{-2 / 3} \cdot \operatorname{Ai}(\xi), \operatorname{Bi}(\xi)$ and $\operatorname{Ci}(\xi)=\operatorname{Bi}(\xi)+\mathrm{i} \operatorname{Ai}(\xi)$ are regular and irregular Airy functions [156].

Direct differentiation of $G_{\varepsilon}(r)$ gives

$$
\frac{\partial}{\partial \varepsilon} G_{\varepsilon}(r)=-\frac{1}{4 \mathcal{F}^{1 / 3}} \operatorname{Ai}\left(Z_{1}\right) \operatorname{Ci}\left(Z_{2}\right)
$$

In the limiting case $r \rightarrow 0$, the first derivative with respect to $\epsilon$ of the integral over $\tau$ in (B3) differs from the integral $T$ only by a constant factor, i.e.

$$
T=(4 \pi \mathrm{i})^{3 / 2} \lim _{r \rightarrow 0} \frac{\partial}{\partial \varepsilon} G_{\varepsilon}(r)=-\frac{(2 \pi \mathrm{i})^{3 / 2}}{\sqrt{2} \mathcal{F}^{1 / 3}} I(\xi)
$$

where we have introduced the function $I(\xi)=\operatorname{Ai}(\xi) \operatorname{Ci}(\xi)$. The integral $M$ may be calculated by an integration by parts using (B5). The result is

$$
M=-\frac{\mathrm{i}}{\sqrt{2}}(2 \pi \mathrm{i})^{3 / 2} \mathcal{F}^{1 / 3} J(\xi)
$$

where $J(\xi)=\operatorname{Ai}^{\prime}(\xi) \mathrm{Ci}^{\prime}(\xi)-\xi \operatorname{Ai}(\xi) \mathrm{Ci}(\xi)$. From (B1) one may obtain the following formal identity:

$$
\frac{\partial}{\partial \varepsilon} M=\mathrm{i} T
$$

which is equivalent to the relation $J^{\prime}(\xi)=-I(\xi)$, which can be verified by direct differentiation of the Airy functions. 
- Let us consider now the general integral of the kind

$$
M_{n}(\varepsilon)=\int_{0}^{\infty} \frac{\mathrm{e}^{\mathrm{i}\left(\varepsilon \tau-\frac{1}{12} \mathcal{F}^{2} \tau^{3}\right)} g(\tau)}{\tau^{1 / 2+n}} \mathrm{~d} \tau \quad n \geqslant 2
$$

which may have (depending on the function $g(\tau)$ ) a singularity at the lower limit. The necessary conditions for the convergence of this integral are

$$
\begin{aligned}
& \left.\frac{\partial^{k}}{\partial t^{k}} g(t)\right|_{t \rightarrow 0} \rightarrow t^{n-k} \rightarrow 0 \quad k=0, \ldots, n \\
& \left.\frac{\partial^{k}}{\partial t^{k}} g(t)\right|_{t \rightarrow \infty} \rightarrow \text { constant. }
\end{aligned}
$$

Let $g(t)$ be a periodic function,

$$
g(t)=\sum_{m} a_{m} \mathrm{e}^{\mathrm{i} m \omega t}
$$

Then the condition (B9) has the form

$$
\sum_{m} a_{m} m^{k}=0
$$

The conditions (B9)-(B11) show that upon integrating the integral in (B8) $k$ times $(k<n)$ by parts, the sum of all terms arising from the values of the successive integrands at the lower and upper limits equals zero. Therefore, relation (B11) allows us to present the convergent integral (B8) as the sum of formally divergent integrals $\tilde{M}_{m}(\varepsilon)$, where

$$
\begin{aligned}
& M_{n}(\varepsilon)=\sum_{m} \tilde{M}_{m}(\varepsilon+m \omega) a_{m} \\
& \tilde{M}_{m}(\varepsilon)=\int_{0}^{\infty} \frac{\mathrm{e}^{\mathrm{i}\left(\varepsilon \tau-\frac{1}{12} \mathcal{F}^{2} \tau^{3}\right)}}{\tau^{1 / 2+m}} \mathrm{~d} \tau .
\end{aligned}
$$

Moreover, despite the formal divergence of (B12), this integral can be calculated by successive integration by parts until the parameter $n$ in the denominator of the last integrand equals zero. Since the singularities at the lower limit are cancelled in accordance with the condition (B11), they can be dropped in the calculation of $\tilde{M}_{m}(\varepsilon)$. Obviously, this suggested procedure of regularization can be used only if the integrals $\tilde{M}_{m}(\varepsilon)$ arise in the intermediate steps of the calculation of a convergent integral of the type (B8).

Integrating relation (B12) by parts, we obtain the following recurrence relation for $\tilde{M}_{n}(\varepsilon)$ :

$$
\tilde{M}_{n}(\varepsilon)=\frac{\mathrm{i}}{n-\frac{1}{2}}\left[\varepsilon \tilde{M}_{n-1}(\varepsilon)+\frac{\mathcal{F}^{2}}{4} \frac{\partial^{2}}{\partial \varepsilon^{2}} \tilde{M}_{n-1}(\varepsilon)\right] .
$$

As an example, we present the explicit form for $\tilde{M}_{2}(\varepsilon)$ and $\tilde{M}_{3}(\varepsilon)$, calculated using (B13) and the explicit form (B6) for $\tilde{M}_{1}(\varepsilon)=M$ :

$$
\begin{aligned}
& \tilde{M}_{2}(\varepsilon)=-\sqrt{2} \frac{1}{3}(2 \pi \mathrm{i})^{3 / 2} \mathcal{F}\left[\xi J(\xi)+\frac{1}{4} I^{\prime}(\xi)\right] \\
& \tilde{M}_{3}(\varepsilon)=\mathrm{i} \sqrt{2} \frac{1}{15}(4 \pi \mathrm{i})^{3 / 2} \mathcal{F}^{5 / 3}\left[\xi^{2} J(\xi)+\frac{1}{4} \xi I^{\prime}(\xi)+\frac{3}{8} I(\xi)\right] .
\end{aligned}
$$


- In calculations of photodetachment cross sections there arise integrals of the product of two Airy functions

$$
\int_{a}^{b} \mathrm{~d} x x^{n} \mathrm{Ai}^{2}(x) \quad \text { or } \quad \int_{a}^{b} \mathrm{~d} x x^{n}\left(\mathrm{Ai}^{\prime}(x)\right)^{2} \quad n=0,1,2 .
$$

Although a more general form of the integral involving a product of Airy functions can be used in such calculations (see, e.g., $[16,24]$ ), a simpler way is to use the well known differential equation for the product $w(x)=f_{1}(x) f_{2}(x)$ of two arbitrary solutions of the Airy equation (see, e.g., [156]),

$$
w^{\prime \prime \prime}-4 x w^{\prime}-2 w=0
$$

Namely, integrating this equation we obtain for the case $f_{1}=f_{2}=\operatorname{Ai}(x)$

$$
\int_{a}^{b} \mathrm{~d} x \operatorname{Ai}^{2}(x)=\left.\left(x \operatorname{Ai}^{2}(x)-\operatorname{Ai}^{\prime 2}(x)\right)\right|_{a} ^{b} .
$$

Integration of equation (B16) multiplied by $x$ allows us to find also the second integral,

$$
\int \mathrm{d} x x \operatorname{Ai}^{2}(x)=\frac{1}{3}\left(x^{2} \operatorname{Ai}^{2}(x)-x \operatorname{Ai}^{\prime 2}(x)+\operatorname{Ai}(x) \operatorname{Ai}^{\prime}(x)\right)
$$

which is necessary for analytical calculations of the integrals which enter the photodetachment cross sections in the Iw, IIa and IIbw approximations.

\section{Appendix C. Limiting expressions for the regularized Green function $g_{\mathcal{F}}(E)$ and its}

\section{derivatives}

We have

$$
g_{\mathcal{F}}(E)=-\pi \mathcal{F}^{1 / 3} J\left(-E / \mathcal{F}^{2 / 3}\right)
$$

where

$$
J(\xi)=\operatorname{Ai}^{\prime}(\xi) \mathrm{Ci}^{\prime}(\xi)-\xi \operatorname{Ai}(\xi) \operatorname{Ci}(\xi)
$$

and where $\operatorname{Ci}(\xi)=\operatorname{Bi}(\xi)+\mathrm{i} \operatorname{Ai}(\xi)$. The derivatives of $g_{\mathcal{F}}(E)$ are

$$
\begin{aligned}
& g_{\mathcal{F}}^{\prime}(E)=\frac{\partial}{\partial E} g_{\mathcal{F}}(E)=-\pi \mathcal{F}^{-1 / 3} I(\xi)=-\pi \mathcal{F}^{-1 / 3} \operatorname{Ai}(\xi) \mathrm{Ci}(\xi) \\
& g_{\mathcal{F}}^{\prime \prime}(E)=\frac{\partial^{2}}{\partial E^{2}} g_{\mathcal{F}}(E)=\frac{\pi}{\mathcal{F}} \frac{\partial I(\xi)}{\partial \xi}=\frac{\pi}{\mathcal{F}}\left(\mathrm{Ai}^{\prime}(\xi) \mathrm{Ci}(\xi)+\operatorname{Ai}(\xi) \mathrm{Ci}^{\prime}(\xi)\right) .
\end{aligned}
$$

Obviously, higher-order derivatives may be evaluated, as described above, using the Airy equation. The limiting expressions for these functions for cases of large and small arguments can be obtained using well known results for Airy functions [156].

- The asymptotic expansions for large positive $\xi=-E / \mathcal{F}^{2 / 3}$ are:

$$
\begin{aligned}
& \pi J(\xi) \rightarrow-\sqrt{\xi}\left(1-\frac{1}{32 \xi^{3}}\right)+\frac{\mathrm{i}}{8 \xi}\left(1-\frac{17}{24 \xi^{3 / 2}}\right) \mathrm{e}^{-\frac{4}{3} \xi^{3 / 2}} \\
& \pi I(\xi) \rightarrow \frac{1}{2 \sqrt{\xi}}\left(1+\frac{5}{32 \xi^{3}}\right)+\frac{\mathrm{i}}{4 \sqrt{\xi}}\left(1-\frac{5}{24 \xi^{3 / 2}}\right) \mathrm{e}^{-\frac{4}{3} \xi^{3 / 2}} \\
& \pi I^{\prime}(\xi) \rightarrow-\frac{1}{4 \xi^{3 / 2}}\left(1+\frac{35}{32 \xi^{3}}\right)-\frac{\mathrm{i}}{2}\left(1+\frac{1}{24 \xi^{3 / 2}}\right) \mathrm{e}^{-\frac{4}{3} \xi^{3 / 2}} .
\end{aligned}
$$

The second terms in parentheses allow us to estimate the accuracy of these (two-term) asymptotic expansions. 
- The asymptotics for large negative arguments $\xi(\xi \rightarrow-\infty)$ are:

$$
\begin{aligned}
& \pi J(\xi) \rightarrow-\frac{1}{4 \xi} \sin x-\frac{17}{96(-\xi)^{5 / 2}} \cos x+\mathrm{i}\left(\sqrt{-\xi}+\frac{1}{4 \xi} \cos x+\frac{17}{96(-\xi)^{5 / 2}} \sin x\right) \\
& \pi I(\xi) \rightarrow \frac{1}{2 \sqrt{-\xi}} \cos x+\frac{5}{48 \xi^{2}} \sin x+\mathrm{i}\left(\frac{1}{2 \sqrt{-\xi}}(1+\sin x)-\frac{5}{48(-\xi)^{3 / 2}} \cos x\right) \\
& \pi I^{\prime}(\xi) \rightarrow \sin x+\frac{1}{24(-\xi)^{3 / 2}} \cos x-\mathrm{i}\left(\cos x-\frac{1}{24(-\xi)^{3 / 2}}(6+\sin x)\right)
\end{aligned}
$$

where

$$
x=\frac{4}{3}(-\xi)^{3 / 2} .
$$

- The expansions for $\xi \approx 0$ (valid for both positive and negative $\xi$ ) are:

$$
\begin{aligned}
& \pi J(\xi)=\frac{1}{2 c_{0}^{2}}\left[-c_{0}^{3}-x+\frac{1}{3} x^{2}+\frac{\mathrm{i}}{\sqrt{3}}\left(c_{0}^{3}-x+x^{2}\right)\right] \\
& \pi I(\xi)=\frac{1}{2 c_{0}}\left[1-x^{2}+\frac{1}{3 c_{0}^{3}} x^{3}+\frac{\mathrm{i}}{\sqrt{3}}\left(1-2 x+x^{2}\right)\right] \\
& \pi I^{\prime}(\xi)=-x+\frac{1}{2 c_{0}^{3}} x^{2}-\frac{\mathrm{i}}{\sqrt{3}}(1-x)
\end{aligned}
$$

where

$$
x=c_{0} \xi \quad c_{0}=\frac{1}{2 \pi} 3^{5 / 6}\left[\Gamma\left(\frac{2}{3}\right)\right]^{2}=0.729011
$$

and $\Gamma(x)$ is the gamma function.

\section{References}

[1] Dodonov V V and Man'ko V I 1989 Invariants and the evolution of nonstationary quantum systems Proc. Lebedev Phys. Inst. Acad. Sci. USSR vol 183, ed M A Markov (Commack, NY: Nova)

Dodonov V V, Man'ko V I and Nikonov D E 1992 Phys. Lett. A 162359

[2] Kleber M 1994 Phys. Rep. 236331

[3] Demkov Yu N and Ostrovsky V N 1988 Zero-Range Potentials and Their Applications in Atomic Physics (New York: Plenum)

[4] Albeverio S, Gesztesy F, Hoegh-Krön and Holden H 1988 Solvable Models in Quantum Mechanics (Berlin: Springer)

[5] Zel'dovich Ya B 1966 Zh. Eksp. Teor. Fiz. 511492 (Engl. transl. 1967 Sov. Phys.-JETP 24 1006)

[6] Manakov N L, Ovsiannikov V D and Rapoport L P 1986 Phys. Rep. 141319

[7] Bergeman T et al 1984 Phys. Rev. Lett. 53775

[8] Smith W W et al 1985 Atomic Excitation and Recombination in External Fields ed M H Nayfeh and C W Clark (New York: Gordon and Breach) p 211

[9] Bryant H C et al 1983 Phys. Rev. A 272889

[10] Keldysh L V 1958 Zh. Eksp. Teor. Fiz. 341138 (Engl. transl. Sov. Phys.-JETP 34 788)

[11] Franz W 1958 Z. Naturf. A 13484

[12] Callaway J 1963 Phys. Rev. 130 A549

Callaway J 1964 Phys. Rev. 134 A998

[13] Tharmalingam K 1963 Phys. Rev. 1302204

[14] Duke C B 1965 Phys. Rev. Lett. 15625

Duke C B and Alferieff M E 1966 Phys. Rev. 145583 
[15] Seraphin B O and Bottka N 1965 Phys. Rev. 139 A560

[16] Aspnes D E 1966 Phys. Rev. 147554

[17] Ralph H I 1968 J. Phys. C: Solid State Phys. 1378

[18] Penchina C M, Pribram J K and Sak J 1969 Phys. Rev. 1881240

[19] Dow J D and Redfield D 1970 Phys. Rev. B 13358

[20] Blossey D F 1970 Phys. Rev. B 23976

[21] Vinogradov V S 1971 Fiz. Tverdogo Tela 133266 (Engl. transl. 1972 Sov. Phys.-Solid State 13 2745) Vinogradov V S 1973 Fiz. Tverdogo Tela 15395 (Engl. transl. 1973 Sov. Phys.-Solid State 15 285)

[22] Arutyunyan I N and Askar'yan G A 1970 Piz. Zh. Eksp. Teor. Fiz. 12378 (Engl. transl. JETP Lett. 12 259)

[23] Nikishov A I 1972 Zh. Eksp. Teor. Fiz. 62562 (Engl. transl. Sov. Phys.-JETP 35 298)

[24] Slonim V Z and Dalidchik F I 1976 Zh. Exp. Teor. Fiz. 712057 (Engl. transl. 1976 Sov. Phys.-JETP 44 1081)

[25] Manakov N L and Fainshtein A G 1980 Zh. Eksp. Teor. Fiz. 79751 (Engl. transl. 1980 Sov. Phys.-JETP 52 382)

[26] Feneuille S, Liberman S, Pinard J and Jacquinot P 1977 C. R. Acad. Sci. B 284291 Liberman S and Pinard J 1979 Phys. Rev. A 20507 Feneuille S, Liberman S, Pinard J and Taleb A 1979 Phys. Rev. Lett. 421404

[27] Freeman R R, Economou N P, Bjorklund G C and Lu K T 1978 Phys. Rev. Lett. 421463 Freeman R R and Economou N P 1979 Phys. Rev. A 202356

[28] Rau A R P 1979 J. Phys. B: At. Mol. Phys. 12 L193 Rau A R P and Lu K T 1980 Phys. Rev. A 211057

[29] Luc-Koenig E and Bachelier A 1979 Phys. Rev. Lett. 43921 Luc-Koenig E and Bachelier A 1980 J. Phys. B: At. Mol. Phys. 131743 Luc-Koenig E and Bachelier A 1980 J. Phys. B: At. Mol. Phys. 131769

[30] Kondratovich V D and Ostrovskii V N 1980 Zh. Eksp. Teor. Fiz. 79395 (Engl. transl. Sov. Phys.-JETP 52 198) Demkov Yu N, Kondratovich V D and Ostrovskii V N 1981 Piz. Zh. Eksp. Teor. Fiz. 34425 (Engl. transl. JETP Lett. 34 403)

Kondratovich V D and Ostrovskii V N 1984 J. Phys. B: At. Mol. Phys. 171981 Kondratovich V D and Ostrovskii V N 1984 J. Phys. B: At. Mol. Phys. 172011

[31] Fabrikant I I 1980 Zh. Eksp. Teor. Fiz. 792070 (Engl. transl. 1980 Sov. Phys.-JETP 52 1045)

[32] Fano U 1981 Phys. Rev. A 24619

[33] Harmin D A 1981 Phys. Rev. A 242491

Harmin D A 1982 Phys. Rev. Lett. 49128 Harmin D A 1982 Phys. Rev. A 262656

[34] Fabrikant I I 1982 Zh. Eksp. Teor. Fiz. 831675 (Engl. transl. 1983 Sov. Phys.-JETP 56 967)

[35] Glab W L, Ng K, Yao D and Nayfeh M H 1985 Phys. Rev. A 313677 Hillard G B and Glab W L 1987 Physica C 145229

[36] Reinhardt W P 1983 J. Phys. B: At. Mol. Phys. 16 L635

Reinhardt W P 1985 Atomic Excitation and Recombination in External Fields ed M H Nayfeh and C W Clark (New York: Gordon and Breach) p 85

[37] Bryant H C et al 1987 Phys. Rev. Lett. 582412

[38] Rau A R P and Wong H Y 1988 Phys. Rev. A 37632

[39] Wong H Y, Rau A R P and Greene C H 1988 Phys. Rev. A 372393

[40] Greene C H and Rouze N 1988 Z. Phys. D 9219

[41] Du M L and Delos J B 1988 Phys. Rev. A 385609

[42] Du M L 1989 Phys. Rev. A 404983

[43] Kondratovich V D and Ostrovsky V N 1990 J. Phys. B: At. Mol. Opt. Phys. 2321 cf section 4

[44] Fabrikant I I 1990 J. Phys. B: At. Mol. Opt. Phys. 231139

[45] Ostrovsky V N and Telnov D A 1991 J. Phys. B: At. Mol. Opt. Phys. 23 L477

Ostrovsky V N and Telnov D A 1993 J. Phys. B: At. Mol. Opt. Phys. 26415

[46] Ostrovsky V N and Telnov D A 1993 Laser Phys. 3495

[47] Nicolaides C A and Mercouris Th 1989 Chem. Phys. Lett. 15945

[48] Fabrikant I I 1989 Phys. Rev. A 402373

[49] Fabrikant I I 1994 J. Phys. B: At. Mol. Opt. Phys. 274545

[50] Gibson N D, Davies B J and Larson D J 1993 Phys. Rev. A 471946

Gibson N D, Davies B J and Larson D J 1993 Phys. Rev. A 48310

[51] Gao B and Starace A F 1990 Phys. Rev. A 425580

[52] Bao M Q, Fabrikant I I and Starace A F 1998 Phys. Rev. A 58411

[53] Slonim V Z and Greene C H 1991 Radiat. Effects Defects Solids 122-3 679 
[54] Du N Y, Fabrikant I I and Starace A F 1993 Phys. Rev. A 482968

[55] Delone N B, Manakov N L and Fainshtein A G 1984 Zh. Eksp. Teor. Fiz. 86906 (Engl. transl. 1984 Sov. Phys.-JETP 59 529)

[56] Madajczyk J L, Pont M, Potvliege R M and Shakeshaft R 1992 Phys. Rev. A 454848

[57] Demkov Yu N, Kondratovich V D and Ostrovsky V N 1981 Piz. Zh. Eksp. Teor. Fiz. 34425 (Engl. transl. 1981 JETP Lett. 34 403)

[58] Blondel C, Delsart C and Dulieu F 1996 Phys. Rev. Lett. 773755

Blondel C, Delsart C and Dulieu F 1999 Phys. Rev. A 593809

[59] Shirley J H 1965 Phys. Rev. 138 B979

[60] Ritus V I 1966 Zh. Eksp. Teor. Fiz. 511544 (Engl. transl. 1967 Sov. Phys.-JETP 25 1027)

[61] Floquet G 1883 Ann. Ecole Norm. Sup. 1247

[62] Zel'dovich Ya B 1973 Usp. Fiz. Nauk 110139 (Engl. transl. 1973 Sov. Phys.-Usp. 16 427)

[63] Sambe H 1973 Phys. Rev. A 72203

[64] Fainshtein A G, Manakov N L and Rapoport L P 1978 J. Phys. B: At. Mol. Phys. 112561

[65] Milfeld K F and Wyatt R E 1983 Phys. Rev. A 2772

[66] Baz A I, Zel'dovich Ya B and Perelomov A M 1971 Scattering, Reactions and Decays in Nonrelativistic Quantum Mechanics 2nd edn (Moscow: Nauka) (in Russian) (note that the 1st edn is available in English translation from the US Department of Commerce, Natural Technical Information Service, no N69 25848)

[67] Malkin I A and Man'ko V I 1979 Dynamic Symmetries and Coherent States of Quantum Systems (Moscow: Nauka) (in Russian)

[68] Manakov N L, Rapoport L P and Fainshtein A G 1977 Teor. Mat. Fiz. 30395 (Engl. transl. 1977 Theor. Math. Phys. 30 255)

[69] Fainshtein A G, Manakov N L and Rapoport L P 1978 J. Phys. B: At. Mol. Phys. 112578

[70] Ostrovsky V N, Kazansky A K and Solov'ev V A 1976 Zh. Eksp. Teor. Fiz. 70493 (Engl. transl. 1976 Sov. Phys.-JETP 43 254)

[71] Stone A D, Azbel M Ya and Lee P A 1985 Phys. Rev. B 311707

[72] Lisitsa V S 1971 Opt. Spektrosk. 31862 (Engl. transl. 1971 Opt. Spectrosc. (USSR) 31 468)

[73] Kovarskii V A and Perel'man N F 1971 Zh. Eksp. Teor. Fiz. 611389 (Engl. transl. 1972 Sov. Phys.-JETP 34 738)

[74] Zon B A and Sholokhov Yu V 1976 Zh. Eksp. Teor. Fiz. 70887 (Engl. transl. 1976 Sov. Phys.-JETP 43 461)

[75] Manakov N L, Ovsiannikov V D and Rapoport L P 1976 Zh. Eksp. Teor. Fiz. 701697 (Engl. transl. 1976 Sov. Phys.-JETP 43 885)

[76] Kazansky A K, Nakamura H and Ostrovsky V N 1997 Laser Phys. 7773

[77] Maquet A 1977 Phys. Rev. A 151088

[78] Zon B A, Manakov N L and Rapoport L P 1975 Opt. Spektrosk. 3713 (Engl. transl. 1975 Opt. Spectrosc. (USSR) 38 6)

Krylovetsky A A, Manakov N L and Marmo S I 1997 Laser Phys. 71756

[79] Bureeva L A and Lisitsa V S 1997 A Perturbed Atom (Moscow: Intra) (in Russian)

[80] Melikyan A O 1975 Zh. Eksp. Teor. Fiz. 681228 (Engl. transl. 1975 Sov. Phys.-JETP 41 410)

Melikyan A O 1977 Kvantovaya Electron. 4429 (Engl. transl. 1977 Sov. J. Quantum Electron. 7 237)

[81] Braun P A and Miroshnichenko G P 1980 Opt. Spektrosk. 491024 (Engl. transl. 1980 Opt. Spectrosc. (USSR) 49561 )

[82] Dion D R and Hirschfelder J O 1976 Adv. Chem. Phys. 35265

[83] Chu S I 1985 Adv. At. Mol. Phys. 21197

[84] Berson I J 1975 J. Phys. B: At. Mol. Phys. 83078

[85] Faisal F H M 1987 Phys. Lett. A 119375

Faisal F H M 1987 Phys. Lett. A 125200

[86] Kamiński J Z 1987 Phys. Lett. A 120396

Kamiński J Z 1995 Phys. Rev. A 524976

[87] Gavrila M 1989 Collision Theory for Atoms and Molecules (NATO ASI Series: Series B vol 196) ed F A Gianturco (New York: Plenum)

Gavrila M 1992 Adv. At. Mol. Opt. Phys. Suppl. 1435

[88] Ehlotzky F, Jaron A and Kamiński J Z 1998 Phys. Rep. 29763

[89] Joachain C J, Dörr M and Kylstra N 2000 Adv. At. Mol. Opt. Phys. 42225

[90] Landau L D and Lifshitz E M 1992 Quantum Mechanics 4th edn (Oxford: Pergamon) section 134

[91] Goldberger M L and Watson K M 1964 Collision Theory (New York: Wiley)

[92] Bohm A, Gadella M and Mainland G B 1989 Am. J. Phys. 571103

[93] Manakov N L and Rapoport L P 1975 Zh. Eksp. Teor. Fiz. 69842 (Engl. transl. 1975 Sov. Phys.-JETP 42 430) 
[94] Zel'dovich Ya B, Manakov N L and Rapoport L P 1975 Usp. Fiz. Nauk 117569 (Engl. transl. 1975 Sov. Phys.-Usp. 18 920)

[95] Bunkin F V and Prokhorov A M 1964 Zh. Eksp. Teor. Fiz. 461090 (Engl. transl. 1965 Sov. Phys.-JETP 19 739)

[96] Salzman W R 1974 Phys. Rev. A 10461

[97] Tip A 1983 J. Phys. A: Math. Gen. 163237

[98] Muller H G, Tip A and van der Wiel M J 1983 J. Phys. B: At. Mol. Phys. 16 L679 Muller H G and Tip A 1984 Phys. Rev. A 303039

[99] Andreev S P, Karnakov B M, Mur V D and Polunin V A 1984 Zh. Eksp. Teor. Fiz. 86866 (Engl. transl. 1984 Sov. Phys.-JETP 59 506)

[100] Krstić P S, Milošević D B and Janev R K 1991 Phys. Rev. A 443089

[101] Faisal F H M and Moloney J V 1981 J. Phys. B: At. Mol. Phys. 143603

[102] Millack T 1990 J. Phys. B: At. Mol. Opt. Phys. 231693

[103] Ostrovsky V N 1977 Teor. Mat. Fiz. 33126 (Engl. transl. 1978 Theor. Math. Phys. 33 923)

[104] Potvliege R M and Shakeshaft R 1988 Phys. Rev. A 386191

[105] Manakov N L, Preobragenskii M A, Rapoport L P and Fainshtein A G 1978 Zh. Eksp. Teor. Fiz. 751243 (Engl. transl. 1978 Sov. Phys.-JETP 48 626)

[106] Manakov N L and Fainshtein A G 1981 Teor. Mat. Fiz. 48375 (Engl. transl. 1982 Teor. Math. Phys. 48 815)

[107] Ostrovsky V N and Telnov D A 1987 J. Phys. B: At. Mol. Phys. 202397 Ostrovsky V N and Telnov D A 1987 J. Phys. B: At. Mol. Phys. 202420

[108] Junker B R 1982 Adv. At. Mol. Phys. 18208 Reinchardt W P 1982 Ann. Rev. Phys. Chem. 33223

[109] Yamani H A and Reinhardt W P 1975 Phys. Rev. A 111144

[110] Chu S I and Reinhardt W P 1977 Phys. Rev. Lett. 391195

[111] Maquet A, Chu S I and Reinhardt W P 1983 Phys. Rev. A 272946

[112] Potvliege R M and Shakeshaft R 1992 Atoms in Intense Laser Fields ed M Gavrila (New York: Academic) p 373

[113] Potvliege R M 1996 Super-Intense Laser-Atom Physics IV (NATO ASI Ser. 3 vol 313) ed H G Muller and M V Fedorov (Dordrecht: Kluwer) p 133

[114] Potvliege R M and Shakeshaft R 1990 Phys. Rev. A 411609

[115] Pont M, Shakeshaft R and Potvliege R M 1990 Phys. Rev. A 426969

[116] Manakov N L and Fainshtein A G 1979 Dokl. Akad. Nauk SSSR 244567 (Engl. transl. 1979 Sov. Phys. Dokl. 24 41)

[117] Alvarez G and Sundaram B 1990 Phys. Rev. A 42452

[118] Graffi S, Grecchi V and Silverstone H G 1985 Ann. Inst. H Poincaré 42215

[119] Latinne O, Kylstra N J, Dörr M, Purvis J, Terao-Duneseath M, Joachain J C, Burke P G and Noble C J 1995 Phys. Rev. Lett. 7446

[120] Becker W, Long S and McIver J K 1990 Phys. Rev. A 424416 Becker W, Long S and McIver J K 1992 Phys. Rev. A 46 R5334 Becker W, Long S and McIver J K 1994 Phys. Rev. A 501540 Lohr A, Becker W and Kleber M 1997 Laser Phys. 7615 Becker W, Lohr A, Kleber M and Lewenstein M 1997 Phys. Rev. A 56645

[121] Kulander K C, Schafer K J and Krause J L 1993 Super-Intense Laser-Atom Physics (NATO ASI Series B: Physics vol 316) ed B Piraux et al (New York: Plenum) p 95 Corkum P B 1993 Phys. Rev. Lett. 711994

[122] Ho T S, Chu S I and Tietz J V 1983 Chem. Phys. Lett. 96464 Ho T S and Chu S I 1984 J. Phys. B: At. Mol. Phys. 172101

[123] Bor H 1947 Almost Periodic Functions (New York: Chelsea)

[124] Fainshtein A G, Manakov N L, Ovsiannikov V D and Rapoport L P 1992 Phys. Rep. 210111

[125] Muller H G and Tip A 1986 J. Phys. A: Math. Gen. 19903

[126] Keldysh L V 1964 Zh. Eksp. Teor. Fiz. 471945 (Engl. transl. 1965 Sov. Phys.-JETP 20 1307)

[127] Protopapas M, Keitel C H and Knight P L 1997 Rep. Prog. Phys. 60389

[128] Saliéres P, L’Huillier A, Antoine P and Lewenstein M 1999 Adv. At. Mol. Opt. Phys. 4183

[129] Dörr M, Potvliege R M, Proulx D and Shakeshaft R 1991 Phys. Rev. A 44574 Dörr M, Potvliege R M, Proulx D and Shakeshaft R 1992 Phys. Rev. A 452142

[130] Potvliege R M and Smith P H G 1991 J. Phys. B: At. Mol. Opt. Phys. 24 L641 Potvliege R M and Smith P H G 1992 J. Phys. B: At. Mol. Opt. Phys. 252501 Potvliege R M and Smith P H G 1994 Phys. Rev. A 493110 
[131] Long S, Becker W and McIver J K 1995 Phys. Rev. A 522262

Eichmann H, Egbert A, Nolte S, Momma C, Wellegehausen B, Becker W, Long S and McIver J K 1995 Phys. Rev. A 51 R3414

Paulus G G, Becker W and Walther H 1995 Phys. Rev. A 524043

Lohr A, Long S, Becker W and McIver J K 1996 Super-Intense Laser-Atom Physics IV (NATO ASI Ser. 3 vol 313) ed H G Muller and M V Fedorov (Dordrecht: Kluwer) p 477

[132] Milošević D B and Piraux B 1996 Phys. Rev. A 541522

[133] Telnov D A, Wang J and Chu S I 1995 Phys. Rev. A 514797

[134] Manakov N L and Fainshtein A G 1984 Zh. Eksp. Teor. Fiz. 871552 (Engl. transl. 1984 Sov. Phys.-JETP 60 890)

[135] Ho T S, Wang K and Chu S I 1986 Phys. Rev. A 331798

[136] Chu S I 1989 Adv. Chem. Phys. 73739

[137] Peskin U and Moiseyev N 1993 J. Chem. Phys. 994590

[138] Yao G and Wyatt R E 1994 J. Chem. Phys. 1011904

[139] Ho T S and Chu S I 1987 Chem. Phys. Lett. 141315

[140] Day H C, Piraux B and Potvlige R M 2000 Phys. Rev. A 61 031402(R)

[141] Barash D, Orel A E and Baer R 2000 Phys. Rev. A 61013402

[142] Rapoport L P,Zon B A and Manakov N L 1978 Theory of Multiphoton Processes in Atoms (Moscow: Atomizdat) (in Russian)

[143] Manakov N L, Rapoport L P and Fainshtein A G 1981 Izv. Akad. Nauk SSSR: Ser. Fiz. 452401

[144] Gavrila M 1997 Photon and Electron Collisions with Atoms and Molecules ed P G Burke and C J Joachain (New York: Plenum) p 147

[145] Moiseyev N 1998 Phys. Rep. 302211

[146] Mercouris T, Themelis S I and Nicolaides C A 2000 Phys. Rev. A 61013407

[147] Messiah A 1966 Quantum Mechanics vol II (New York: Wiley)

[148] Feynman R P 1948 Rev. Mod. Phys. 20367

Feynman R P and Hibbs A R 1965 Quantum Mechanics and Path Integrals (New York: McGraw-Hill)

[149] Kazakov A E, Makarov V P and Fedorov M V 1976 Zh. Eksp. Teor. Fiz. 7038 (Engl. transl. 1976 Sov. Phys.-JETP 43 20)

[150] Landau L D and Lifshitz E M 1975 The Classical Theory of Fields 4th edn (Oxford: Pergamon) section 50

[151] Blum K 1981 Density Matrix Theory and Applications (New York: Plenum) section 1.2.5

[152] Mur V D, Popov V S and Karnakov B M 1999 Zh. Eksp. Teor. Fiz. 115521 (Engl. transl. 1999 Sov. Phys.-JETP 88 286)

[153] Radzig A A and Smirnov B M 1985 Reference Data on Atoms, Molecules and Ions (Berlin: Springer)

[154] Adelman S A 1972 Phys. Rev. A 5508

[155] Demkov Yu N and Drukarev G F 1964 Zh. Eksp. Teor. Fiz. 47918 (Engl. transl. 1965 Sov. Phys.-JETP 20 614)

[156] Abramowitz M and Stegun I A (ed) 1965 Handbook of Mathematical Functions (New York: Dover) section 10.4

[157] Alliluev S P, Weinberg V M and Popov V S 1982 Zh. Eksp. Teor. Fiz. 8277 (Engl. transl. 1982 Sov. Phys.-JETP 55 46)

[158] Krainov V P and Mulykov Z S 1993 Laser Phys. 375

[159] Benassi L, Grecchi V, Harrell E and Simon B 1979 Phys. Rev. Lett. 42704

[160] Kukulin V I, Krasnopol'sky V M and Horacek J 1989 Theory of Resonances (Prague: Academia)

[161] Watson D K 1986 Phys. Rev. A 341016

[162] Rescigno T N and McCurdy C W 1986 Phys. Rev. A 341882

[163] Hokkyo N 1965 Prog. Theor. Phys. 331116

[164] More R M and Gerjuoy E 1973 Phys. Rev. A 71288

[165] Zel'dovich Ya B 1961 Zh. Eksp. Teor. Fiz. 39776 (Engl. transl. 1961 Sov. Phys.-JETP 12 542)

[166] Potvliege R M and Shakeshaft R 1989 Phys. Rev. A 403061

[167] Ben-Tal N, Moiseyev N, Kozloff R and Cerjan Gh 1993 J. Phys. B: At. Mol. Opt. Phys. 261445

[168] Borca B, Flegel A V, Frolov M V, Manakov N L, Milošević D B and Starace A F 2000 Phys. Rev. Lett. 85732

[169] Manakov N L, Marmo S I and Fainshtein A G Zh. Eksp. Teor. Fiz. 9151 (Engl. transl. 1986 Sov. Phys.-JETP $6429)$

[170] Berestetskii V B, Lifshitz E M and Pitaevskii L P 1982 Quantum Electrodynamics 4th edn (Oxford: Pergamon) section 59

[171] Manakov N L and Meremianin A V 1997 Zh. Eksp. Teor. Fiz. 1111984 (Engl. transl. 1997 Sov. Phys.-JETP 84 1080)

[172] Manakov N L 1994 Zh. Eksp. Teor. Fiz. 1061286 (Engl. transl. 1994 Sov. Phys.-JETP 79 696)

[173] Manakov N L and Ovsiannikov V D 1997 J. Phys. B: At. Mol. Opt. Phys. 302109 
[174] Manakov N L, Ovsiannikov V D and Starace A F 1999 Phys. Rev. Lett. 824791

[175] Manakov N L 1966 Super-Intense Laser-Atom Physics IV (NATO ASI Ser. 3 vol 313) ed H G Muller and M V Fedorov (Dordrecht: Kluwer) p 153

[176] Smirnov B M and Chibisov M I 1965 Zh. Eksp. Teor. Fiz. 49841 (Engl. transl. 1966 Sov. Phys.-JETP 22 585)

[177] Bethe H A and Peierls R E 1935 Proc. R. Soc. A 148146

[178] Armstrong B H 1963 Phys. Rev. 1311132

[179] Borca B, Frolov M V, Manakov N L and Starace A F to be published

[180] Peierls R 1979 Surprises in Theoretical Physics (Princeton, NJ: Princeton University Press) section 1.1

[181] Dalidchik F I and Slonim V Z 1976 Zh. Eksp. Teor. Fiz. 7047 (Engl. transl. 1976 Sov. Phys.-JETP 43 25) 